\title{
SPINOR CONDENSATES AND LIGHT SCATTERING FROM BOSE-EINSTEIN CONDENSATES
}

\author{
WOLFGANG KETTERLE \\ Department of Physics and Research Laboratory of \\ Electronics, Massachusetts Institute of Technology, \\ Cambridge, MA 02139, USA \\ E-mail: ketterle@mit.edu
}

PHOTO: height $7.5 \mathrm{~cm}$, width $11 \mathrm{~cm}$ 


\section{Contents}

\begin{tabular}{ll|l}
\hline Introduction & 3
\end{tabular}

2 Optical properties of a Bose-Einstein condensate $\quad 4$

2.1 Light scattering from a Bose-Einstein condensate . . . . . . . . . . . . . . . . . . . . . . . . 4

2.2 The dynamic structure factor of a Bose-Einstein condensate . . . . . . . . . . . . . . . . 8

2.3 Experimental aspects of Bragg spectroscopy . . . . . . . . . . . . . . . . . . . . . . . . 14

2.4 Light scattering in the free-particle regime . . . . . . . . . . . . . . . . . . . . . . . . . 15

2.5 Light scattering in the phonon regime . . . . . . . . . . . . . . . . . . . . . . . . . . 20

3 Amplified scattering of light $\quad 22$

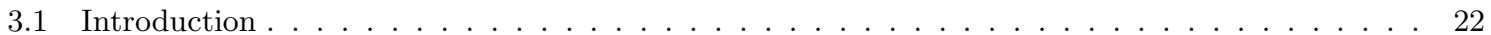

3.2 Superradiant Rayleigh scattering _ . . . . . . . . . . . . . . . . . . . . . . . . . 23

3.3 Phase-coherent amplification of matter waves . . . . . . . . . . . . . . . . . . . . . . . . . . . 31

4 Spinor Bose-Einstein condensates 33

4.1 The implications of rotational symmetry . . . . . . . . . . . . . . . . . . . . . . . . . 34

4.2 Tailoring the ground-state structure with magnetic fields . . . . . . . . . . . . . . . . . 37

4.3 Spin-domain diagrams: a local density approximation to the spin structure of spinor condensates 39

4.4 Experimental methods for the study of spinor condensates . . . . . . . . . . . . . . . . . 40

4.5 The formation of ground-state spin domains . . . . . . . . . . . . . . . . . . . . . . . . 40

4.6 Miscibility and immiscibility of spinor condensate components . . . . . . . . . . . . . . . . . . . 43

$4.7 \quad$ Metastable states of spinor Bose-Einstein condensates . . . . . . . . . . . . . . . . . . . . . . . 44

4.8 Quantum tunneling . . . . . . . . . . . . . . . . . . . . . . . . . . . 47

4.9 Magnetic field dependence of spin-domain boundaries . . . . . . . . . . . . . . . . . . . . . . 52 


\title{
SPINOR CONDENSATES AND LIGHT SCATTERING FROM BOSE-EINSTEIN CONDENSATES
}

\author{
Dan M. Stamper-Kurn ${ }^{1}$, Wolfgang Ketterle ${ }^{2}$
}

\begin{abstract}
These notes discuss two aspects of the physics of atomic Bose-Einstein condensates: optical properties and spinor condensates. The first topic includes light scattering experiments which probe the excitations of a condensate in both the free-particle and phonon regime. At higher light intensity, a new form of superradiance and phase-coherent matter wave amplification were observed. We also discuss properties of spinor condensates and describe studies of ground-state spin domain structures and dynamical studies which revealed metastable excited states and quantum tunneling.
\end{abstract}

\section{Introduction}

The possibility of creating optical fields with many photons in a single mode of a resonator was realized with the creation of the laser in 1960 . The possibility of creating a matter-wave field with many atoms in a single mode of an atom trap (which is the atomic equivalent of an optical resonator) was realized with the achievement of Bose-Einstein condensation (BEC) in 1995. Because of the wealth of new phenomena which the condensates display, and the precision and flexibility with which they can be manipulated, interest in them has grown explosively in the communities of atomic physics, quantum optics, and many-body physics. At least twenty groups have created condensates, and the publication rate on Bose-Einstein condensation has soared following the discovery of the gaseous condensates.

Although atomic condensates and laser light share many properties, they also differ fundamentally: atoms interact readily, while photons do not. As a result, the atomic condensates constitute a novel class of many-body systems that provide a new laboratory for many-body physics. They have already yielded discoveries such as stability and collapse of condensates with attractive interactions, multicomponent condensates, Feshbach resonances and novel optical properties, and have led to advances in many-body theory. Furthermore, because atoms interact, atom optics is inherently non-linear optics. Consequently, nonlinear effects such as four-wave mixing that were first achieved with light only with difficulty, occur almost automatically with coherent matter waves.

These lecture notes will focus on two aspects of Bose-Einstein condensation: light scattering from a Bose-Einstein condensate and spinor condensates. Our lectures at Les Houches covered a broader range of topics, including trapping techniques, methods to probe the condensate and studies of sound and condensate formation. For those topics we refer to our Varenna Summer School Notes which give a comprehensive discussion of experimental techniques, static and dynamic properties, coherence and optical trapping of condensates [1]. This paper and some other recent review papers summarize the state of the field 2 , 何.

Research on gaseous BEC can be divided into two areas: In the first, which could be labeled "the atomic condensate as a coherent gas" or "atom lasers," one would like to have as little interaction as possible between atoms - almost like photons in an optical laser. Thus the experiments are

\footnotetext{
${ }^{1}$ Norman Bridge Laboratory of Physics, California Institute of Technology, Pasadena, CA 91125, USA

${ }^{2}$ Department of Physics and Research Laboratory of Electronics, Massachusetts Institute of Technology, Cambridge, MA 02139, USA
}

(C) EDP Sciences, Springer-Verlag 1999 
preferentially done at low densities. The Bose-Einstein condensate serves as an intense source of ultracold coherent atoms for experiments in atom optics, in precision studies or for explorations of basic aspects of quantum mechanics. The second area could be labeled as "BEC as a new quantum fluid" or "BEC as a many-body system." The focus here is on the interactions between the atoms which are most pronounced at high densities.

The topics covered in these notes illustrate both aspects of BEC. Spinor condensates realize a new class of quantum fluids. Coherent matter wave amplification is at the heart of atom lasers. Our studies of light scattering from a Bose condensate link both aspects together: light scattering was used to imprint phonons into the condensate, but also to measure the coherence of an atom laser and to realize a matter wave amplifier.

Chapters 2 and 4 of this review are based on the thesis of one of the authors [5]. An abbreviated version of chapter 2 will appear in Ref. [6].

\section{Optical properties of a Bose-Einstein condensate}

What does a trapped Bose-Einstein condensate look like? More precisely, how does it interact with light, and does this differ fundamentally from what one would naively expect from a similar collection of very cold atoms? In the early 1990s, before Bose-Einstein condensation was realized in atomic gases, there were lively debates about how a condensate could be observed. Some researchers thought it would absorb all light and would therefore be "pitch black," some predicted it would be "transparent" (due to superradiant line-broadening [7]), others predicted that it would reflect light due to polaritons [8, 9] and be "shiny" like a mirror.

All the observations of Bose condensates have employed scattering or absorption of laser light. These observations were either done on ballistically expanding dilute clouds or with far-off-resonant light. Under those circumstances, a Bose condensate scatters light as ordinary atoms do. On resonance, the condensate strongly absorbs the light, giving rise to the well-known "shadow pictures" of expanding condensates where the condensate appears black. For off-resonant light, the absorption can be made negligibly small, and the condensate acts as a dispersive medium bending the light like a glass sphere. This regime has been used for non-destructive in-situ imaging of Bose-Einstein condensates.

Our group has recently looked more closely at how coherent, weakly-interacting atoms interact with coherent light. Light scattering imparts momentum to the condensate and creates an excitation in a many-body system (Fig. 11). Consequently, the collective nature of excitations and the coherence of the condensate can affect its optical properties. Thus light scattering can be used to illuminate properties of the condensate.

\subsection{Light scattering from a Bose-Einstein condensate}

\subsubsection{Elastic and inelastic light scattering}

Let us begin by considering the effect on a single atom of a single light scattering event. The initial state of the atom-light system is $\left|N_{k}, \ldots 0_{l} \ldots ; i\right\rangle$ where $N_{k}$ photons are in an incident beam with wavevector $k$, no photons are in other photon modes (such as mode $l$ ), and the atom is in state $|i\rangle$ which can be either a trapped or untrapped state. After adiabatically eliminating the excited atomic state, the coupling between the atom and the light is described by the operator

$$
\mathcal{H}^{\prime}=C \sum_{k, l, m, n} \hat{c}_{l}^{\dagger} \hat{a}_{n}^{\dagger} \hat{c}_{k} \hat{a}_{m} \delta_{l+n-k-m}
$$

Here $\hat{c}_{k}\left(\hat{c}_{k}^{\dagger}\right)$ is the destruction (creation) operator for optical waves, and $\hat{a}_{k}\left(\hat{a}_{k}^{\dagger}\right)$ is the destruction (creation) operator for atomic waves of wavevector $k$. The strength of atomic resonances and the detuning of the light determines the strength of the coupling, summarized in the coefficient $C$.

Due to the coupling, light is scattered from the incident beam to wavevector $l=k-q$ with a 


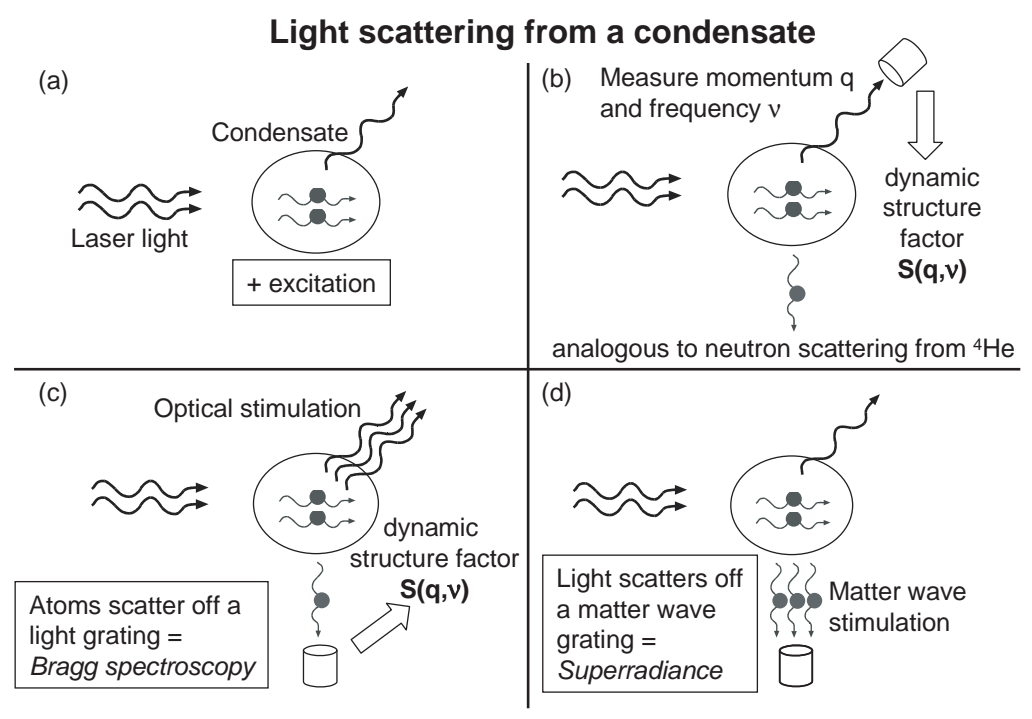

Fig. 1. Light scattering from a Bose-Einstein condensate. When a photon is scattered, it transfers momentum to the condensate and creates an excitation (upper left). Therefore, an analysis of the scattered light allows the determination of the dynamic structure factor, in close analogy to neutron scattering experiments with superfluid helium (upper right). For sufficiently large momentum transfer the excitation leads to an atom scattered out of the condensate. The signal is greatly increased by stimulating the light scattering by a second laser beam and detecting the scattered atoms (lower left) — this is the scheme for Bragg spectroscopy. Light scattering can also be stimulated by adding a coherent atomic field (lower right). This led to superradiant scattering of light and atoms. 
cross-section proportional to円

$$
\begin{aligned}
\frac{d \sigma}{d \Omega} \propto & |C|^{2} \times \\
& \left(\left|\left\langle N_{k}-1, \ldots 1_{l} \ldots ; i\left|\sum_{m} \hat{c}_{k-q}^{\dagger} \hat{a}_{m+q}^{\dagger} \hat{c}_{k} \hat{a}_{m}\right| N_{k}, \ldots 0_{l} \ldots ; i\right\rangle\right|^{2}\right. \\
& \left.+\sum_{j \neq i}\left|\left\langle N_{k}-1, \ldots 1_{l} \ldots ; j\left|\sum_{m} \hat{c}_{k-q}^{\dagger} \hat{a}_{m+q}^{\dagger} \hat{c}_{k} \hat{a}_{m}\right| N_{k}, \ldots 0_{l} \ldots ; i\right\rangle\right|^{2}\right)
\end{aligned}
$$

Two contributions to the light scattering are separated in the above expression. The first part describes "coherent" light scattering, i.e. diffraction and refraction, in which the atomic system is left in its original state, and the scattered light has the same frequency as the incident light. The coupling shifts the phase of the elastically scattered light by an amount proportional to $\left\langle N_{k}-\right.$ $\left.1, \ldots 1_{l} \ldots ; i\left|\mathcal{H}^{\prime}\right| N_{k}, \ldots 0_{l} \ldots ; i\right\rangle$. This phase shift can be used to spatially image an atomic cloud by a dispersive imaging technique, such as dark-ground or phase-contrast imaging. For a dilute cloud of size $d$, the coherent scattering is limited to the diffraction angle $\lambda / d$. When the cloud is much larger than an optical wavelength $\lambda$, only small-angle forward scattering is coherent.

The second part of the scattering cross-section describes "incoherent" light scattering in which the state of the atom is changed. For the case considered here, $d \gg \lambda$, this occurs when light is scattered outside the diffraction angle. Incoherent, or inelastic light scattering is used for absorption imaging where the light which is transmitted by the cloud is collected, and the amount of probe light scattered out of the imaging system is determined.

One can learn more from inelastic scattering than just by observing absorption, i.e. counting the total sum of scattered photons. An inelastically scattered photon is shifted in frequency from the incident photon. Further, the outgoing angle of the scattered photon determines the momentum $\hbar \vec{q}$ which is imparted to the sample. Thus, a spectroscopic analysis of inelastically scattered photons at a given angle from the incident light beam determines the response of the atomic sample to a given energy and momentum transfer (Fig. 1).

Analyzing photons scattered by a Bose-Einstein condensate from a single beam would be a difficult task. Alkali Bose-Einstein condensates are currently produced with $\lesssim 10^{7}$ atoms. If one would scatter light from a small fraction of these over a $4 \pi$ solid angle, only a few photons would be collected and one would need to determine their frequency amidst a large background of incident and scattered light.

Instead, we have adopted a different approach. Rather than detecting spontaneous scattering from a single beam, we study light scattering as a stimulated process, called Bragg scattering, induced by two laser beams which illuminate the atomic sample (Fig. 1). The momentum and energy transfer is pre-determined by the angle and frequency difference between the incident beams, respectively, rather than post-determined by the position of a photo-detector and by a difficult frequency measurement. The quantities of interest are matrix elements which characterize the response of the condensate, and they are the same for spontaneous and stimulated scattering. Furthermore, since the momentum transfer can be much greater than the momentum spread of the sub-recoil atomic sample, and since stimulated light scattering can be made to dominate over spontaneous scattering, the response of the system can be assessed by the nearly background-free detection of recoiling atoms.

We have studied Bose-Einstein condensates by the spectroscopic measurement of the Bragg scattering resonance. In this paper, we describe two applications of Bragg spectroscopy to study excitations of a Bose-Einstein condensate in either the free-particle [10] (large momentum transfer) or the phonon [11] (small momentum transfer) regime. The discussion includes a description of the dynamic structure factor of a Bose-Einstein condensate which leads to the interpretation of our measurements

\footnotetext{
${ }^{1}$ Our discussion is limited to low intensity of the probe light, allowing us to neglect the Mollow triplet fluorescence spectrum observed at high intensities.
} 
as an observation of the zero-point momentum distribution of trapped condensates, as a measurement of the energies of free-particle and phonon excitations, and as evidence for correlations in the many-body condensate wavefunction introduced by interatomic interactions.

\subsubsection{Light scattering from atomic beams and atoms at rest}

The interaction of a neutral atomic beam with an optical standing wave was studied by several groups in the early 1980's. Quantitative studies which focused on the effect of conservative optical potentials were performed by Pritchard and collaborators [12, 13]. In two different experiments, a collimated atomic beam of sodium was incident upon a standing light wave formed by a retro-reflected laser beam. Two regimes of scattering were identified: Kapitza-Dirac scattering from a thin optical grating (tightly focused beams) which is non-specific in the angle between the incident atomic beam and the standing wave [12], and Bragg scattering from a thick grating (loosely focused beams) which occurs only at specific resonant angles [13]. In these atomic beam experiments, the kinetic energy of the atoms, and thus the magnitude of their momentum, is unchanged by scattering off the stationary optical field. Thus, scattering can occur only if the optical field contains photons propagating in a direction so that the atomic momentum can be rotated by the absorption and stimulated emission of photons from the standing wave. A thin optical grating contains photons propagating over a wide angular range, and thus scattering is not limited to specific angles. In the case of a broad focus, the angular divergence of the photons is too small to allow scattering except at specific incident angles of the atomic beam to the standing wave (the so-called Bragg angles).

Kapitza-Dirac [14] and Bragg [15] scattering of Bose-Einstein condensates have also been demonstrated. These experiments were performed by exposing the nearly stationary atomic sources to a pulse of two intersecting laser beams which had a variable differential detuning $\omega$. Such an experimental situation is identical to the aforementioned atomic beam experiments when viewed in the frame of reference of the atoms: the duration of the optical pulse corresponds to the width of the optical grating, and a differential detuning between the optical beams is equivalent to an atom crossing an optical grating at an angle which introduces opposite Doppler shifts to the two counter-propagating laser beams. Kapitza-Dirac scattering occurs for short pulses which contain frequency components necessary to excite the atom to an energy of $\hbar \omega_{q}^{0}=\hbar^{2} q^{2} / 2 m$ where $\hbar q$ is the momentum recoil due to a single scattering event. Similarly, the condition for Bragg scattering becomes a resonance condition for exciting an atom to an excited momentum state: $\omega=\omega_{q}^{0}$ (for first order scattering).

The Bragg resonance condition is sensitive to the motion of the atom with respect to the optical standing wave orientation. By simple energy and momentum conservation, the energy transferred to an atom with initial velocity $\vec{v}_{i}$ by a momentum kick of $\hbar \vec{q}$ is

$$
\hbar \omega=\frac{\left(\hbar \vec{q}+m \vec{v}_{i}\right)^{2}}{2 m}-\frac{m v_{i}^{2}}{2}=\frac{\hbar^{2} q^{2}}{2 m}+\hbar \vec{q} \cdot \vec{v}_{i}
$$

Thus, the Bragg resonance is Doppler sensitive and can be used to determine spectroscopically the velocity distribution of an atomic sample. It has been used previously to determine the temperature of laser-cooled atoms [16]. Here we extend the method to the determination of the zero-point motion of a condensate.

\subsubsection{Relation to the dynamic structure factor of a many-body system}

Inelastic scattering has long been used to probe the properties of condensed-matter systems. In the case of liquid helium, both neutron and light scattering were used to determine the elementary excitations of this system [17 21]. A theoretical discussion of the spectrum of inelastically scattered light from a Bose-Einstein condensate has been presented by a number of authors [22 24]. The dilute atomic condensates are particularly simple examples for the general scattering theory since the scattering can be treated in an atomic basis. Following the experimental studies of inelastic light scattering which are summarized in this review, a thorough interpretation of light scattering from an inhomogeneous Bose-Einstein condensate was presented [25]. 
Let us discuss how Bragg scattering is used to probe the properties of a many-body system. An atomic sample is exposed to two laser beams, with wavevectors $\vec{k}_{1}$ and $\vec{k}_{2}$ and a frequency difference $\omega$ which is generally much smaller than the detuning $\Delta$ of the beams from an atomic resonance. The two laser beams interfere to form a "walking" wave intensity modulation $I_{\bmod }(\vec{r}, t)=I \cos (\vec{q} \cdot \vec{r}-\omega t)$ where $\vec{q}=\vec{k}_{1}-\vec{k}_{2}$. Due to the ac Stark effect [26], atoms exposed to this intensity modulation experience a conservative optical potential with a spatial modulation of $V_{\bmod }=\left(\hbar \Gamma^{2} / 8 \Delta\right) \cdot\left(I_{\bmod } / I_{\text {sat }}\right)$ where $\Gamma$ is the line width of the atomic resonance and $I_{\text {sat }}$ the saturation intensity.

To determine the Bragg scattering response of a many-body system, we express the modulated potential in second quantized notation as

$$
V_{\bmod }=\frac{V}{2}\left(\hat{\rho}^{\dagger}(\vec{q}) e^{-i \omega t}+\hat{\rho}^{\dagger}(-\vec{q}) e^{+i \omega t}\right)
$$

where $\hat{\rho}(\vec{q})=\sum_{m} \hat{a}_{m+q}^{\dagger} \hat{a}_{m}$ is the Fourier transform of the atomic density operator at wavevector $\vec{q}$. Equivalently, $V_{\text {mod }}$ is found by isolating those terms in $\mathcal{H}^{\prime}$ (Eq. 2.1) which involve the macroscopically occupied optical modes at wavevectors $\vec{k}_{1}$ and $\vec{k}_{2}$, and replacing the photon creation and destruction operators with $c$-numbers proportional to the electric field strength of the Bragg scattering laser beams.

We may then determine the Bragg scattering rate using Fermi's golden rule. Considering scattering out of the many-body ground state $|g\rangle$, we neglect the counter-rotating term in $V_{\text {mod }}$ and obtain the excitation rate per particle as

$$
\frac{W}{N}=\frac{2 \pi}{N \hbar}\left(\frac{V}{2}\right)^{2} \sum_{f}\left|\left\langle f\left|\hat{\rho}^{\dagger}(\vec{q})\right| g\right\rangle\right|^{2} \delta\left(\hbar \omega-\left(E_{f}-E_{g}\right)\right) \equiv 2 \pi \omega_{R}^{2} S(\vec{q}, \omega)
$$

Here $N$ is the number of atoms in the system, and the sum is performed over all final excited states $|f\rangle$ with energy $E_{f}$. We have introduced the dynamic structure factor $S(\vec{q}, \omega)$ which is the Fourier transform of density-density fluctuations in state $|g\rangle$ with spatial and temporal frequencies of $\vec{q}$ and $\omega$, respectively [20,21]. The dynamic structure factor generally characterizes the response of the system to longitudinal perturbations of any source, not solely to optical excitation. The density fluctuation spectrum is directly determined by the Bragg scattering response, normalized by the two-photon Rabi frequency $\omega_{R}=V / 2 \hbar$. Integrating over all frequencies $\omega$ one obtains the static structure factor $S(\vec{q})=\left\langle g\left|\hat{\rho}(\vec{q}) \hat{\rho}^{\dagger}(\vec{q})\right| g\right\rangle$ which is equivalent to the line strength of the Bragg resonance.

\subsection{The dynamic structure factor of a Bose-Einstein condensate}

In this section, we use the theory of the weakly-interacting Bose-Einstein condensate to predict the dynamic structure factor, first for a homogeneous condensate and then for the situation of experimental relevance, an inhomogeneous condensate confined by a harmonic trapping potential.

\subsubsection{The homogeneous condensate}

A Bose-Einstein condensate is quite different from other fluids in that the microscopic (i.e. singleatom) excitations of the system become manifest as macroscopic density fluctuations due to interference with the macroscopic wavefunction. Considering density fluctuations in a homogeneous BoseEinstein condensate, we may approximate

$$
|e\rangle=\frac{1}{\sqrt{N}} \hat{\rho}^{\dagger}(\vec{q})|g\rangle=\frac{1}{\sqrt{N}} \sum_{m} \hat{a}_{m+q}^{\dagger} \hat{a}_{m}|g\rangle \simeq \frac{\left(\hat{a}_{q}^{\dagger} \hat{a}_{0}+\hat{a}_{0}^{\dagger} \hat{a}_{-q}\right)|g\rangle}{\sqrt{N}}=\left|e^{+}\right\rangle+\left|e^{-}\right\rangle
$$

Here, the macroscopic occupation of the zero-momentum state picks out two terms in the sum. Following Bogoliubov [27], we identify $\hat{a}_{0}^{\dagger}=\hat{a}_{0}=\sqrt{N_{0}}$ and transform to Bogoliubov operators by substituting $\hat{a}_{k}=u_{k} \hat{b}_{k}-v_{k} \hat{b}_{-k}^{\dagger}$. The operators $\hat{b}_{k}^{\dagger}$ and $\hat{b}_{k}$ are creation and destruction operators for the proper microscopic quasi-particle excitations of the condensate, with $u_{k}=\cosh \phi_{k}, v_{k}=\sinh \phi_{k}$ and 


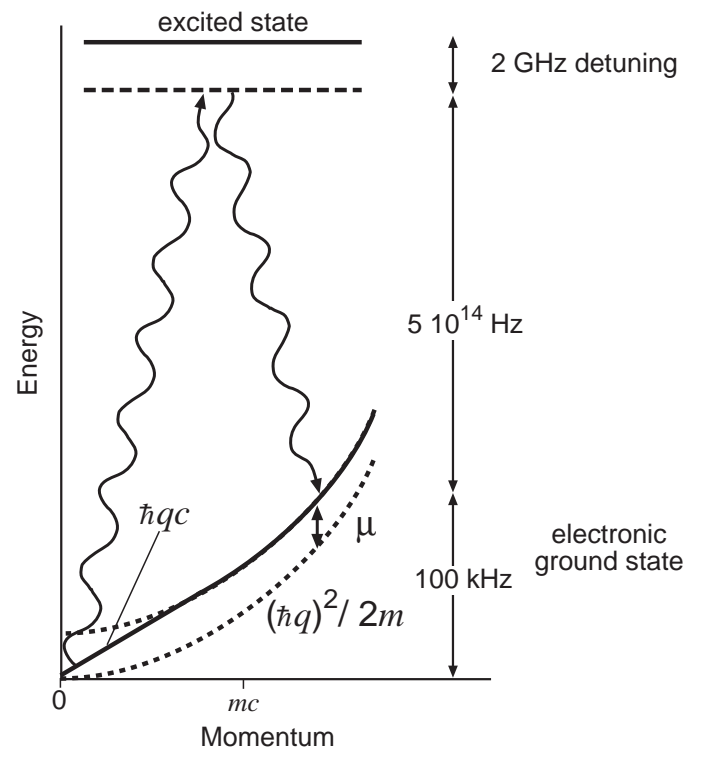

Fig. 2. Probing the dispersion relation of a Bose-Einstein condensate by off-resonant light scattering. The microscopic excitation spectrum in a homogeneous weakly-interacting Bose-Einstein condensate is given by the Bogoliubov dispersion relation (solid line). For small momenta $\hbar q$, such that $\hbar q \ll m c$ the dispersion relation is phonon-like (linear). Here $m$ is the mass, and the speed of sound $c$ is related to the interaction energy $\mu$ by $\mu=m c^{2}$. For large momenta $(\hbar q \gg m c)$ it is particle-like (quadratic) offset from the energy of a free-particle excitation by a mean-field shift of $\mu(\approx 5 \mathrm{kHz}$ for our experiments). Excitations can be created optically by stimulated light scattering using two laser beams which are both far detuned (about $2 \mathrm{GHz}$ ) from the atomic resonance. Momentum and energy are provided by absorption of one photon to a virtual excited level, followed by stimulated emission of a second, lower energy photon.

$\tanh 2 \phi_{k}=\mu /\left(\hbar \omega_{k}^{0}+\mu\right)$. Here, $\mu$ is the chemical potential, and, again, $\hbar \omega_{k}^{0}=\hbar^{2} k^{2} / 2 m$ is the free recoil energy at wavevector $\vec{k}$. The Bogoliubov quasi-particle spectrum is given as $\hbar \omega_{k}^{B}=\sqrt{\hbar \omega_{k}^{0}\left(\hbar \omega_{k}^{0}+2 \mu\right)}$ (see Fig. 2).

The many-body wavefunction of the condensate $|g\rangle$ corresponds to the quasi-particle vacuum defined by the relations $\hat{b}_{k}|g\rangle=0, \forall k$. Thus, we find

$$
\hat{\rho}^{\dagger}(\vec{q})|g\rangle \simeq \sqrt{N_{0}}\left(u_{q}-v_{q}\right) \hat{b}_{q}^{\dagger}|g\rangle
$$

From this, it follows that for a homogeneous Bose-Einstein condensate $S(\vec{q}, \omega) \simeq\left(u_{q}-v_{q}\right)^{2} \delta\left(\omega-\omega_{q}^{B}\right)$, and $S(\vec{q})=\left(u_{q}-v_{q}\right)^{2}=\omega_{q}^{0} / \omega_{q}^{B}$.

Thus, we expect Bragg scattering from an interacting, homogeneous Bose-Einstein condensate to differ from the Bragg scattering of a non-interacting condensate in two ways. First, the Bragg resonance occurs at the Bogoliubov quasi-particle energy which is higher than the free-particle recoil energy, i.e. the Bragg resonance line at a momentum transfer of $\hbar \vec{q}$ is shifted upwards in frequency from the free particle resonance by $\Delta \omega=\omega_{q}^{B}-\omega_{q}^{0}$. Second, the Bragg scattering response is weakened relative to that of free particles by a factor $S(\vec{q})=\left(u_{q}-v_{q}\right)^{2}<1$. In other words, light scattering from a Bose-Einstein condensate is suppressed by the presence of repulsive interatomic interactions. Indeed, these two statements are equivalent: by the $f$-sum rule which states that $\int \omega S(\vec{q}, \omega) d \omega=\omega_{q}^{0}$, an increase in the resonance frequency implies a decrease in the excitation strength [20].

Let us evaluate the structure factor in two limits of the Bragg scattering wavevector $\vec{q}$. The wavevector which corresponds to the interaction energy is the inverse healing length $\xi^{-1}=\sqrt{2} m c / \hbar$ where $c$ is the speed of Bogoliubov sound. For large scattering wavevectors $\left(q \gg \xi^{-1}\right)$, Bragg scattering occurs in the free-particle regime. The Bragg resonance is shifted upwards by the chemical potential 
$\Delta \omega \simeq \mu / \hbar$, and the line strength tends to $S(\vec{q}) \rightarrow 1-\mu / \hbar \omega_{q}^{0}$. Thus, by measuring the frequency shift of the Bragg scattering resonance in the free-particle regime, one can directly measure the chemical potential [10]. For small wavevectors $\left(q \ll \xi^{-1}\right)$, the Bose-Einstein condensate responds to optical excitation collectively with the creation of phonons. The static structure factor tends to $S(\vec{q}) \rightarrow \hbar q / 2 m c$ and vanishes in the long wavelength limit, as required of a zero-temperature system with finite compressibility [28].

\subsubsection{Bragg scattering as a probe of pair correlations in the condensate}

It is interesting to re-examine these modifications to light scattering from a Bose-Einstein condensate in terms of the structure of the many-body condensate wavefunction. What is it about a dilute, weakly-interacting Bose-Einstein condensate that suppresses light scattering compared to noninteracting atoms? As discussed above, the static structure factor $S(\vec{q})$ is the magnitude of the state vector $|e\rangle \simeq\left|e^{+}\right\rangle+\left|e^{-}\right\rangle$(Eq. 2.6 $)$. The states $\left|e^{+}\right\rangle$and $\left|e^{-}\right\rangle$represent two means by which momentum is imparted to the condensate: either by promoting a zero-momentum particle to momentum $\hbar \vec{q}$, or else by demoting a particle from momentum $-\hbar \vec{q}$ to zero momentum.

If correlations could be neglected, the total rate of excitation would simply be the sum of the independent rates for these two processes, proportional to $\left\langle e^{+} \mid e^{+}\right\rangle=\left\langle N_{q}^{0}\right\rangle+1=u_{q}^{2}$ and $\left\langle e^{-} \mid e^{-}\right\rangle=$ $\left\langle N_{-q}^{0}\right\rangle=v_{q}^{2}$ where $\left\langle N_{k}^{0}\right\rangle$ is the expected number of atoms of momentum $\hbar \vec{k}$ in the condensate. Indeed, a simple--minded rate equation leads to an expression for the total scattering rate which is proportional to $N_{0}\left(\left\langle N_{q}^{0}\right\rangle+1\right)+\left\langle N_{-q}^{0}\right\rangle\left(N_{0}+1\right)$. This expression is the sum of the rates of the two processes which can transfer momentum $\hbar \vec{q}$ to the system and scatter particles into or out of the condensate. Each rate is proportional to the number of particles in the initial state and, because of bosonic stimulation, to the number of atoms in the final state plus one. This would apply, for example, to a condensate in a pure number state, or to an ideal gas condensate with a thermal admixture of atoms with momenta $\pm \hbar \vec{q}$, and would always lead to $S(\vec{q})>1$.

Yet, for the many-body ground state of the interacting Bose gas, the behavior is dramatically different. Interactions between zero-momentum atoms admix into the condensate pairs of atoms at momenta $\pm \hbar \vec{q}$ the population of which comprises the quantum depletion [29]. As a result, the two momentum transfer mechanisms described above produce indistinguishable final states, and the total rate of momentum transfer is given by the interference of two amplitudes, not by the sum of two rates. Pair excitations in the condensate are correlated so as to minimize the total energy, and this results in a relative phase between the two amplitudes which produces destructive interference: $S(\vec{q})=\left(u_{q}-v_{q}\right)^{2}<1$. For high momentum, $\left\langle N_{q}^{0}\right\rangle \ll 1$ and the interference plays a minor role. In the phonon regime, while the independent rates $u_{q}^{2}$ and $v_{q}^{2}$ (and hence $\left\langle N_{ \pm q}^{0}\right\rangle$ ) diverge as $1 / q$, the correlated quantum depletion extinguishes the rate of Bragg excitation.

It is interesting to note that the extinction of Bragg scattering at low momentum, i.e. the minus sign in the amplitude $u_{q}-v_{q}$ of state $|e\rangle$, arises from minimizing the energy of an interacting Bose gas with positive scattering length. One might wonder whether a negative sign of the scattering length would lead to an enhancement of scattering. However, homogeneous condensates with negative scattering length are unstable. In a trap, they are only stable when their size is smaller than the healing length, effectively cutting off the long-wavelength phonon modes. Therefore, a condensate with negative scattering length should show essentially the same behavior as a similar-sized condensate of non-interacting atoms.

\subsubsection{Mean-field theory determination of $S(\vec{q}, \omega)$}

These same results are obtained also in a self-consistent mean-field approach. Considering again the homogeneous case, we introduce the perturbation

$$
V_{\bmod }=\frac{V}{2}\left(e^{i \vec{q} \cdot \vec{r}-i \omega t}+e^{-i \vec{q} \cdot \vec{r}+i \omega t}\right)
$$


to the time-dependent Gross-Pitaevskii equation [30,31], and use a condensate wavefunction of the form

$$
\psi(\vec{r}, t)=e^{-i \mu t / \hbar}\left(\psi_{0}(\vec{r}, t)+u(t) e^{i \vec{q} \cdot \vec{r}-i \omega t}+v^{*}(t) e^{-i \vec{q} \cdot \vec{r}+i \omega t}\right)
$$

Here, $\mu=n g$ is the chemical potential where $n$ is the condensate density, $g=4 \pi \hbar^{2} a / m$ and $a$ is the $s^{-}$ wave scattering length. In the absence of the perturbation, the ground-state condensate wavefunction is $\psi_{0}(\vec{r}, t)=\sqrt{n}$.

The weak potential $V_{\text {mod }}$ introduces the small perturbations $u(t) e^{i \vec{q} \cdot \vec{r}-i \omega t}$ and $v(t) e^{-i \vec{q} \cdot \vec{r}+i \omega t}$ where the amplitudes $u(t)$ and $v(t)$ are slowly varying functions of time. Using the Gross-Pitaevskii equation and isolating terms proportional to $e^{i \vec{q} \cdot \vec{r}-i \omega t}$ and $e^{-i \vec{q} \cdot \vec{r}+i \omega t}$, we obtain the set of equations

$$
\begin{aligned}
\hbar \omega u+i \hbar \frac{d u}{d t} & =\left(\frac{\hbar^{2} q^{2}}{2 m}+n g\right) u+n g v+\frac{V}{2} \psi_{0} \\
-\hbar \omega v-i \hbar \frac{d v}{d t} & =\left(\frac{\hbar^{2} q^{2}}{2 m}+n g\right) v+n g u+\frac{V}{2} \psi_{0}
\end{aligned}
$$

Let us now apply the Bogoliubov transformation, and write

$$
\left(\begin{array}{l}
u(t) \\
v(t)
\end{array}\right)=\alpha(t)\left(\begin{array}{c}
u_{q} \\
-v_{q}
\end{array}\right)+\beta(t)\left(\begin{array}{c}
v_{q} \\
-u_{q}
\end{array}\right)
$$

The two component vectors $\left(\begin{array}{c}u_{q} \\ -v_{q}\end{array}\right)$ and $\left(\begin{array}{c}v_{q} \\ -u_{q}\end{array}\right)$ are solutions of the equations 27, 31]

$$
\left[\begin{array}{cc}
\left(\hbar \omega_{q}^{0}+\mu\right) & \mu \\
-\mu & -\left(\hbar \omega_{q}^{0}+\mu\right)
\end{array}\right]\left(\begin{array}{l}
u \\
v
\end{array}\right)=\hbar \tilde{\omega}\left(\begin{array}{l}
u \\
v
\end{array}\right)
$$

with frequencies $\tilde{\omega}=\omega_{q}^{B}$ and $\tilde{\omega}=-\omega_{q}^{B}$, respectively. The negative frequency solution corresponds to excitations in the $-\vec{q}$ direction. With this substitution, Eqs. 2.10 and 2.11 are decoupled:

$$
\begin{aligned}
& \hbar \omega \alpha+i \hbar \frac{d \alpha}{d t}=\hbar \omega_{q}^{B} \alpha+\left(u_{q}-v_{q}\right) \frac{V}{2} \psi_{0} \\
& \hbar \omega \beta+i \hbar \frac{d \beta}{d t}=-\hbar \omega_{q}^{B} \beta+\left(u_{q}-v_{q}\right) \frac{V}{2} \psi_{0}
\end{aligned}
$$

These equations are identical to those of first-order perturbation theory for a single-particle Schrödinger equation, and thus the rates of growth of $|\alpha|^{2}$ and $|\beta|^{2}$ can be evaluated using Fermi's golden rule. $|\alpha|^{2}$ and $|\beta|^{2}$ are the probabilities for creating quasiparticles with momentum $\hbar \vec{q}$ and $-\hbar \vec{q}$, respectively.

The response of the condensate to Bragg scattering can be evaluated by calculating the momentum imparted to the condensate

$$
\langle\psi(\vec{r}, t)|\hat{p}| \psi(\vec{r}, t)\rangle=\hbar \vec{q} \times\left(|\alpha|^{2}-|\beta|^{2}\right)=\hbar \vec{q} \times\left(|u|^{2}-|v|^{2}\right)
$$

Normalizing by the Bragg scattering momentum $\hbar q$, we obtain the Bragg excitation rate per particle as

$$
\begin{aligned}
\frac{d}{d t}\left(\frac{\langle\psi(\vec{r}, t)|\hat{p}| \psi(\vec{r}, t)\rangle}{\hbar q N_{0}}\right) & =2 \pi \omega_{R}^{2}\left(u_{q}-v_{q}\right)^{2}\left[\delta\left(\hbar \omega-\hbar \omega_{q}^{B}\right)-\delta\left(\hbar \omega+\hbar \omega_{q}^{B}\right)\right] \\
= & 2 \pi \omega_{R}^{2}[S(\vec{q}, \omega)-S(-\vec{q},-\omega)]
\end{aligned}
$$

where, again, $\omega_{R}$ is the two-photon Rabi frequency.

This treatment reveals two important points. First, the mean-field treatment reproduces the suppression of the structure factor, even though, as discussed in Sec. 2.2.2, this suppression is indicative of correlations in the many-body condensate wavefunction. In the mean-field theory, correlations in the condensate wavefunction are explicitly neglected by the use of a Hartree wavefunction. Nevertheless, when the excitation of the condensate is also treated in a Hartree approximation (i.e. assuming all 
particles are in the same, albeit time-varying, single-particle wavefunction), the dynamic response is correctly obtained.

Second, this treatment takes into account both the positive and negative frequency terms in $V_{\bmod }$, unlike in Sec. 2.1.3 where we chose to consider only the positive frequency part for simplicity. One finds that the momentum imparted by stimulated scattering from the two Bragg beams measures the difference $S(\vec{q}, \omega)-S(-\vec{q},-\omega)$. This is important when one considers Bragg scattering from a non-zero temperature system which has thermally excited states and undergo anti-Stokes scattering. The dynamic structure factor for a non-zero temperature Bose-Einstein condensate is given as 20, 21]

$$
S(\vec{q}, \omega)=\left(u_{q}-v_{q}\right)^{2}\left[\left(\left\langle N_{q}^{B}\right\rangle+1\right) \delta\left(\hbar \omega-\hbar \omega_{q}^{B}\right)+\left\langle N_{-q}^{B}\right\rangle \delta\left(\hbar \omega+\hbar \omega_{q}^{B}\right)\right]
$$

where $\left\langle N_{q}^{B}\right\rangle$ is the thermal population of quasi-particles. Light scattering is thus quite sensitive to the presence of excitations in the condensate, as confirmed by the recent observation of superradiant light scattering from a condensate which is due to the buildup of excited particles in a preferred mode [32]. However, inserting Eq. 2.18 into Eq. 2.17 shows that the effects of thermally-excited particles are cancelled out in the Bragg scattering response, and thus one measures the zero-temperature structure factor even in a finite-temperature sample.

\subsubsection{The inhomogeneous condensate}

The Bose-Einstein condensates realized experimentally differ from the homogeneous condensates considered above due to their confinement. This confinement changes the Bragg scattering resonance from that predicted for a homogeneous Bose-Einstein condensate by introducing an inhomogeneous density distribution and by introducing Doppler broadening due to the zero-point momentum distribution.

The confining potential of magnetic or even optical traps is typically harmonic, taking the form $V(\vec{r})=(m / 2)\left(\omega_{x}^{2} x^{2}+\omega_{y}^{2} y^{2}+\omega_{z}^{2} z^{2}\right)$, where $\omega_{x}, \omega_{y}$, and $\omega_{z}$ are the trap frequencies. The condensate wavefunction $\psi(\vec{r})$ can be determined by the mean-field Gross-Pitaevskii equation [30, 31]. For large condensates for which the interaction energy is much larger than the kinetic energy, the wavefunction is given by the Thomas-Fermi solution as [3]

$$
|\psi(\vec{r})|^{2}=n \max \left(1-\left(\frac{x}{x_{c}}\right)^{2}-\left(\frac{y}{y_{c}}\right)^{2}-\left(\frac{z}{z_{c}}\right)^{2}, 0\right)
$$

where the Thomas-Fermi radii are defined as $x_{c}^{2}=2 \mu / m \omega_{x}^{2}$ (similar for $y_{c}$ and $z_{c}$ ), and $\mu=g n$ where $n$ is the maximum condensate density and $g=4 \pi \hbar^{2} a / m$ with $a$ being the $s$-wave scattering length.

Let us first consider the two effects of confinement separately:

a) Mean-field shift and broadening: The effects of interactions on Bragg scattering from an inhomogeneous density distribution can be accounted for using a local density approximation (discussed further in [25]). Using the density distribution of a condensate in the Thomas-Fermi regime (Eq. 2.19) and the predicted $S(\vec{q}, \omega)$ for a homogeneous condensate at the local value of the density, the Bragg resonance line shape is calculated to be [11]

$$
I_{\mu}(\omega) d \omega=\frac{15}{8} \frac{\omega^{2}-\omega_{q}^{0^{2}}}{\omega_{q}^{0}(\mu / \hbar)^{2}} \sqrt{1-\frac{\omega^{2}-\omega_{q}^{0}}{2 \omega_{q}^{0} \mu / \hbar}} d \omega
$$

The line strength $S(\vec{q})$ and center frequency can be obtained from Eq. 2.20 by integration. Explicitly, the static structure factor of a harmonically-confined Bose-Einstein condensate is given by

$$
S(\vec{q})=\frac{15 \eta}{64}\left(y+4 \eta-2 y \eta^{2}+12 \eta^{3}-3 y \eta^{4}\right)
$$

where $\eta^{2}=\hbar \omega_{q}^{0} / 2 \mu$ and $y=\pi-2 \arctan \left(\left(\eta^{2}-1\right) / 2 \eta\right)$. The line strength has the limiting values of $S(\vec{q}) \rightarrow 15 \pi / 32\left(\hbar \omega_{q}^{0} / 2 \mu\right)^{1 / 2}$ in the phonon regime and $S(\vec{q}) \rightarrow 1-4 \mu / 7 \hbar \omega_{q}^{0}$ in the 
free-particle regime. In accordance with the $f$-sum rule, the center frequency $\bar{\omega}$ is given as $\omega_{q}^{0} / S(\vec{q})$. In the free-particle regime $\left(\hbar \omega_{q}^{0} \gg \mu\right)$, the line center is shifted upwards from the free-particle resonance frequency by $4 \mu / 7 \hbar$, and broadened to an rms-width of

$$
\Delta \omega_{\mu}=\sqrt{\frac{8}{147}} \frac{\mu}{\hbar}
$$

b) Doppler broadening: Doppler broadening arises due to the initial momentum distribution of the condensate. The momentum distribution of a trapped Bose-Einstein condensate, assuming its full coherence, is given by the Fourier transform of the condensate wavefunction (Eq. 2.19). Thus, neglecting the mean-field shift (the impulse approximation as discussed in [25]), the Bragg excitation rate $I_{D}(\omega)$ from a Bose-Einstein condensate at a frequency difference of $\omega$ between the two Bragg beams is

$$
\begin{aligned}
& I_{D}(\omega) d \omega \\
& \quad \propto \int d^{3} \vec{k} \delta\left(\frac{\hbar \vec{k} \cdot \vec{q}}{m}-\left(\omega-\omega_{q}^{0}\right)\right)\left|\int d^{3} \vec{r} e^{-i \vec{k} \cdot \vec{r}} \psi(\vec{r})\right|^{2} d \omega \\
& \quad \propto \int d x_{1} d x_{2} e^{-i k(\omega) \cdot\left(x_{2}-x_{1}\right)} \int d y d z \psi^{*}\left(x_{1}, y, z\right) \psi\left(x_{2}, y, z\right) d \omega
\end{aligned}
$$

where $\hbar \vec{q}$ is the Bragg scattering momentum and $\psi(\vec{r})$ is the condensate wave function. In the last line, a coordinate system is chosen so that $\hbar \vec{q}$ lies in the $\hat{x}$-direction, and $k(\omega)=m\left(\omega-\omega_{q}^{0}\right) / \hbar q$ - In the Thomas-Fermi regime, for Bragg scattering along one of the principal axes of the harmonic trap, the Doppler line shape is then

$$
\begin{aligned}
& I_{D}(\omega) d \omega \propto \\
& \quad \frac{2\left(4+\kappa^{2}\right) J_{1}(\kappa) J_{2}(\kappa)+\kappa J_{0}(\kappa)\left[5 \kappa J_{1}(\kappa)-16 J_{2}(\kappa)+3 \kappa J_{3}(\kappa)\right]}{\kappa^{3}} d \omega
\end{aligned}
$$

where $\kappa=k(\omega) x_{c}$, and $J_{i}$ are Bessel functions. This line shape is similar to a Gaussian, but its rms-width is undefined. We therefore fitted a Gaussian function to the line shape and extracted an effective rms-width of

$$
\Delta \omega_{D} \simeq 1.58 \frac{\hbar q}{m x_{c}}
$$

\subsubsection{Relevance of Doppler broadening}

Thus, the Bragg scattering resonance for a trapped Bose-Einstein condensate is sensitive both to the velocity (Doppler shift) and the density (mean-field shift) of the atomic sample. The above treatments, in which we considered the effects of each of these shifts separately, are valid predictions in two limiting cases: for large condensates so that $\mu / \hbar \gg \hbar q / m x_{c}$ one can neglect Doppler broadening, while for small condensates where $\mu / \hbar \ll \hbar q / m x_{c}$ one can neglect mean-field broadening. However, in our experiments on Bragg scattering in the free-particle regime [10], the Doppler and mean-field widths were comparable, and one must consider both effects simultaneously.

We now show with the aid of simple sum rules that it is correct to add the Doppler broadening (Eq. 2.25) and the mean-field broadening (Eq. 2.22) in quadrature to obtain the total rms line width which can then be compared to experiments. A unified approach toward determining fully the dynamic structure factor in the presence of both Doppler and mean-field shifts has been presented recently by Zambelli et al. and was compared favorably with experimental data [25].

The condensate wavefunction $|g\rangle$ determined by the Gross-Pitaevskii equation is a solution of the equation $\mathcal{H}_{0}|g\rangle=\mu|g\rangle$ where

$$
\mathcal{H}_{0}=\frac{\hat{p}^{2}}{2 m}+U(\vec{r})+g n(\vec{r})
$$


In the regime $\hbar \omega_{q}^{0} \gg \mu$, the excitations relevant to Bragg scattering are well described as free-particle excitations which obey

$$
\hbar \omega_{f}|f\rangle=\left(\frac{\hat{p}^{2}}{2 m}+U(\vec{r})+2 g n(\vec{r})\right)|f\rangle=\mathcal{H}_{\text {exc }}|f\rangle
$$

This can be seen, for example, by considering Eq. 2.13 in the free-particle regime where $v_{q} \rightarrow 0$. Thus, the excitations are eigenfunctions of the Hamiltonian $\mathcal{H}_{\mathrm{exc}}=\mathcal{H}_{0}+g n(\vec{r})$ which is different from the Hamiltonian $\mathcal{H}_{0}$ which gives the condensate wavefunction [33]. The extra term $g n(\vec{r})$ represents the repulsion of excitations from the condensate which gives rise to the mean-field shift in the free-particle regime.

An exact determination of the resonance line shape using Fermi's golden rule requires detailed knowledge of the excitation wavefunctions. Such an explicit calculation has been performed 34] in the context of the two-photon optical excitation from the $1 S$ to the $2 S$ state in hydrogen which has been employed to probe properties of a hydrogen Bose-Einstein condensate [35, 36]. However, even without this exact description, moments of the spectral line can be easily determined. The first moment of the spectral line is given by

$$
\begin{aligned}
\hbar \bar{\omega} & =\frac{\int_{0}^{\infty} d \omega \omega S(\vec{q}, \omega)}{\int_{0}^{\infty} d \omega S(\vec{q}, \omega)} \\
& =\left\langle e^{i \vec{q} \cdot \vec{r}} \mathcal{H}_{\mathrm{exc}} e^{-i \vec{q} \cdot \vec{r}}-\mathcal{H}_{0}\right\rangle_{c} \\
& =\frac{\hbar^{2} q^{2}}{2 m}+g\langle n\rangle_{c}
\end{aligned}
$$

where $\langle X\rangle_{c}=\langle g|X| g\rangle$, and $\langle\vec{p}\rangle_{c}=0$ in the ground state. Thus the Bragg resonance line for a harmonically trapped condensate is shifted from the free-particle resonance by $g\langle n\rangle_{c}=4 \mu / 7 \hbar$.

The rms-width of the line $\Delta \omega=\sqrt{{\overline{\omega^{2}}}^{2}-\bar{\omega}^{2}}$ is calculated using

$$
\hbar^{2} \overline{\omega^{2}}=\left\langle e^{i \vec{q} \cdot \vec{r}} \mathcal{H}_{e x c}^{2} e^{-i \vec{q} \cdot \vec{r}}-\mathcal{H}_{0}^{2}\right\rangle_{c}
$$

by which one obtains

$$
\hbar^{2}(\Delta \omega)^{2}=\left\langle\left(\frac{\hbar \vec{q} \cdot \vec{p}}{m}\right)^{2}\right\rangle_{c}+g^{2}\left(\left\langle n^{2}\right\rangle_{c}-\langle n\rangle_{c}^{2}\right)
$$

Thus the total rms-width of the line is the sum of two widths in quadrature: the Doppler width due to the finite size of the condensate, and the line broadening due to the inhomogeneous condensate density.

\subsection{Experimental aspects of Bragg spectroscopy}

For the experimental studies of light scattering from Bose-Einstein condensates, condensates of atomic sodium were produced as in our previous experiments [1, 37]. These condensates typically contained $10^{7}$ atoms, and were held in a magnetic trap which provided a cylindrically-symmetric cigar-shaped harmonic confinement. The strength of the confining field could be easily modified by adjusting the currents through the magnetic trapping coils, allowing us to vary the peak condensate density in the range $(0.5-6) \times 10^{14} \mathrm{~cm}^{-3}$ and the radial half-width of the condensate $\left(x_{c}\right)$ between 7 and $15 \mu \mathrm{m}$.

The two laser beams used for Bragg scattering were derived from a common source, and had a detuning to the red of the $3 S_{1 / 2},|F=1\rangle \rightarrow 3 P_{3 / 2},\left|F^{\prime}=0,1,2\right\rangle$ optical transitions of about $\Delta=1.7$ $\mathrm{GHz}$ (similar parameters were used for experiments at NIST, Gaithersburg [14, 15, 38, 39]). A small frequency difference $\omega$ between the counter-propagating beams, on the order of $2 \pi \times 100 \mathrm{kHz}$, was introduced by using two independently-controlled acousto-optic modulators (AOM's) to control the frequency of two independent beams?

\footnotetext{
${ }^{2}$ In our study of the free-particle regime using counter-propagating Bragg beams, we alternately used a single AOM to generate a single beam with two different frequencies which was retro-reflected. This was done to minimize the deleterious effects of vibrations of optical components
} 


\begin{tabular}{c|c|c|c} 
& Phonon [1] & BEC & Free-particle [10] \\
\hline Velocity & $\hbar q / m=7 \mathrm{~mm} / \mathrm{s}$ & $c=10 \mathrm{~mm} / \mathrm{s}$ & $\hbar q / m=60 \mathrm{~mm} / \mathrm{s}$ \\
Energy & $\hbar \omega_{q}^{0}=h \times 1.5 \mathrm{kHz}$ & $\mu=h \times 6 \mathrm{kHz}$ & $\hbar \omega_{q}^{0}=h \times 100 \mathrm{kHz}$
\end{tabular}

Table 1. Conditions for Bragg scattering in the phonon and the free-particle regimes. The two regimes can be distinguished by comparing the Bragg scattering velocity $\hbar q / m$ to the speed of Bogoliubov sound $c=\sqrt{\mu / m}$, or, similarly, the Bragg scattering kinetic energy $\hbar \omega_{q}^{0}=\hbar^{2} q^{2} / 2 m$ to the chemical potential $\mu$. Bragg scattering using beams inclined by $14^{\circ}$ to one another accessed the phonon regime, while the use of counter-propagating beams accessed the free-particle regime.

To determine the Bragg scattering response, the laser beams were pulsed on for about $400 \mu \mathrm{s}$. After the optical excitation, the Bose-Einstein condensate was allowed to freely expand for $20-70 \mathrm{~ms}$ time of flight, after which the atoms were imaged by absorption imaging. Bragg scattered atoms were clearly distinguished by their displacement in the time-of-flight images (see Figs. 3, 8). The Bragg resonance spectrum for both trapped and untrapped Bose-Einstein condensates was determined by scanning the frequency difference $\omega$ and determining the fraction of Bragg scattered atoms.

As discussed above, there are two different regimes of excitations described by the Bogoliubov theory for the zero-temperature, weakly-interacting Bose-Einstein condensate: the phonon and the free-particle regimes. These two regimes can be distinguished in a number of equivalent ways. In the phonon regime, the wavevector of the excitation $q$ is smaller than the inverse healing length $q \ll \xi^{-1}=\sqrt{2 m \mu / \hbar^{2}}$. Equivalently, the free recoil velocity $\hbar q / m$ is smaller than the speed of sound $c$. One can also distinguish the free-particle from the phonon regime by comparing the free recoil energy $\hbar^{2} q^{2} / 2 m=\hbar \omega_{q}^{0}$ to the condensate interaction energy $\mu$ : $\hbar \omega_{q}^{0} \ll \mu$ in the phonon regime, and $\hbar \omega_{q}^{0} \gg \mu$ in the free-particle regime.

Either of these types of excitations can be accessed by Bragg scattering. In an $N$-th order Bragg scattering event induced by laser beams of wavevectors $\vec{k}_{1}$ and $\vec{k}_{2}\left(\left|\vec{k}_{1}\right| \simeq\left|\vec{k}_{2}\right|=k\right), N$ photons are transferred from one incident beam to another, imparting a momentum $\hbar q=2 N \hbar k \sin (\theta / 2)$, where $\theta$ is the angle between $\vec{k}_{1}$ and $\vec{k}_{2}$. Thus, small-angle Bragg scattering can be used to excite low-momentum phonon excitations, while large-angle or high-order Bragg scattering can excite highmomentum free-particle excitations.

The conditions in our two experiments are shown in Table f. In our first experimental study of Bragg scattering, excitations in the free-particle regime were studied by using two counter-propagating Bragg beams [10]. As shown in Table 1, the recoil velocity and energy for an excitation with a momentum of two photon recoils $(\hbar q=2 \hbar k)$ were clearly in the free-particle regime. In a second experiment, we accessed the phonon regime by using Bragg beams which were inclined at a small angle of about $14^{\circ}$ with respect to one another. This yielded a recoil velocity and energy which implied that Bragg scattering in a trapped Bose-Einstein condensate would occur in the phonon regime.

\subsection{Light scattering in the free-particle regime}

\subsubsection{Measurement of line shift and line broadening}

Fig. 3 illustrates our experimental method for probing the free-particle regime. Counter-propagating Bragg beams were incident along a radial direction (vertical in image) of the cigar-shaped condensate. After probing, the condensate was allowed to freely expand, allowing Bragg scattered atoms to separate spatially from the unscattered atoms, as shown in the figure. During free expansion, a cigar-shaped condensate expands primarily radially, with a maximum radial velocity of $v_{r}=\sqrt{2 \mu / m}=\sqrt{2} c$. Thus, since the Bragg scattered atoms are clearly separated from the remaining condensate during the time of flight, $\hbar q / m \gg c$ and thus Bragg scattering at a momentum of $\hbar q=2 \hbar k$ produces free-particle excitations.

The use of Bragg spectroscopy allowed the true momentum distribution of a gaseous Bose-Einstein condensate to be observed for the first time. Previous to this work, the onset of Bose-Einstein con- 


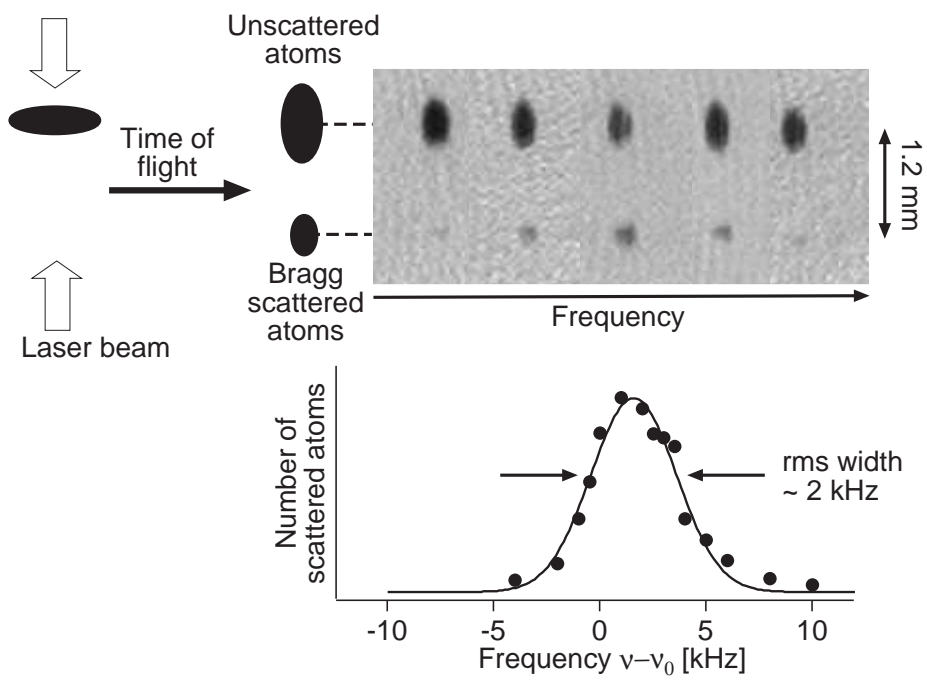

Fig. 3. Measuring the momentum distribution of a condensate [10]. Atoms were stimulated by two counterpropagating laser beams to absorb a photon from one beam and emit it into the other beam, resulting in momentum transfer to the atoms, as observed in ballistic expansion after $20 \mathrm{~ms}$ time of flight. The number of Bragg scattered atoms showed a narrow resonance when the difference frequency between the two laser beams was varied.

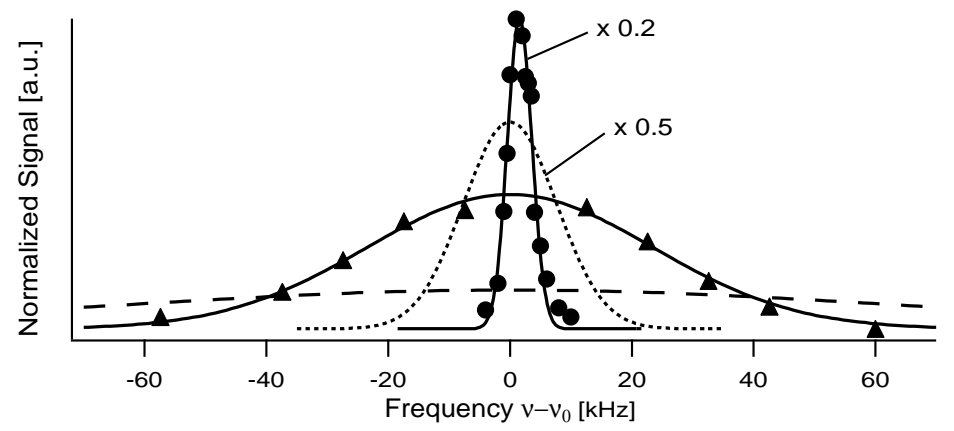

Fig. 4. Bragg resonances for a trapped condensate (circles) and after $3 \mathrm{~ms}$ time of flight (triangles). For comparison, the momentum distributions of the ground state of the trapping potential (dots) and of a $1 \mu \mathrm{K}$ cold, thermal cloud (dashes) are indicated.

\begin{tabular}{c|c|c|c|c} 
& thermal & ideal & interacting & time-of- \\
& cloud & BEC & BEC & flight \\
\hline$\Delta x$ & $a_{\text {osc }} \sqrt{k_{B} T / \hbar \omega}$ & $a_{\text {osc }}$ & $a_{\text {osc }} \xi$ & \\
$\Delta p$ & $\left(\hbar / a_{\text {osc }}\right) \sqrt{k_{B} T / \hbar \omega}$ & $\hbar / a_{\text {osc }}$ & $\left(\hbar / a_{\text {osc }}\right)(1 / \xi)$ & $\left(\hbar / a_{\text {osc }}\right) \xi$ \\
$\Delta x \Delta p / \hbar$ & $k_{B} T / \hbar \omega$ & 1 & 1 &
\end{tabular}

Table 2. Scaling of spatial and momentum widths of thermal clouds and Bose-Einstein condensates in an isotropic harmonic trap with frequency $\omega$. The parameter $\xi=\sqrt{\mu / \hbar \omega}$ denotes the strength of the mean-field interaction, and $a_{\mathrm{osc}}=\sqrt{\hbar / m \omega}$ the oscillator length. 

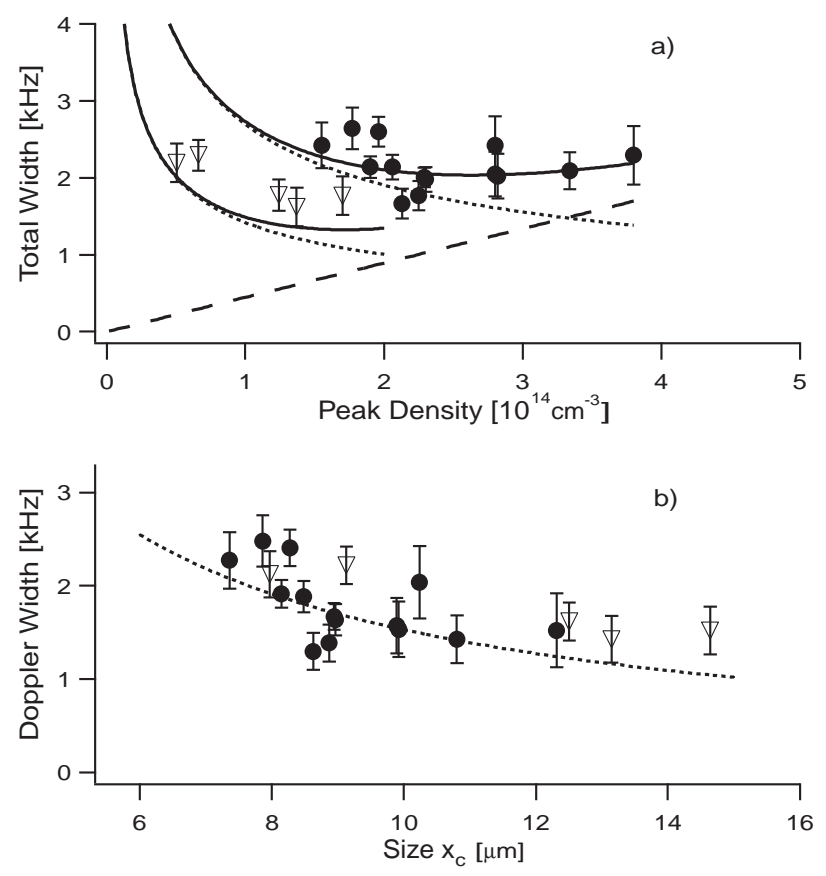

Fig. 5. Line width in Bragg spectroscopy. The widths are shown for various densities and sizes of the condensate using two different radial trapping frequencies, $\nu_{r}=(195 \pm 20) \mathrm{Hz}$ (circles), and $\nu_{r}=(95 \pm 20)$ $\mathrm{Hz}$ (triangles). The lines in a) show the contributions of the mean-field energy (dashed), due to the finite size (dotted, for both trapping frequencies), and their quadrature sum (solid lines). Fig. b) displays the Doppler width, obtained by subtracting the contributions of the mean-field and the finite pulse duration from the total width. The observed Doppler width of the resonance is proportional to the condensate's momentum uncertainty $\Delta p$, and was consistent with the Heisenberg limit $\Delta p \approx \hbar / x_{c}$ as the condensate radius $x_{c}$ was varied. Thus, the coherence length of the condensate is equal to its physical size, i.e. the condensate is one "coherent matter wave." An analogous measurement in the time domain has been done in Gaithersburg [38]. The error bars are $1 \sigma$ errors of the Gaussian fits to the data.

densation had been observed by the observation of a bimodal density distribution either in situ, or else in time-of-flight. In both cases, the condensate can be distinguished from the thermal cloud because its energy $\mu$, which is typically dominated by interaction energy, is smaller than the thermal energy $k_{B} T$. In situ, a separation between the two components of the gas occurs due to the inhomogeneous quadratic trapping potential, while in time-of-flight images, the separation is seen in velocity space after the explosive conversion of the interaction energy of the condensate to kinetic energy of ballistic expansion; in both cases, the spatial extent of the gas scales as the square root of its energy. As a result, both in time-of-flight and in the spatial domain, is the onset of BEC indicated by a narrowing of the density distribution relative to that of the thermal component by a factor of $\sqrt{\mu / k_{B} T}$ (Table 2). Indeed, we used Bragg spectroscopy to observe the expanding condensate in momentum space after its interaction energy had been converted to kinetic energy during its time of flight (Fig. (1). In a time-of-flight analysis, the condensate distribution is just $\approx 3$ times narrower than that of the thermal cloud (corresponding to the factor $\sqrt{\mu / k_{B} T}$ ), but wider than the momentum distribution of the harmonic oscillator ground state.

However, the in situ momentum distribution of the trapped condensate is much narrower than in ballistic expansion, by a factor $\xi^{2}=\mu / \hbar \omega$ (Table 2). It is also narrower than the momentum distribution of the harmonic oscillator ground state. This could be directly determined by the use of Bragg spectroscopy before releasing the condensate from its trap. As shown in Fig. 田, the momentum distribution of a trapped Bose-Einstein condensate is significantly narrower than that observed in 
time-of-flight; for the data shown, the in situ momentum width is 10 times narrower than in timeof-flight. Thus, the distinction between the thermal cloud and the condensate in momentum space is much more stark than in coordinate space and emphasizes the original description by Einstein of Bose-Einstein condensation as a condensation in momentum space.

We obtained Bragg resonance spectra for trapped condensates over a range of condensate densities and radial widths, and determined the shifts of the line center (Fig. 10) and the rms-widths (Fig. D) of the resonances. The shifts of the line center for trapped condensates from the free-particle resonance frequency were compared with measurements of the chemical potential determined from the width of condensates in time-of-flight images [1]. The measured shift of $(0.54 \pm 0.07) \mu / \hbar$ was in agreement with the predicted $0.57 \mu / \hbar$. In related work, mean-field line shifts measured by two-photon spectroscopy of atomic hydrogen were used as a measurement of the $a_{1 S, 2 S}$ scattering length for collisions between atoms in the $1 S$ and $2 S$ states [35].

The rms line widths showed effects of both mean-field broadening and Doppler broadening: meanfield broadening caused the line width to increase as the condensate number, and hence its size and density, were increased, while Doppler-broadening caused the rms-width to increase as the radial width of the condensate was decreased. Experimental Bragg spectra for conditions at the crossover between mean-field dominated (local density approximation) and Doppler dominated (impulse approximation) behaviour are shown in Fig. 5. These were analyzed in Ref. [25] and compared with a theoretical treatment based on an eikonal approximation which accounts for both effects.

The Doppler line widths were extracted from Bragg spectra by subtracting the broadening due to the mean-field and the finite pulse duration. Doppler widths were determined for both trapped condensates and condensates released from the trap. The width for freely-expanding condensates during the conversion of their interaction energy into kinetic energy was in full agreement with meanfield theory [40]. Thus, both in momentum space and in coordinate space [1], the predicted behaviour of freely-expanding Bose-Einstein condensates has been quantitatively verified, nicely confirming the mean-field description of large-amplitude dynamics of a condensate.

\subsubsection{A measurement of the coherence length of a Bose-Einstein condensate}

The Doppler width of the Bragg scattering resonance of trapped Bose-Einstein condensates measured their momentum distribution and thus measured their coherence length. By observing momentum distributions which were equal to the Heisenberg-limited momentum distribution determined by the condensate size (Fig. 5), our measurements showed that the coherence length of the condensate was no smaller than the radial width of the condensate, or simply that condensates can be described by a single macroscopic wavefunction.

To make this statement more quantitative, consider the possibility that the condensate is not fully coherent. As shown pictorially in Fig. 6, consider a situation in which the trapped Bose-Einstein condensate is actually composed of many smaller coherent condensates of typical size $\chi$ with no phase relation between them. Formally, one considers

$$
\left\langle\hat{\Psi}^{\dagger}\left(\vec{r}_{1}\right) \hat{\Psi}\left(\vec{r}_{2}\right)\right\rangle=\left\langle\hat{\Psi}^{\dagger}\left(\vec{r}_{1}\right)\right\rangle\left\langle\hat{\Psi}\left(\vec{r}_{2}\right)\right\rangle g^{(1)}\left(\left|\vec{r}_{1}-\vec{r}_{2}\right|\right)
$$

where $g^{(1)}(r)$ is the first-order coherence function which decays from $g^{(1)}(0)=1$ to $g^{(1)}(r \gg \chi)=0$. The Doppler line shape of the Bragg resonance, shown in Eq. 2.23 for the case of full coherence, now becomes

$$
\begin{aligned}
& I_{D}(\omega) d \omega \propto \\
& \quad \int d x_{1} d x_{2} e^{-i k(\omega)\left(x_{2}-x_{1}\right)} \int d y d z\left\langle\hat{\Psi}^{\dagger}\left(x_{1}, y, z\right) \hat{\Psi}\left(x_{2}, y, z\right)\right\rangle d \omega= \\
& \quad \int d x_{1} d x_{2} e^{-i k(\omega)\left(x_{2}-x_{1}\right)} g^{(1)}\left(\left|x_{1}-x_{2}\right|\right) \int d y d z \psi^{*}\left(x_{1}, y, z\right) \psi\left(x_{2}, y, z\right) d \omega
\end{aligned}
$$

The effect of a limited coherence length is to increase the momentum distribution and thereby broaden the Doppler width of the Bragg resonance. For example, consider a condensate wavefunction 


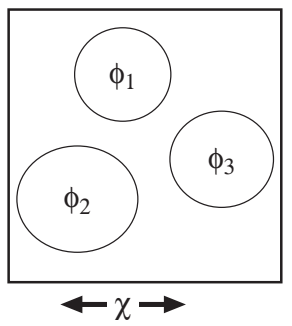

Fig. 6. A quasi-condensate can be depicted pictorially as being composed of many smaller condensates, each with a well-defined phase which is unrelated to the phase of the other small condensates. The range of phase coherence is the coherence length $\chi$.

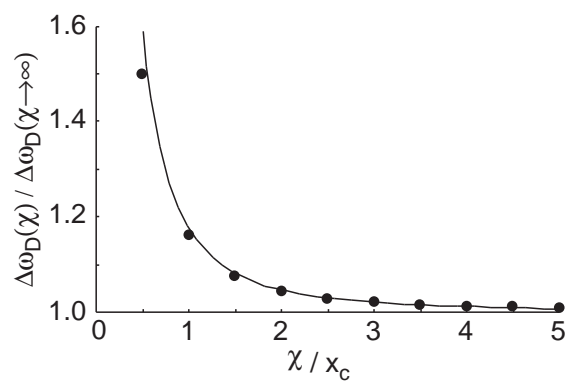

Fig. 7. Doppler width of the Bragg resonance as a function of the coherence length $\chi$. Shown is the ratio of the Doppler width of a partly coherent condensate to that of a fully coherent condensate, i.e. one for which $\chi \rightarrow \infty$. The coherence length is given in units of the Thomas-Fermi radius $x_{c}$. The results of a numerical calculation of Eq. 2.34 using a Thomas-Fermi wavefunction and $g^{(1)}(r)=\exp \left(-r^{2} / 2 \chi^{2}\right)$ (points) are well-described by the approximation of Eq. 2.35 (line).

which is Gaussian $\psi(\vec{r})=\exp \left(-\sum x_{i}^{2} / 2 \sigma_{i}^{2}\right)$ with rms-widths of $\sigma_{i},(i=x, y, z)$, and a first-order correlation function of the form $g^{(1)}(r)=e^{-r^{2} / 2 \chi^{2}}$. For Bragg scattering along the $\hat{x}$-direction, the Doppler line width becomes $\Delta \omega_{D}(\chi)=\hbar q / m \times \sqrt{\sigma_{x}^{-2}+\chi^{-2}}$. Fig. 7 shows the Doppler line width $\Delta \omega_{D}(\chi)$ calculated using the Thomas-Fermi condensate wavefunction for different values of the coherence length $\chi$. The calculations agree well with an approximation of the form

$$
\Delta \omega_{D}(\chi) \simeq \sqrt{\left[\Delta \omega_{D}(\chi \rightarrow \infty)\right]^{2}+\left[\frac{\hbar q}{m \chi}\right]^{2}}
$$

This relation can be used to extract a coherence length from the Doppler widths for trapped BoseEinstein condensates. Taking the average for all data points shown in Fig. 5b (assuming the ratio of the coherence length to the condensate size is constant), one finds an average value for the ratio of the measured Doppler width to that predicted for a fully coherent condensate of $\Delta \omega_{D}(\chi) / \Delta \omega_{D}(\chi \rightarrow \infty)=$ $1.09 \pm 0.11$. This corresponds to a determination of the coherence length in the range $\chi=1.4_{-0.5}^{+\infty} \times x_{c}$, i.e. the measurements place a lower bound on the coherence length of $\chi \simeq x_{c}$ and are consistent with full coherence $(\chi \rightarrow \infty)$.

The coherence of a condensate was first demonstrated by Andrews et al. by the observation of matter-wave interference when two independent Bose-Einstein condensates were overlapped 41]. The regular high-contrast fringes indicated, at least qualitatively, that the condensates possessed longrange first-order coherence. Our measurements using Bragg scattering provide a more quantitative measure of this property. Recently, two other groups have made measurements of the coherence length 38, 42]. These measurements give evidence of a decay of the coherence length over two length 


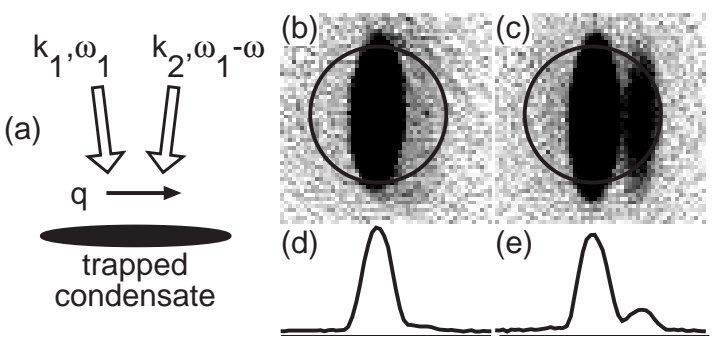

Fig. 8. Observation of momentum transfer by Bragg scattering. (a) Atoms were exposed to laser beams with wavevectors $\overrightarrow{k_{1}}$ and $\overrightarrow{k_{2}}$ and frequency difference $\omega$, imparting momentum $\hbar \vec{q}$ along the axis of the trapped condensate. The Bragg scattering response of trapped condensates (b,d) was much weaker than that of condensates after a $5 \mathrm{~ms}$ free expansion (c,e). Absorption images (b,c) after $70 \mathrm{~ms}$ time of flight show scattered atoms distinguished from the denser unscattered cloud by their axial displacement. Curves (d,e) show radially averaged (vertically in image) profiles of the optical density after subtraction of the thermal distribution. The Bragg scattering velocity is smaller than the speed of sound in the condensate (position indicated by circle). Images are $3.3 \times 3.3 \mathrm{~mm}$. Figure taken from Ref. [11].

scales. In particular, the Munich group ascribes the short-range decay of the first-order coherence function to the presence of thermal excitations. Instead of light, one could also use the scattering of fast atoms to determine the coherence length of a condensate, as suggested recently [43].

\subsection{Light scattering in the phonon regime}

\subsubsection{Experimental study}

A second experiment explored the use of Bragg spectroscopy of a Bose-Einstein condensate in the phonon regime [11. Laser beams separated by an angle of about $14^{\circ}$ were directed at a condensate so that the Bragg scattering momentum $\hbar \vec{q}$ was directed along the condensate axis (Fig. 8). Orienting the Bragg scattering momentum in the axial direction was necessary to allow for a clear distinction between the Bragg scattered atoms and the unscattered condensate in time-of-flight images, since the condensate expands radially in at a velocity $v_{r}=\sqrt{2} c$ which is greater than the recoil velocity $\hbar q / m$ in the phonon regime $(c>\hbar q / m)$. The axial expansion is slower than the radial expansion by a factor which is $2 / \pi$ times the aspect ratio of the cylindrical condensate [40], and was negligible for our experimental conditions.

We could directly compare Bragg scattering in the phonon and free-particle regimes by using the identical optical setup to optically excite either a trapped Bose-Einstein condensate, or a condensate that had been allowed to freely expand for $5 \mathrm{~ms}$ before the Bragg excitation. As discussed above, excitation in the trapped condensate occurred in the phonon regime. As for the expanded cloud, its density was reduced by a factor of 23 and thus the speed of sound by a factor of 5 from that of a trapped Bose-Einstein condensate. Therefore, excitations in expanded cloud occurred in the free-particle regime.

As shown in Figs. 8 and 9, the Bragg scattering response in the phonon regime was significantly weaker in strength and shifted upwards in frequency from that of free particles as predicted by Bogoliubov theory (Sec. 2.2). The line strength and center shift were measured for condensates at various densities, as presented in Fig. 10. As the condensate density is increased, the speed of sound increases and, at a constant Bragg scattering momentum $\hbar q$, one pushes further into the phonon regime where the line strength decreases. We could directly compare our experimental results to the predictions of the local density approximation (Eq. 2.20) since the Doppler shift was negligible at small momentum transfer. The striking difference from the free-particle regime demonstrates the collective nature of low momentum excitations.

The results summarized in Fig. 10 show that we have confirmed the Bogoliubov dispersion relation 
(Fig. 2) in both the small- and large-momentum regime. Data were taken at only two values of the momentum transfer, but by varying the condensate density, the ratio of the recoil energy to the mean-field energy could be smoothly varied.

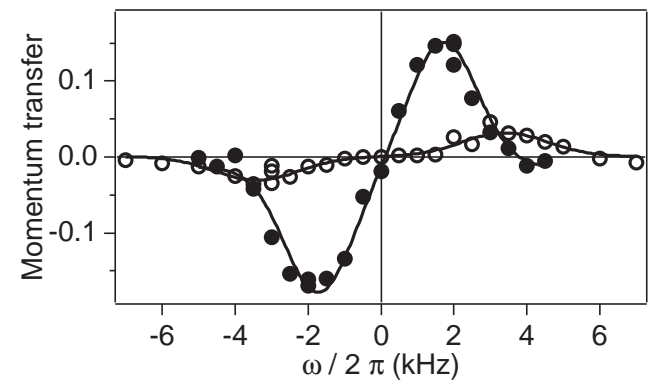

Fig. 9. Bragg scattering of phonons and of free particles. Momentum transfer per particle, in units of $\hbar q$, is shown vs. the frequency difference $\omega / 2 \pi$ between the two Bragg beams. Open symbols represent the phonon excitation spectrum for a trapped condensate at a chemical potential $\mu / h=9.2 \mathrm{kHz}$ (compared to the free recoil shift of $\approx 1.4 \mathrm{kHz}$ ). Closed symbols show the free-particle response of an expanded cloud. Lines are fits to the difference of two Gaussian line shapes representing excitation in the forward and backward directions.

\subsubsection{Suppression of light scattering from a Bose-Einstein condensate}

The diminished line strength for stimulated light scattering observed in the phonon regime shows that inelastic spontaneous light scattering (Rayleigh scattering) from a Bose-Einstein condensate is different from the light scattering from an equal number of non-interacting atoms. Consider Rayleigh scattering from a homogeneous Bose-Einstein condensate with a chemical potential $\mu$. Light scattered at an angle $\theta$ from an incident beam of light with wavevector $\vec{k}$ imparts a momentum of magnitude

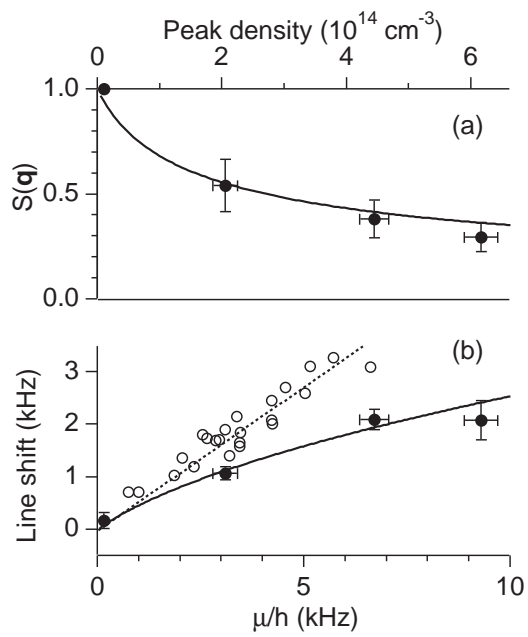

Fig. 10. Line strengths and line shifts of Bragg resonances. Shown are (a) the static structure factor $S(\vec{q})$ and (b) the shift of the line center from the free-particle resonance. $S(\vec{q})$ is the ratio of the line strength at a given chemical potential $\mu$ to that observed for free particles. As the density and $\mu$ increase, the structure factor is reduced, and the Bragg resonance is shifted upward in frequency. Solid lines are predictions of a local-density approximation for light scattering by $14^{\circ}$. The dotted line indicates a mean-field shift of $4 \mu / 7 \mathrm{~h}$ as measured in the free-particle regime using a scattering angle of 180 degrees. Figure taken from Ref. [11]. 


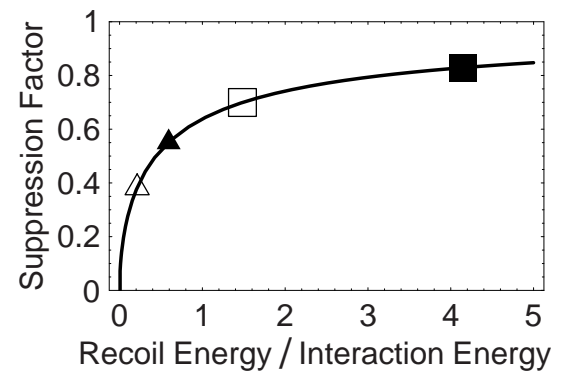

Fig. 11. Rayleigh scattering from a homogeneous interacting Bose-Einstein condensate is suppressed with respect to scattering from free atoms. The suppression factor is plotted against the ratio of the recoil energy $\hbar \omega_{k}^{0}=\hbar^{2} k^{2} / 2 m$ to the interaction energy $\mu$, where $k$ the wavevector of the incident light. Points indicate the factor by which the scattering of near-resonant light at $\lambda=589 \mathrm{~nm}$ (closed symbols) and infrared light at $\lambda=985 \mathrm{~nm}$ (open symbols) is suppressed at typical condensate densities attained in our magnetic $\left(n=4 \times 10^{14} \mathrm{~cm}^{-3}\right.$, squares) and optical $\left(n=3 \times 10^{15} \mathrm{~cm}^{-3}\right.$, triangles $)$ traps.

$\hbar q=2 \hbar k \sin (\theta / 2)$. The intensity of light scattered at this angle is diminished by $S(q)=\omega_{q}^{0} / \omega_{q}^{B}$. Integrating over all possible scattering angles $\theta$ and accounting for the dipolar emission pattern, we find that Rayleigh scattering from a homogeneous interacting Bose-Einstein condensate is suppressed by a factor

$$
\begin{aligned}
F= & \frac{3}{8 \pi} \int d \Omega\left(\cos ^{2} \theta+\sin ^{2} \theta \sin ^{2} \phi\right) \frac{2 k \sin (\theta / 2)}{\sqrt{(2 k \sin (\theta / 2))^{2}+2 \xi^{-2}}} \\
= & \frac{1}{64 x^{3} \sqrt{1+2 x}}\left[\sqrt{2 x}\left(15+46 x+64 x^{2}+64 x^{3}\right)\right. \\
& \left.-3 \sqrt{1+2 x}\left(5+12 x+16 x^{2}\right) \tanh ^{-1}\left(\sqrt{\frac{2 x}{1+2 x}}\right)\right]
\end{aligned}
$$

where $x=\hbar \omega_{k}^{0} / \mu$.

This suppression in Rayleigh scattering should be observable in current Bose-Einstein condensation experiments (Fig. 11). For example, Rayleigh scattering of near-resonant light from a sodium condensate at a density of $3 \times 10^{15} \mathrm{~cm}^{-3}$, which is the maximum density of condensates which has been obtained in optical traps [44], should be reduced by a factor of two. It would also be interesting to measure this suppression of light scattering at a Feshbach resonance 45, where the chemical potential can be made quite large by tuning the scattering length $a$ using magnetic fields. In such experiments, light scattering may allow one to study dynamically how the pair correlations in a condensate are established in response to a sudden increase in the interaction strength.

\section{Amplified scattering of light}

\subsection{Introduction}

In the previous section, we discussed the elementary process in which a photon is inelastically scattered from a condensate and creates a quasi-particle. We focused on the limit of low intensity of the probe light which means that each photon is assumed to probe the "original condensate." This is valid as long as the quasi-particles created by photon scattering decay or decohere fast enough so that the optical properties of the condensate don't change.

However, the low temperature and coherent nature of the condensate imply very long decoherence times, leading to long-lived quasi-particles which can affect the scattering of subsequent photons. The presence of long-lived excitations introduces strong correlations between successive Rayleigh scattering 
events 46]. This provides a positive feedback mechanism and leads to directional amplified Rayleigh scattering.

This non-linear behavior happens already at very low laser intensity. The amplification of the scattering process is stimulated by the matter-wave field analogous to stimulated emission in the optical laser. When this amplification process starts spontaneously, a new form of superradiance is realized (Sect. 3.2). When it is initiated by seeding the condensate with an input matter wave, it leads to coherent atom amplification (Sect. 3.3).

\subsection{Superradiant Rayleigh scattering}

\subsubsection{Semiclassical derivation of the gain mechanism}

The gain mechanism for Rayleigh scattering from a condensate can be derived semi-classically. When a condensate of $N_{0}$ atoms is exposed to a laser beam with wavevector $\vec{k}_{0}$ and scatters a photon with wavevector $\vec{k}_{i}$, an atom (or quasi-particle, also called momentum side-mode in [47) with recoil momentum $\hbar \vec{K}_{j}=\hbar\left(\vec{k}_{0}-\vec{k}_{i}\right)$ is generated. Since light propagates at a velocity about ten orders of magnitude greater than the atomic recoil velocity $(3 \mathrm{~cm} / \mathrm{s}$ for sodium), the recoiling atoms remain within the volume of the condensate long after the photons have left and affect subsequent scattering events. They interfere with the condensate at rest to form a moving matter wave grating of wavevector $\vec{K}_{j}$, which diffracts the laser beam into the phase-matching direction $\vec{k}_{i}\left(=\vec{k}_{0}-\vec{K}_{j}\right)$. This diffraction is a self-amplifying process because every diffracted photon creates another recoiling atom which further increases the amplitude of the matter wave grating.

When $N_{j}$ recoiling atoms interfere with $N_{0}$ condensate atoms, the density modulation comprises $N_{\text {mod }}=2 \sqrt{N_{0} N_{j}}$ atoms. The light scattered by these atoms interferes constructively in the phasematching direction with a total power $P$ of

$$
\begin{aligned}
P & =\hbar \omega f_{j} R \frac{N_{\mathrm{mod}}^{2}}{4} \\
f_{j} & =\frac{\sin ^{2} \theta_{j}}{8 \pi / 3} \Omega_{j} .
\end{aligned}
$$

Here, $R$ is the rate for single-atom Rayleigh scattering which is proportional to the laser intensity, and $\omega$ is the frequency of the radiation. The angular term in Eq. 3.2 reflects the dipolar emission pattern with $\theta_{j}$ being the angle between the polarization of the incident light and the direction of emission. Due to the finite size of the sample, the phase matching condition is fulfilled over the solid angle $\Omega_{j} \sim \lambda^{2} / A$, where $A$ is the cross-sectional area of the condensate perpendicular to the direction of the light emission and $\lambda$ the optical wavelength. More rigorously, $\Omega_{j}$ is given by the usual phase-matching integral for superradiance in extended samples 48]:

$$
\Omega_{j}=\int d \Omega(\vec{k})\left|\int \tilde{\rho}(\vec{r}) \exp ^{i\left(\vec{k}_{i}-\vec{k}\right) \cdot \vec{r}} d \vec{r}\right|^{2},
$$

where $|\vec{k}|=\left|\vec{k}_{i}\right|$, and $\tilde{\rho}(\vec{r})$ is the normalized density distribution of the condensate $\left(\int \tilde{\rho}(\vec{r}) d \vec{r}=1\right)$. Since each scattered photon creates a recoiling atom, we obtain the growth rate for $N_{j}$ from $P / \hbar \omega$ :

$$
\dot{N}_{j}=G_{j} N_{j}
$$

This equation predicts exponential growth of $N_{j}$ with the small-signal gain $G_{j}=R N_{0} f_{j} \sim$ $R \sin ^{2} \theta_{j} D_{j}$, where $D_{j} \sim \rho_{0} \lambda^{2} l_{j}$ is the resonant optical density for a condensate with an atomic density $\rho_{0}$ and a length $l_{j}$ along the axis of emission. Therefore, for an anisotropic Bose condensate, the gain is largest when the light is emitted along its longest axis (the "end-fire mode" 49.). Eq. (3.4) is valid in the absence of decoherence and predicts the build-up of highly anisotropic Rayleigh scattering from a non-spherical sample of atoms. 
The built-up of a matter-wave grating dramatically changes the optical properties of the condensate - the condensate becomes "reflective." This property is only due to the coherent nature of the condensate. This phenomenon is completely different from the reflectivity caused by polaritons which depends on the dipole-dipole interaction of excited atoms and requires high atomic densities [8, 9].

\subsubsection{Four-wave mixing of light and atoms}

Further insight is obtained by describing the scattering process fully quantum-mechanically. As discussed in Sect. 2.1.1, the Hamiltonian $\mathcal{H}^{\prime}$ describes light scattering as a coupling (i.e. a four-wave mixing) between two Schrödinger (matter) waves and two electromagnetic waves. It is convenient to rewrite $\mathcal{H}^{\prime}$ by choosing to quantize the recoiling atoms in the volume of the condensate and use plane waves for the light. The important terms in $\mathcal{H}^{\prime}$ include the macroscopically occupied initial atom field $\hat{a}_{0}$ (the condensate) and electromagnetic field $\hat{c}_{0}$ (the incident laser beam with wavevector $\vec{k}_{0}$ ),

i.e. $\mathcal{H}^{\prime} \simeq C_{i, j} \hat{c}_{i}^{\dagger} \hat{a}_{j}^{\dagger} \hat{c}_{0} \hat{a}_{0}$ where $\vec{k}_{i}$ is the wavevector of the outgoing light, and $\hat{a}_{j}^{\dagger}$ is the creation operator for an atom in the final state $j$.

The square of the matrix element which describes the creation of recoiling atoms is proportional to $N_{j}+1$ reflecting bosonic stimulation in the atomic field, where $N_{j}$ is the number of atoms in the final state $j$. We use Fermi's Golden Rule to sum over all final states of the electromagnetic field which are assumed to be initially empty (spontaneous scattering) and obtain the growth rate of $N_{j}$ as

$$
\begin{aligned}
\dot{N}_{j} & =G_{j}\left(N_{j}+1\right) \\
& =R N_{0} \frac{\sin ^{2} \theta_{j}}{8 \pi / 3} \Omega_{j}\left(N_{j}+1\right),
\end{aligned}
$$

which is identical to the classical treatment of Sect. 3.2.1 except that $N_{j}$ is replaced by $N_{j}+1$. The solid angle factor $\Omega_{j}$ now reflects the number of electromagnetic plane wave modes $i$ which are excited together with a quasiparticle in state $j$ localized in the volume of the condensate. Energy conservation requires that the frequency of the scattered light be red-shifted with respect to the incident radiation by the kinetic energy of the recoiling atoms. Eq. (3.5) describes both normal Rayleigh scattering at a constant total rate $\Sigma \dot{N}_{j}=R N_{0}$ when $N_{j} \ll 1$, and exponential gain of the $j$-th recoil mode due to bosonic stimulation once $N_{j}$ becomes non-negligible. Initially, the angular distribution of the scattered light follows the single-atom spontaneous (dipolar) emission pattern, but can become highly anisotropic when stimulation by the atomic field becomes important.

The choice of plane waves for the scattered light is arbitrary. Another basis set are spherical waves [49]. For the situation considered here it is more convenient to choose orthogonal modes for the different directions of light emission which have diffraction limited beam waists $A_{j}$ identical to the cross section of the condensate perpendicular to the axis of light emission (see Ref. [49], page 45). Therefore, for scattering into a specific direction, one has to consider only the longitudinal "beam waist modes" along the direction of scattering, and their wavevector is determined by energy conservation. Of course, the final result is the same (Eq. 3.5). The solid angle factor $\Omega_{j} \propto 1 / A_{j}$ appears in this derivation because the energy density of a single photon in the beam waist is inversely proportional to $A_{j}$.

One can account for decoherence and losses by adding a damping term to Eq. 3.5.

$$
\dot{N}_{j}=G_{j}\left(N_{j}+1\right)-D_{j} N_{j} .
$$

As we discuss below, this damping term reflects the finite linewidth of the Bragg resonance and is necessary to account for the observed threshold behavior.

\subsubsection{Bosonic stimulation by scattered atoms or scattered light?}

In the previous discussion, we evaluated coupling matrix elements assuming that the atoms scatter light always into empty modes of the electromagnetic field, and thus found that bosonic stimulation by the occupied atomic recoil mode was responsible for superradiant light scattering. If the time between 
light scattering events from the condensate is much longer than the time it takes the scattered photons to leave the condensate, this assumption is clearly justified. But suppose that the Rayleigh scattering rate were increased so that the mean number of scattered photons in the condensate were non-zero. What effect would this have on the observed superradiance? Does the number of photons occupying the scattered light mode lead to additional bosonic stimulation?

To answer this question and, in particular, to clarify the importance of the decay times of the atom and optical modes, let us consider light scattering into just one selected electromagnetic mode which is defined as the mode of a cavity built around the condensate. In the so-called bad cavity limit, we will obtain the results of the previous section.

The two modes of the light and the atoms are labeled as before and coupled by the operator

$$
\mathcal{H}^{\prime}=C \hat{c}_{i}^{\dagger} \hat{a}_{j}^{\dagger} \hat{c}_{0} \hat{a}_{0}+\text { h.c. }
$$

where $C$ denotes the strength of the coupling. In the interaction picture, we obtain coupled equations of motion for the mode operators

$$
\begin{aligned}
\frac{\mathrm{d} \hat{a}_{j}}{\mathrm{~d} t} & =-\frac{D_{a}}{2} \hat{a}_{j}-\left(\mathrm{i} C \hat{a}_{0} \hat{c}_{0}\right) \hat{c}_{i}^{\dagger} \\
\frac{\mathrm{d} \hat{c}_{i}^{\dagger}}{\mathrm{d} t} & =\left(\mathrm{i} C \hat{a}_{0}^{\dagger} \hat{c}_{0}^{\dagger}\right) \hat{a}_{j}-\frac{D_{c}}{2} \hat{c}_{i}^{\dagger}
\end{aligned}
$$

$D_{a}$ and $D_{c}$ are the damping rates for the number of atoms in mode $j$ and for the number of photons in mode $i$. Similar coupled equations were considered in Refs. [47,50]. If the scattered light is strongly damped we can adiabatically eliminate it by setting the l.h.s. of Eq. 3.9 to zero

$$
\hat{c}_{i}^{\dagger}=\frac{2 \mathrm{i} C}{D_{c}} \hat{a}_{0}^{\dagger} \hat{c}_{0}^{\dagger} \hat{a}_{j}
$$

Inserting this expression into Eq. 3.8 leads to a gain equation for the atomic field operator

$$
\frac{\mathrm{d} \hat{a}_{j}}{\mathrm{~d} t}=\frac{1}{2}\left[\frac{4|C|^{2}}{D_{c}} N_{0} n_{0}-D_{a}\right] \hat{a}_{j}
$$

The field operators for the initial states were replaced by the square root of the occupation numbers $N_{0}$ and $n_{0}$ for the atoms in the condensate and the photons in the laser beam which leads to an expression for the gain $G=4\left(|C|^{2} / D_{c}\right) N_{0} n_{0}$.

Alternatively, we can find the eigenvalues $\lambda_{1,2}$ of the matrix in Eqs. 3.8 and 3.9 as

$$
\lambda_{1,2}=-\frac{D_{a}+D_{c}}{4} \pm \sqrt{\left(\frac{D_{a}-D_{c}}{4}\right)^{2}+C^{2} N_{0} n_{0}}
$$

For $C=0$, the two eigenvalues are $-D_{a} / 2$ and $-D_{c} / 2$. When $D_{c} \gg D_{a}, G$, the two eigenvalues are $-D_{c} / 2$ and $\frac{1}{2}\left[\left(4|C|^{2} / D_{c}\right) N_{0} n_{0}-D_{a}\right]$; this is the result obtained in Eq. 3.11 .

Eqs. 3.8 and 3.9 can be applied to the free-space situation discussed in the previous section where the optical modes $i$ are the beam-waist modes quantized in a longitudinal box of length $L$ and mode density $L / c$. The rate of spontaneous emission into the beam waist mode is given by Fermi's golden rule and is proportional to the square of the matrix element times the mode density $|C|^{2} N_{0} n_{0}(L / c)$ and is equivalent to Eq. 3.5.

On resonance, a cavity of finesse $F$ enhances the density of states by the factor $8 F / \pi$ [51]. The cavity walls create mirror images of the radiating atoms which enhance the scattering rate. The number of effective mirror images is proportional to the finesse of the cavity. Therefore, the gain in Eq. 3.11 is proportional to the rate of spontaneous emission into the solid angle $\Omega_{j}$ and to the finesse of the cavity.

$$
G=R N_{0} \frac{\sin ^{2} \theta_{j}}{8 \pi / 3} \Omega_{j} \frac{8 F}{\pi}
$$


arXiv:cond-mat/0005001

The free-space limit is retrieved by setting the finesse to about unity $(F=\pi / 8)$ which cancels the last factor in Eq. 3.13. Or equivalently, free space corresponds to the limit of a cavity with a damping time of about $L / c$.

This result is useful to discuss bosonic stimulation by the matter wave field and the optical field. The operator describing light scattering (Eq. 3.7) has a matrix element squared of $|C|^{2}\left(N_{j}+1\right)\left(n_{i}+\right.$ 1) $N_{0} n_{0}$. Since this expectation value is proportional to $\left(N_{j}+1\right)\left(n_{i}+1\right)$, it seems that there is bosonic stimulation by both the final atomic state (occupied by $N_{j}$ atoms) and the final optical state (occupied by $n_{i}$ photons). However, Eqs. 3.11 and 3.13 show that this notion is incorrect. After adiabatic elimination of the rapidly-damped scattered light we have only stimulation by the atomic field. The superradiant gain $G$ is increased by increasing the finesse of the optical cavity, thus increasing the decay time of the scattered light. However, the gain does not explicitly depend on the number of photons in this mode.

Since the coupled equations have complete symmetry between light and atoms, one can also discuss the complementary case where the atoms are rapidly damped and are adiabatically eliminated. In this case, one obtains a gain equation for the scattered light showing optical stimulation, but not stimulation by the matter wave field in the final state. This situation applies to the optical laser and also to stimulated Brioullin scattering (see also Sect. 3.2.5).

On first sight it appears counterintuitive that bosonic stimulation is not multiplicative if we have a large occupation in both the final photon state and the final atomic state. However, when we have occupancy in all final states, the scattering can go both ways. Thus, let us consider the situation in which there are $N_{j}$ recoiling atoms and $n_{i}$ scattered photons in the volume of the condensate, and look at time scales much longer than the coherence time, i.e. a situation in which rate equations apply. The process $\left|n_{0}, n_{i} ; N_{0}, N_{j}\right\rangle \rightarrow\left|n_{0}-1, n_{i}+1 ; N_{0}-1, N_{j}+1\right\rangle$ by which atoms scatter out of the condensate into the recoil mode $j$ occurs with a rate proportional to the square of the matrix element $|C|^{2}\left(N_{j}+1\right)\left(n_{i}+1\right) N_{0} n_{0}$. The process $\left|n_{0}, n_{i} ; N_{0}, N_{j}\right\rangle \rightarrow\left|n_{0}+1, n_{i}-1 ; N_{0}+1, N_{j}-1\right\rangle$ by which atoms scatter back into the condensate has a rate proportional to $|C|^{2} N_{j} n_{i}\left(N_{0}+1\right)\left(n_{0}+1\right)$. The net rate of scattering atoms from the condensate into mode $j$ is the difference of the two partial rates. If we assume $N_{0}, N_{j}, n_{0} \gg 1$ and also much larger than $n_{i}$, since the scattered light was assumed to suffer the strongest damping, we obtain the leading term in the net scattering rate to be proportional to $|C|^{2} N_{j} N_{0} n_{0}$ as before. The bosonic stimulation by the least populated field (mode $i$ ) in the matrix element dropped out when the net rate was calculated.

This finding should be generally applicable. For example, when two condensed atoms form molecules by photoassociation or near a Feshbach resonance, the coupled equations involve a matter wave field for the atoms and for the molecules [52, 53] which leads to bosonic stimulation in the coupling matrix element and therefore in the partial rate. However, molecules are the most rapidly damped mode. Therefore the net rate of molecule formation is independent of bosonic stimulation by the particles in the final state and can not distinguish between a coherent molecular matter wave field and an incoherent classical ensemble of molecules in the final state.

Eq. 3.8 also describes Bragg scattering where a condensate is exposed to two strong laser beams in modes 0 and $i$. This results in Rabi oscillations of the atoms between states 0 and $j$. For short times $t$, one has $N_{j} \propto N_{0} n_{0} n_{i} t^{2}$. The scattering rate $\dot{N}_{j}$ is now proportional to $N_{j}^{1 / 2}$ which is incompatible with the simple concept of bosonic stimulation. The simple picture of bosonic stimulation is only obtained when we are in the regime where rate equations apply. This example shows that one has to be careful not to over-interpret the intuitive concept of bosonic stimulation. It can be misleading to use this concept in the case of coherently coupled fields. It can be applied to the situation where one of the fields (either the atomic or the optical one) is strongly damped, but then there is bosonic stimulation of the net scattering rate only by the long-lived field.

\subsubsection{Observation of directional emission of light and atoms}

For the experimental study of directional Rayleigh scattering, elongated Bose-Einstein condensates were prepared in a magnetic trap [37]. The trapped condensates were approximately $20 \mu \mathrm{m}$ in diameter $d$ and $200 \mu \mathrm{m}$ in length $l$ and contained several million sodium atoms in the $F=1$ hyperfine ground 


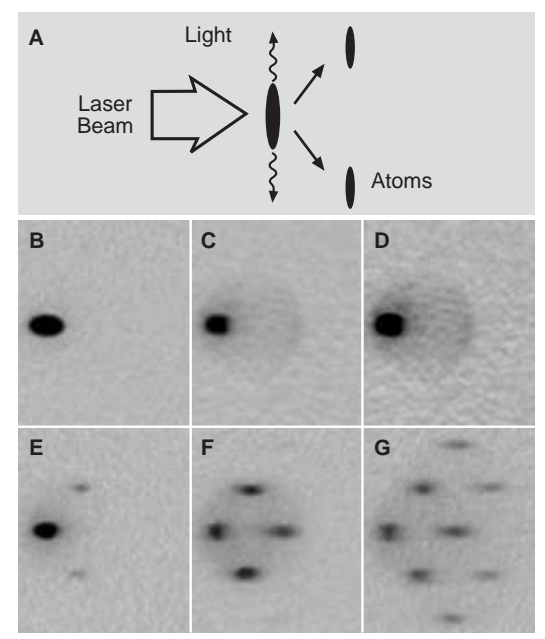

Fig. 12. Observation of superradiant Rayleigh scattering. (A) An elongated condensate is illuminated with a single off-resonant laser beam. Collective scattering leads to photons scattered predominantly along the axial direction, and atoms at 45 degrees. (B-G) Absorption images after $20 \mathrm{~ms}$ time-of-flight show the atomic momentum distribution after their exposure to a laser pulse of variable duration. When the polarization was parallel to the long axis, superradiance was suppressed, and normal Rayleigh scattering was observed (B-D). For perpendicular polarization, directional superradiant scattering of atoms was observed (E-G), and evolved to repeated scattering for longer laser pulses $(\mathrm{F}, \mathrm{G})$. The pulse durations were $25(\mathrm{~B}), 100$ (C,D), 35 (E), 75 $(\mathrm{F}), 100 \mu \mathrm{s}(\mathrm{G})$. The field of view of each image is $2.8 \times 3.3 \mathrm{~mm}$. The scattering angle appears larger than 45 degrees due to the angle of observation. All images use the same gray scale except for (D), which enhances the small signal of Rayleigh scattered atoms in (C). Reprinted with permission from Ref. [32], copyright 1999 American Association for the Advancement of Science.

state. The condensate was exposed to a single off-resonant laser pulse which was red-detuned by $1.7 \mathrm{GHz}$ from the $3 S_{1 / 2}, F=1 \rightarrow 3 P_{3 / 2}, F=0,1,2$ transition. The beam had a diameter of a few millimeters, propagated at an angle of 45 degrees to the vertical axis and intersected the elongated condensate perpendicular to its long axis (Fig. 12). Typical laser intensities were between 1 and $100 \mathrm{~mW} / \mathrm{cm}^{2}$ corresponding to Rayleigh scattering rates of 45 to $4500 \mathrm{~s}^{-1}$, and the pulse duration between 10 and $800 \mu \mathrm{s}$. In order to probe the momentum distribution of scattered atoms, the magnetic trap was suddenly turned off immediately after the light pulse, and the ballistically expanding cloud was imaged after 20 to $50 \mathrm{~ms}$ time-of-flight using resonant probe light propagating vertically onto a CCD camera.

The momentum distributions of atoms after light scattering (Fig. 12 B-G) showed a dramatic dependence on the polarization of the incident laser beam. For polarization parallel to the long axis of the elongated condensate $\left(\theta_{j}=0\right)$, light emission into the end-fire mode was suppressed, and the distribution of atoms followed the dipolar pattern of normal Rayleigh scattering. For perpendicular polarization $\left(\theta_{j}=\pi / 2\right)$, photons were predominantly emitted along the long axis of the condensate where the superradiant gain was the largest. The recoiling atoms appeared as highly directional beams propagating at an angle of 45 degrees with respect to this axis.

A characteristic feature of superradiance is an accelerated decay of the initial state. In our experiment, normal exponential decay and the superradiant decay could be directly compared by tracing the number of atoms remaining in the condensate at rest after exposure to light of different polarizations. For parallel polarization, we observed a simple exponential decay with the expected Rayleigh scattering rate (Fig. 13). For perpendicular polarization, the end-fire mode was active and the condensate decayed non-exponentially with a strongly accelerated superradiant rate.

The directional scattering of atoms implies that the light is scattered preferentially into the axial direction. This was verified by observing the scattered light with a CCD camera (Fig. 14 A). The 


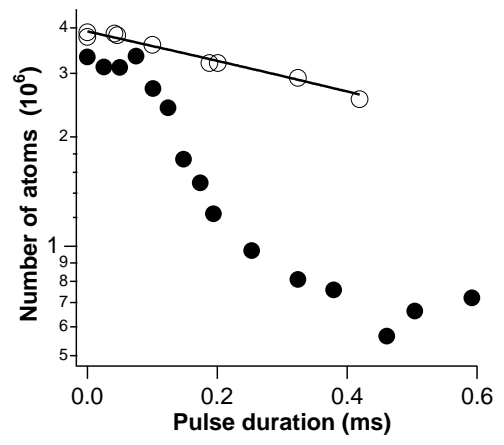

Fig. 13. Exponential and superradiant decay. The decay of atoms in the condensate at rest showed the normal exponential decay for parallel polarization (open circles) and faster superradiant decay for perpendicular polarization (full circles). The laser intensities $\left(13 \mathrm{~mW} / \mathrm{cm}^{2}\right)$ and oscillator strengths were equal in both cases. Reprinted with permission from Ref. [32], copyright 1999 American Association for the Advancement of Science.

camera was positioned out of focus of the imaging system, so that the images represent the angular distribution of photons emitted around the axial direction. The images consisted of bright spots with angular widths equal to the diffraction limit for a source with a diameter $\sim 14 \mu \mathrm{m}$. Typical images showed more than one such spot, and their pattern changed randomly under the same experimental conditions. The observation of a few spots is consistent with a Fresnel number $F=\pi d^{2} / 4 l \lambda$ slightly larger than one, implying that the geometric angle $d / l$ is larger than the diffraction angle $\lambda / d . F>1$ leads to multimode superradiance [54] since there is now more than one end-fire mode.

By replacing the camera with a photomultiplier, a time-resolved measurement of the scattered light intensity was obtained (Fig. 14 B). Simple Rayleigh scattering would give a constant signal during the square-shaped laser pulse. Instead, we observed a fast rise and a subsequent decay consistent with a stimulated process. Measurements at variable laser intensities showed a threshold for the onset of superradiance, and a shorter rise time for higher laser intensities. This behavior can be accounted for by Eq. 3.6.

We determined the exponential rate $\left(G_{j}-D_{j}\right)$ by fitting the initial rise in the light intensity. At early times the depletion of the condensate is negligible and $G_{j}$ and $D_{j}$ are constants. Fig. $14 \mathrm{C}$ shows the inverse rise time $\dot{N} / N$ vs. the Rayleigh scattering rate $R$, which was measured by "switching off" the superradiance using parallel polarization. The slope gives $G_{j} / R$ and the offset determines the loss $L_{j}$. The agreement between the calculated value for $G_{j} / R \sim 890$ (using $\Omega_{j} \sim 1.9 \times 10^{-4}$ and $N_{0}=4.7 \times 10^{6}$ ) and the result of the simple linear fit (790) is better than the uncertainty in the Rayleigh scattering rate (40\%). The offset in Fig. 14 C determines the threshold for superradiance and yields $1 / D_{j}=35 \mu s$.

The rate of decoherence $D_{j}$ for the superradiance indicates the decay of the matter wave interference. This has been studied separately using stimulated Rayleigh scattering (or Bragg spectroscopy) [10], where the linewidth of the Bragg resonance resulted from Doppler and mean-field broadening. The observed FWHM of approximately $5 \mathrm{kHz}$ yields a decoherence time of $32 \mu \mathrm{s}$, in good agreement with the value shown above.

For higher laser powers, a distinct change in both the momentum pattern of the atoms (Fig. 12 F,G) and in the photomultiplier traces (Fig. 14B) was observed. The atomic pattern showed additional momentum peaks which can be explained as a sequential scattering process where atoms in the initial momentum peak undergo further superradiant scattering. These processes are time-delayed with respect to the primary process, and showed up as a second peak in the time-resolved photomultiplier traces (Fig. 14B). Those higher-order peaks may also be affected by stimulated Raman scattering and four-wave mixing between matter waves [39] which couple the different recoil modes. 


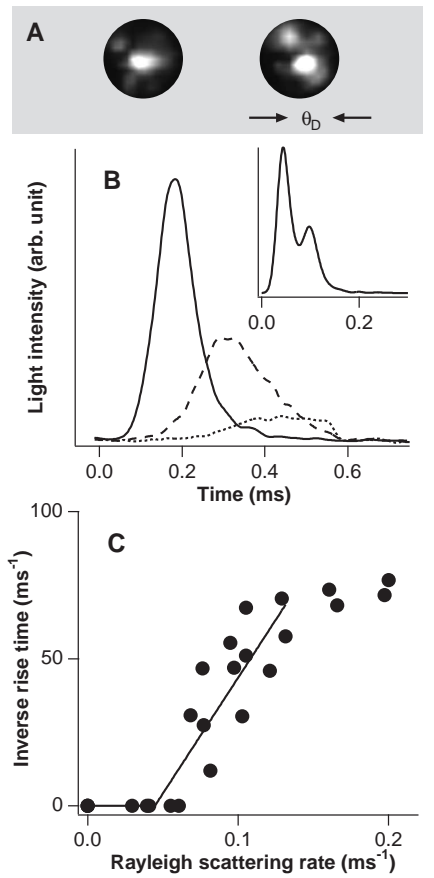

Fig. 14. Observation of directional emission of light. (A) The angular pattern of the emitted light along the axial direction showed a few bright spots with an angular width $\theta_{D}\left(1 / e^{2}\right.$ diameter) of $107 \pm 20 \mathrm{mrad}$, corresponding to the diffraction limited angle of an object of $\sim 14 \mu \mathrm{m}$ in diameter. The images were integrated over the entire duration of the light pulse. (B) The temporal evolution of the light intensity showed a strong initial increase characteristic of a stimulated process. For higher laser power, the pulse was shorter and more intense. The laser intensities were 3.8 (solid line), 2.4 (dashed line), and $1.4 \mathrm{~mW} / \mathrm{cm}^{2}$ (dotted line), and the duration was $550 \mu \mathrm{s}$. The inset shows a double-peak in the temporal signal when the laser intensity was about $15 \mathrm{~mW} / \mathrm{cm}^{2}$, which was above the threshold for sequential superradiant scattering. The photomultiplier recorded the light over an angle of $200 \mathrm{mrad}$ around the axial direction. (C) The dependence of the inverse initial rise time on the Rayleigh scattering rate shows a threshold for the stimulated process. The solid curve is a straight-line fit. Reprinted with permission from Ref. 32], copyright 1999 American Association for the Advancement of Science. 


\subsubsection{Relation to other non-linear phenomena}

The directional emission of light and atoms described in the previous sections is analogous to the superradiance discussed by Dicke [49]. He considered an elongated radiating system of incoherently electronically excited atoms, and showed that this system realizes "a laser which does not employ mirrors in order to produce feedback amplification." The amplification of this "Coherence brightened laser" is provided by electronic coherence: "The memory of the previously emitted electromagnetic field is burned into the radiating system rather than being sent back into the radiating system by the use of mirrors [49]." The key feature of superradiance (or superfluorescence) [54 58] is that spontaneous emission is not a single-atom process, but a collective process of all atoms, leaving the atoms in a coherent superposition of ground and excited states [59]. The condensate at rest "pumped" by the off-resonant laser corresponds to the electronically excited state in the Dicke case. It can decay by a spontaneous Raman process to a state with photon recoil (corresponding to the ground state). The rate of superradiant emission in Dicke's treatment is proportional to the square of an oscillating macroscopic dipole moment. In the present case, the radiated intensity is proportional to the square of the contrast of the matter wave interference pattern between the condensate and the recoiling atoms. In both cases, the initial emission of light shows the single atom dipole pattern. Quantum noise and spontaneous emission create spatial coherence and lead to directional emission different from forward scattering.

The situation of an atom cloud with a small excited state admixture ("dressed" condensate) nicely demonstrates the analogy between the optical laser and the atom laser. If the emitted light is allowed to build up in a cavity, an optical laser is realized (called the Coherent Atomic Recoil Laser (CARL) 60 62. If the matter wave field builds up in a cavity, it realizes an atom laser by optical pumping 63 65]. In our experiment, the matter wave field builds up in a traveling wave even without a cavity because of the slow motion of the recoiling atoms.

For smaller momentum transfer, the nature of the quasi-particles in the condensate will change from recoiling atoms to phonons. The observed phenomenon is therefore related to stimulated Brillouin scattering which was called "phonon maser action" [66] due to the amplification of sound waves. However, the analogy is closest when the roles of light and atoms are exchanged. In Brillouin scattering, the optical field builds up, whereas the phonon field generated by the momentum transfer is usually rapidly damped and adiabatically eliminated from the equations. In our case, the matter wave field builds up and the optical field can be eliminated due to the fact that light propagates at a velocity which is ten orders of magnitude faster than the atomic recoil velocity.

The observed phenomenon differs from other stimulated processes like Brillouin scattering and Dicke superradiance in two regards. In those cases, the electromagnetic field is treated either classically, or spontaneous emission ("quantum noise") is needed only to initiate the process, which then evolves classically after many photons have been accumulated. In our case, all of the light is emitted spontaneously, i.e. without optical stimulation. Furthermore, our system gives rise to a "cascade" of superradiant scattering processes which does not exist in the two-level superradiance systems studied so far.

More generally, one can regard the observed superradiance as an instability against spatial pattern formation triggered by noise. The spatial pattern is the periodic density modulation, the noise is the quantum noise which leads to spontaneous scattering. Spontaneous pattern formation is an interesting non-linear phenomenon studied in fluid mechanics, optics and chemical reactions [67].

Superradiance is based on the coherence of the emitting system, but it does not require quantum degeneracy. The condition for superradiance is that the gain exceed the losses, or that the superradiant decay time be shorter than any decoherence time. Above the BEC transition temperature $T_{c}$, thermal Doppler broadening results in a thirty times shorter decoherence time than for a condensate. Further, the larger size of the thermal cloud reduces the solid angle $\Omega_{j}$ and therefore the gain by another factor of ten. Therefore, the threshold for superradiance in a thermal cloud is several orders of magnitude higher than for a condensate. No signs of superradiant scattering were observed above $T_{c}$; rather, the sudden appearance of superradiant emission was a sensitive indicator for reaching the phase transition. 

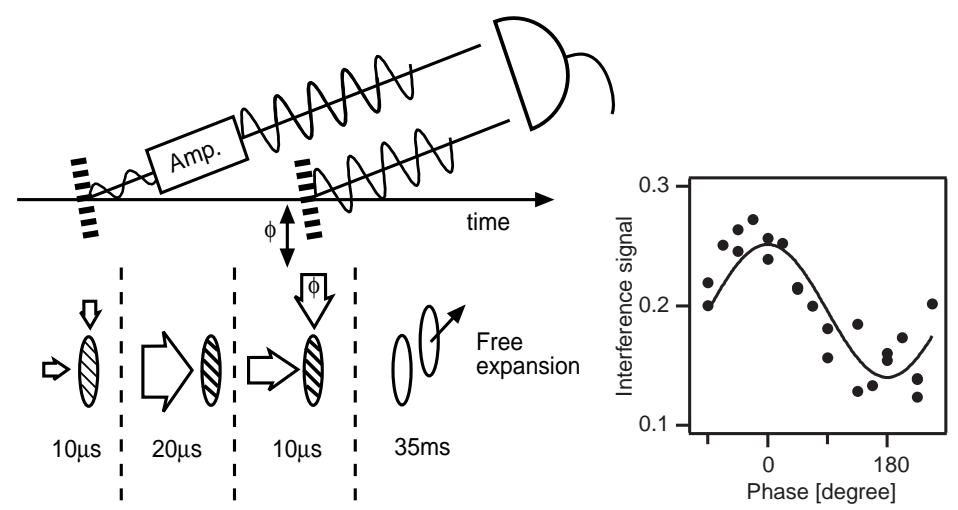

Fig. 15. Experimental scheme for observing phase coherent matter wave amplification. A small-amplitude matter wave was split off the condensate by applying a pulse of two off-resonant laser beams (Bragg pulse). This input matter wave was amplified by passing it through the condensate pumped by a laser beam. The coherence of the amplified wave was verified by observing its interference with a reference matter wave, which was produced by applying a second (reference) Bragg pulse to the condensate. The interference signal was observed after $35 \mathrm{~ms}$ of ballistic expansion. The fringes on the right side show the interference between the amplified input and the reference matter wave. Reprinted by permission from Nature, Ref. 68], copyright 1999 Macmillan Magazines Ltd.

\subsection{Phase-coherent amplification of matter waves}

Atom amplification differs from light amplification in one important aspect. Since the total number of atoms is conserved (in contrast to photons), the active medium of a matter wave amplifier has to include a reservoir of atoms. One also needs a coupling mechanism which transfers atoms from the reservoir to an input mode while conserving energy and momentum. The gain mechanism which was explained above can act as a matter wave amplifier. The momentum required to transfer atoms from the condensate at rest to the input mode is provided by light scattering. Refs. [47, 50] discussed that a condensate pumped by an off-resonant laser beam acts as a matter wave amplifier which can amplify input matter waves within the momentum range which can be reached by scattering a single pump photon.

The inversion in this matter wave amplifier is most apparent in the dressed atom picture where the condensate at rest and the pump light field are treated as one system. An atom in the dressed condensate can now spontaneously decay into a recoiling atom and a scattered photon which escapes. Inversion is maintained since the photons escape and thus the inverse process of combining recoiling atom and emitted photon into a dressed atom at rest is not possible. Thus, in principle, a complete transfer of the condensate atoms into the recoil mode can occur.

Our observation of superradiance can be regarded as the observation of matter wave amplification of noise, i.e. of spontaneously scattered atoms. To examine this amplification mechanism further, we performed an experiment in which, rather than examine the amplification of noise, we amplify a well-defined input signal, i.e. a matter wave of well-defined momentum which was generated by Bragg scattering. By comparing the input and output waves, we could characterize the amplification process.

The input atoms were generated by exposing the condensate to a pulsed optical standing wave which transferred a small fraction of the atoms (between $10^{-4}$ and $10^{-2}$ ) into a recoil mode by Bragg diffraction [10,15]. Both laser beams were red-detuned by $1.7 \mathrm{GHz}$ from the $3 S_{1 / 2},|F=1\rangle \rightarrow$ $3 P_{3 / 2},|F=0,1,2\rangle$ transition to suppress normal Rayleigh scattering. The geometry of the light beams is shown in Fig. 15. The beam which was perpendicular to the long axis of the condensate (radial beam) was blue detuned by $50 \mathrm{kHz}$ relative to the axial beam. This detuning fulfilled the Bragg resonance condition. 


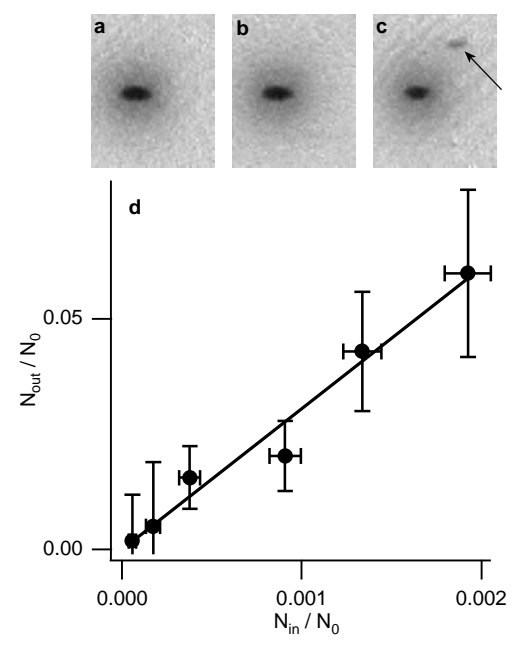

Fig. 16. Input-output characteristic of the matter-wave amplifier. (a-c) Typical time-of-flight absorption images demonstrating matter wave amplification. The output of the seeded amplifier (c) is clearly visible, whereas no recoiling atoms are discernible in the case without amplification (a) or amplification without the input (b). The size of the images is $2.8 \mathrm{~mm} \times 2.3 \mathrm{~mm}$. (d) Output of the amplifier as a function of the number of atoms at the input. A straight line fit shows a number gain of 30. Reprinted by permission from Nature, Ref. 68, copyright 1999 Macmillan Magazines Ltd.

Amplification of the input matter wave was realized by applying an intense radial pump pulse for the next $20 \mu$ s with a typical intensity of $40 \mathrm{~mW} / \mathrm{cm}^{2}$. The number of atoms in the recoil mode was determined by suddenly switching off the trap and observing the ballistically expanding atoms after $35 \mathrm{~ms}$ of time-of-flight using resonant absorption imaging. After the expansion, the condensate and the recoiling atoms were fully separated (Fig. 16ic).

Fig. 16 shows the input-output characteristics of the amplifier. The number of input atoms was below the detection limit of our absorption imaging (Fig. 16a) and was determined from a calibration of the Bragg process at high laser powers, where the diffracted atoms were clearly visible in the images. The amplification pulse alone, although above the threshold for superradiance [32], did not generate a discernible signal of atoms in the recoil mode (Fig. 16b). When the weak input matter wave was added, the amplified signal was clearly visible (Fig. 16ic). The gain was controlled by the intensity of the pump pulse (see Eq. (3.11)) and typically varied between 10 and 100. Fig. 16d shows the observed linear relationship between the atom numbers in the input and the amplified output with a number gain of 30 .

The phase of the amplified matter wave was determined with an interferometric technique (Fig. 15). For this, a reference matter wave was split off the condensate in the same way as the first (input) wave. The phase of the reference matter wave was scanned by shifting the phase of the radio-frequency signal that drove the acousto-optic modulator generating the axial Bragg beam. We then observed the interference between the reference and the amplified matter waves by measuring the number of atoms in the recoil mode.

When the input was comparable in intensity to the reference matter wave, high contrast fringes were observed even without amplification. Fringes were barely visible when the input was about 40 times weaker in population. After amplification, we regained a large visibility (Fig. 15). This increase in visibility proved the coherent nature of the matter wave amplification process. The increase in visibility of the interference fringes was a factor of two, less than the expected square root of the total gain of thirty. This might be due to a distortion of the matter wave during the amplification, but this effect requires further study. A similar experiment with rubidium atoms was done at the University of Tokyo [69]. 
This experiment can be regarded as a demonstration of an active atom interferometer. It realizes a two-pulse atom interferometer with phase-coherent amplification in one of the arms. Such active interferometers may be advantageous for precise measurements of phase shifts in highly absorptive media, e.g. for measurements of the index of (matter wave) refraction when a condensate passes through a gas of atoms or molecules 77 . Since the most accurate optical gyroscopes are active interferometers [71, atom amplification might also play a role in future matter-wave gyroscopes [72].

\section{Spinor Bose-Einstein condensates}

The experiments described in the previous sections explored the nature of Bose-Einstein condensates of atomic gases in which all the atoms were in the same internal state, in particular, the $\left|F=1, m_{F}=-1\right\rangle$ hyperfine state of sodium. The Bose-Einstein condensation phase transition leads to a non-zero value of a scalar order parameter, the condensate wavefunction $\psi(\vec{r})=\langle\hat{\Psi}(\vec{r})\rangle$. As such, a single-component gaseous condensate can be considered as a simple, tractable model of the more complicated spinless superfluid ${ }^{4} \mathrm{He}$. Many experiments in recent years have explored aspects of this connection between the two quantum fluids [1].

However, unlike ${ }^{4} \mathrm{He}$, alkali atoms can have a non-zero spin and therefore numerous internal hyperfine states which are stable electronic ground states. Thus, there exists the possibility of creating a quantum fluid simultaneously composed of several, distinguishable components by Bose condensing a gas of atoms in several hyperfine states.

The study of multi-component superfluid systems has been a tantalizing goal of low-temperature physics for decades. The earliest discussion focused on ${ }^{4} \mathrm{He}-{ }^{6} \mathrm{He}$ mixtures. ${ }^{6} \mathrm{He}$ is radioactive with a half-life of 1 second. An ambitious experiment by Guttman and Arnold [73 in 1953 sought evidence for the superfluid flow of ${ }^{6} \mathrm{He}$ mixed with ${ }^{4} \mathrm{He}$ to no avail. Nevertheless, this pursuit touched off a series of theoretical works on two-component superfluid hydrodynamics [74, and others since]. In 1978, Colson and Fetter [75] considered such mixtures in the context of mean-field theories which apply directly to current experiments, and discussed the criterion for interactions between the superfluids to cause miscibility or phase-separation. After progress in the stabilization of a spin-polarized atomic hydrogen gas, Siggia and Ruckenstein [76 considered the use of different hyperfine states to achieve a mixture of superfluids. Since the observation of gaseous Bose condensates, the interest in multi-component condensates has been revived with a flurry of theoretical attention [77 80, for example].

For atomic gases in magnetic traps, the availability of hyperfine states is restricted by the requirement that the trapped atoms remain in weak-field seeking states. For example, alkali atoms with a nuclear spin of $I=3 / 2$, such as ${ }^{87} \mathrm{Rb}$ and ${ }^{23} \mathrm{Na}$, have three weak-field seeking states at zero-field: one in the lower hyperfine manifold $\left(\left|F=1, m_{F}=-1\right\rangle\right)$, and two in the upper hyperfine manifold $\left(\left|F=2, m_{F}=1,2\right\rangle\right)$. Generally, the simultaneous confinement of more than one of these states is unstable against exothermic hyperfine state changing collisions.

However, it was recently found that magnetically-trapped multi-component gases of ${ }^{87} \mathrm{Rb}$ are quite long lived due to a fortunate near-equality of the singlet and triplet scattering lengths which greatly suppresses the spin exchange collision rate 81 84. This allowed for the creation of multi-component condensates by the simultaneous magnetic confinement of ${ }^{87} \mathrm{Rb}$ atoms in the $\left|F=1, m_{F}=-1\right\rangle$ and $\left|F=2, m_{F}=2\right\rangle$ [81] (and, in later work, $\left|F=2, m_{F}=1\right\rangle$ ) states. The Boulder group has used magnetically trapped multi-component condensates for a remarkable series of experiments. Studies have probed the spatial separation of a two-component Bose-Einstein condensate 81, 85] and the stability of a relative phase between the two components even in the presence of dissipation [86. The Boulder group has also explored the nature of multi-component condensates in the presence of continuous resonant and non-resonant rf coupling between the components. Such coupling links the external center-of-mass and the internal degrees of freedom of the condensed gas and gives rise to a rich variety of dynamical effects such as Josephson-type oscillations 87] and spin-wave excitations 88, 89, as well as new techniques for manipulating the phase of the condensate wavefunction [90, 91]. Some of this work is reviewed in Refs. 92, 93.

In contrast, a far-off-resonant optical trap confines atoms regardless of their hyperfine state 44. Thus, the atomic spin is liberated from the requirements of magnetic trapping and becomes a new 
degree of freedom. In particular, all atoms in the lower hyperfine manifold, for example the $F=1$ hyperfine manifold of sodium, can be stably trapped simultaneously without suffering from hyperfine manifold changing collisions. Such multi-component optically trapped condensates are represented by an order parameter which is a vector in hyperfine spin space, and are thus called spinor Bose-Einstein condensates. A variety of new phenomena are predicted for this new quantum fluid such as spin textures, spin waves, and coupling between atomic spin and superfluid flow [94 96].

Spinor Bose condensates differ from other multi-component Bose condensates, such as the experimentally realized ${ }^{87} \mathrm{Rb}$ mixtures or the proposed mixtures of several atomic species, in important ways stemming from symmetries under rotations of the vectorial order parameter. Furthermore, spin relaxation collisions within the lower $F=1$ hyperfine manifold

$$
\left|m_{\mathrm{F}}=0\right\rangle+\left|m_{\mathrm{F}}=0\right\rangle \leftrightarrow\left|m_{\mathrm{F}}=+1\right\rangle+\left|m_{\mathrm{F}}=-1\right\rangle
$$

allow for population mixing among the different hyperfine states without trap loss. In contrast, spin relaxation is the major limitation to the lifetime (about a second) of the ${ }^{87} \mathrm{Rb}$ mixtures.

Since the realization of an optical trap for Bose-Einstein condensates 44], our group has performed several experimental studies of this new quantum fluid. In three different experiments we explored the ground-state spin structure of spinor condensates in external magnetic fields 97, the formation and persistence of metastable spin domain configurations [98], and the transport across spin domain boundaries by quantum tunneling [99]. In this section, we summarize our current understanding of this fluid as derived from our experiments and from a growing number of theoretical works. While a portion of this work has been reviewed in Refs. [1, 100], this section is the first comprehensive review on spinor Bose-Einstein condensates.

\subsection{The implications of rotational symmetry}

An $F=1$ spinor Bose-Einstein condensate is described by a three-component order parameter

$$
\vec{\psi}(\vec{r})=\left(\begin{array}{c}
\psi_{1}(\vec{r}) \\
\psi_{0}(\vec{r}) \\
\psi_{-1}(\vec{r})
\end{array}\right)
$$

and can thus be regarded as a particular instance of a multi-component condensate. Here, the subscripts refer to the spin projection on the quantization axis, $m_{F}=-1,0,+1$. However, the spinor Bose-Einstein condensate is distinguished from a general, multi-component quantum fluid by the fact that the order parameter $\vec{\psi}$ transforms as a vector. The vectorial character of the order parameter has a pronounced effect on interatomic interactions, and defines important features of the spinor condensate at zero magnetic field, where the rotational symmetry of the system is preserved.

In second-quantized notation, the Hamiltonian for a multi-component gas has the general form

$$
\begin{aligned}
\hat{\mathcal{H}}= & \int d^{3} \vec{r}\left\{\hat{\Psi}_{i}^{\dagger}(\vec{r})\left(-\frac{\hbar^{2} \nabla^{2}}{2 m} \delta_{i j}+U_{i j}(\vec{r})\right) \hat{\Psi}_{j}(\vec{r})\right. \\
& \left.+\frac{g_{i j, k l}}{2} \int d^{3} \vec{r}_{1} d^{3} \vec{r}_{2} \hat{\Psi}_{i}^{\dagger}\left(\vec{r}_{1}\right) \hat{\Psi}_{j}^{\dagger}\left(\vec{r}_{2}\right) \hat{\Psi}_{k}\left(\vec{r}_{2}\right) \hat{\Psi}_{l}\left(\vec{r}_{1}\right) \delta\left(\vec{r}_{1}-\vec{r}_{2}\right)\right\}
\end{aligned}
$$

where the indices $i, j, k, l$ correspond to the $N$ components of the gas, and repeated indices are summed. The general form of the external potential $U_{i j}(\vec{r})$ allows for a potential which is not diagonal in the hyperfine spin basis, in which case it can represent a Josephson-type coupling between spin components. The $U_{i j}(\vec{r})$ terms also contain the effects of magnetic fields which are discussed in later sections.

The interatomic interaction (second line of the above expression) has been approximated as a contact interaction in which the coefficients $g_{i j, k l}$ describe the strength of the various elastic and inelastic (state converting) collisions. These generally constitute a large number of free parameters. Particle exchange $\left(g_{i j, k l}=g_{j i, k l}\right)$ and time-reversal $\left(g_{i j, k l}=g_{k l, i j}\right)$ symmetries reduce the number 
of free parameters from $N^{4}$ to $\left[\left(N^{2}+N+2\right)\left(N^{2}+N\right)\right] / 8$ but still a large number of interaction parameters remain 3 . For a three-component condensate, the number of interaction parameters is 21.

This situation is greatly simplified in the case of spinor condensates due to rotational symmetry. The rotationally symmetric characterization of two-body collisions among atoms of hyperfine spin $F_{1}$ and $F_{2}$ can only depend on their total spin $f=F_{1}+F_{2}$ and not on its orientation. Thus, in the $s$-wave limit, the interatomic interaction $V_{\text {int }}\left(\vec{r}_{1}-\vec{r}_{2}\right)$ is reduced to the form [94, 95]

$$
V_{\mathrm{int}}\left(\vec{r}_{1}-\vec{r}_{2}\right)=\frac{4 \pi \hbar^{2}}{m} \delta\left(\vec{r}_{1}-\vec{r}_{2}\right) \sum_{f} a_{f} \tilde{\mathcal{P}}_{f}
$$

where $a_{f}$ is the scattering length for collisions between atoms with total spin $f$, and $\tilde{\mathcal{P}}_{f}$ is the projection operator for the total spin. For colliding bosons of spin $F, f$ can take the values $f=0,2, \ldots, 2 F$. Therefore, the interaction parameters $g_{i j, k l}$ describing collisions among the $N=2 F+1$ different components of a spinor condensate of spin $F$ depend only on $F+1=(N+1) / 2$ scattering lengths.

In particular, for the $F=1$ spinor system which was realized in gaseous sodium, interactions are described fully by just two parameters, the scattering lengths $a_{f=0}$ and $a_{f=2}$. The interaction potential can then be written as

$$
V_{\text {int }}\left(\vec{r}_{1}-\vec{r}_{2}\right)=\left(g_{0}+g_{2} \vec{F}_{1} \cdot \vec{F}_{2}\right) \delta\left(\vec{r}_{1}-\vec{r}_{2}\right)
$$

where the parameters $g_{0}$ and $g_{2}$ are defined as

$$
\begin{aligned}
& g_{0}=\frac{4 \pi \hbar^{2}}{m} \frac{2 a_{f=2}+a_{f=0}}{3} \\
& g_{2}=\frac{4 \pi \hbar^{2}}{m} \frac{a_{f=2}-a_{f=0}}{3}
\end{aligned}
$$

and $\vec{F}_{1}$ and $\vec{F}_{2}$ are the spin operators for the two colliding particles. The spin-dependence of the interatomic interaction is thereby isolated in the rotationally-symmetric term $g_{2} \vec{F}_{1} \cdot \vec{F}_{2} \delta\left(\vec{r}_{1}-\vec{r}_{2}\right)$. The Hamiltonian describing a weakly-interacting Bose gas of spin $F=1$ now becomes 94, 95,

$$
\begin{aligned}
\hat{\mathcal{H}}= & \int d^{3} \vec{r}\left\{\hat{\Psi}_{i}^{\dagger}(\vec{r})\left(-\frac{\hbar^{2} \nabla^{2}}{2 m} \delta_{i j}+V_{i j}(\vec{r})\right) \hat{\Psi}_{j}(\vec{r})\right. \\
& +\frac{1}{2}\left[g_{0} \hat{\Psi}_{i}^{\dagger}(\vec{r}) \hat{\Psi}_{j}^{\dagger}(\vec{r}) \hat{\Psi}_{i}(\vec{r}) \hat{\Psi}_{j}(\vec{r})\right. \\
& \left.\left.+g_{2}\left(\hat{\Psi}_{i}^{\dagger}(\vec{r})\left(F_{\eta}\right)_{i j} \hat{\Psi}_{j}(\vec{r})\right) \cdot\left(\hat{\Psi}_{k}^{\dagger}(\vec{r})\left(F_{\eta}\right)_{k l} \hat{\Psi}_{l}(\vec{r})\right)\right]\right\}
\end{aligned}
$$

where the index $\eta$ runs over the three coordinate axes $x, y, z$.

Equivalently, the Hamiltonian can be written in the form of Eq. 4.3, where all non-zero $g_{i j, k l}$ are determined by $g_{0}$ and $g_{2}$ as shown in Table 3 . Taking into account particle exchange symmetries and gathering similar terms, the interaction Hamiltonian can be written as

$$
\begin{aligned}
\mathcal{H}_{\mathrm{int}}= & \frac{1}{2} \int d^{3} \vec{r}\left[\left(g_{0}+g_{2}\right) \hat{\Psi}_{1}^{\dagger} \hat{\Psi}_{1}^{\dagger} \hat{\Psi}_{1} \hat{\Psi}_{1}+g_{0} \hat{\Psi}_{0}^{\dagger} \hat{\Psi}_{0}^{\dagger} \hat{\Psi}_{0} \hat{\Psi}_{0}\right. \\
& +\left(g_{0}+g_{2}\right) \hat{\Psi}_{-1}^{\dagger} \hat{\Psi}_{-1}^{\dagger} \hat{\Psi}_{-1} \hat{\Psi}_{-1}+2\left(g_{0}+g_{2}\right) \hat{\Psi}_{1}^{\dagger} \hat{\Psi}_{0}^{\dagger} \hat{\Psi}_{1} \hat{\Psi}_{0} \\
& +2\left(g_{0}+g_{2}\right) \hat{\Psi}_{-1}^{\dagger} \hat{\Psi}_{0}^{\dagger} \hat{\Psi}_{-1} \hat{\Psi}_{0}+2\left(g_{0}-g_{2}\right) \hat{\Psi}_{1}^{\dagger} \hat{\Psi}_{-1}^{\dagger} \hat{\Psi}_{1} \hat{\Psi}_{-1} \\
& \left.+2 g_{2}\left(\hat{\Psi}_{0}^{\dagger} \hat{\Psi}_{0}^{\dagger} \hat{\Psi}_{1} \hat{\Psi}_{-1}+\hat{\Psi}_{1}^{\dagger} \hat{\Psi}_{-1}^{\dagger} \hat{\Psi}_{0} \hat{\Psi}_{0}\right)\right]
\end{aligned}
$$

The first three terms are the self-scattering terms, the following three the cross-scattering terms, and the last line contains the spin relaxation terms.

\footnotetext{
${ }^{3}$ Due to particle exchange symmetry, we may denote the interaction parameter as $g_{\mathcal{A}, \mathcal{B}}$ where $\mathcal{A}$ and $\mathcal{B}$ are elements of the set $\mathbb{P}$ of distinct unordered pairs of indices $i, j$. There are $Z=N(N+1) / 2$ such pairs. Due to time-reversal symmetry, the interaction parameters are enumerated as $g_{\mathcal{Z}}$ where $\mathcal{Z}$ is an unordered pair of elements from $\mathbb{P}$. Thus, the number of free interaction parameters is $Z(Z+1) / 2=\left[\left(N^{2}+N+2\right)\left(N^{2}+N\right)\right] / 8$.
} 


\begin{tabular}{c|c|c|c}
$g_{A B, A B}$ & $m_{F}=+1$ & $m_{F}=0$ & $m_{F}=-1$ \\
\hline$m_{F}=+1$ & $g_{0}+g_{2}$ & $\frac{g_{0}+g_{2}}{2}$ & $\frac{g_{0}-g_{2}}{2}$ \\
$m_{F}=0$ & $\frac{g_{0}+g_{2}}{g_{0}}$ & $\frac{g_{0}+g_{2}}{2}$ \\
$m_{F}=-1$ & $\frac{g_{0}-g_{2}}{2}$ & $\frac{g_{0}+g_{2}}{2}$ & $g_{0}+g_{2}$
\end{tabular}

Table 3. Interaction parameters $g_{i j, k l}$ describing elastic collisions in the $F=1$ spinor system. The interaction Hamiltonian is defined as in Eq.4.3. The interaction parameter responsible for spin relaxation is $g_{00,-1+1}=g_{2}$. The cross-species scattering length discussed in Sec. 4.6 is given as $g_{A B, A B}=2 \pi \hbar^{2} a_{A B} / \mathrm{m}$.

It is important to note that this simplification of the interaction term is only strictly valid in the absence of magnetic fields. As the magnetic field is increased, the hyperfine spin $F$ is no longer a good quantum number for describing the atomic states, and thus collisional properties deviate from the relations imposed by rotational symmetry. Dramatic deviations from rotational symmetry can be seen, for example, at a Feshbach resonance where the scattering length for one particular collisional channel is greatly varied. Nonetheless, away from Feshbach resonances and at low magnetic fields for which the Zeeman shifts are much smaller than the hyperfine splitting, one can expect the zero-field description of collisional interactions to remain valid.

The properties of spinor Bose-Einstein condensates at zero-magnetic field have been recently discussed by a number of authors. Ho [94] and Ohmi and Machida 95] considered the ground state of a spinor condensate by an extension of mean-field theory (see also 101]). The $N$-particle condensate ground state is found by replacing the field operators $\hat{\Psi}_{i}$ in Eq. 4.8 with $c$-number order parameters $\psi_{i}$. It is convenient to express the order parameter as $\vec{\psi}=\sqrt{n} \vec{\zeta}$ where $n$ is the atomic density and $\vec{\zeta}$ is a three-component spinor of normalization $|\vec{\zeta}|=1$, obtaining the energy functional

$$
E=\int d^{3} \vec{r}\left[\psi_{i}^{*}(\vec{r})\left(-\frac{\hbar^{2} \nabla^{2}}{2 m}\right) \psi_{i}(\vec{r})+(U(\vec{r})-\mu) n(\vec{r})+\frac{n^{2}}{2}\left(g_{0}+g_{2}\langle\vec{F}\rangle^{2}\right)\right]
$$

Here it is assumed that the external potential is scalar, i.e. diagonal in the hyperfine spin basis and equal for each of the spin components. The chemical potential $\mu$ determines the number of atoms in the condensate.

The ground-state spinor $\vec{\zeta}$ is determined by minimizing the spin-dependent interaction energy, $n^{2} g_{2}\langle\vec{F}\rangle^{2} / 2$, which is expressed in terms of the average condensate spin $\langle\vec{F}\rangle_{\eta}=\zeta_{i}^{*}\left(F_{\eta}\right)_{i j} \zeta_{j}$. There are two distinct solutions depending on the sign of the spin-dependent interaction parameter $g_{2}$ :

- $g_{2}>0$ : the collisional coupling is anti-ferromagnetic as the condensate lowers its energy by minimizing its average spin, i.e. by making $|\langle\vec{F}\rangle|=0$. The ground state spinor is then one of a degenerate set of spinors, the "polar" states, corresponding to all possible rotations of the hyperfine state $\left|m_{F}=0\right\rangle$.

- $g_{2}<0$ : the collisional coupling is ferromagnetic as the condensate lowers its energy by maximizing its average spin, i.e. by making $|\langle\vec{F}\rangle|=1$. In this case the ground state spinors correspond to all rotations of the hyperfine state $\left|m_{F}=+1\right\rangle$.

Law, $\mathrm{Pu}$, and Bigelow [96] explicitly calculated the many-body state of a homogeneous spinor Bose-Einstein condensate without assuming a Hartree form. They describe an elegant transformation of the spinor Hamiltonian in terms of operators which obey an angular momentum algebra, and thus immediately yield eigenstates of the many-body Hamiltonian and their energy spectrum. Antiferromagnetic coupling leads to a unique ground-state which cannot be represented in the Hartree form and which has super-Poissonian fluctuations in the population of each spin state. Ferromagnetic coupling yields a degenerate family of ground states with sub-Poissonian fluctuations. The Hartree solution with all atoms in the $\left|m_{F}=+1\right\rangle$ state is included in the family of ferromagnetic ground states obtained by Law, $\mathrm{Pu}$ and Bigelow. 


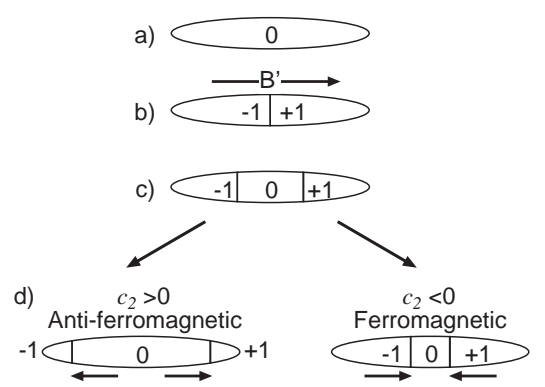

Fig. 17. Constructing the ground-state $\left\langle F_{z}\right\rangle=0$ spinor in a trap. In each step $(\mathrm{a}-\mathrm{d})$, another contribution to the Hamiltonian is considered (see text): a) linear Zeeman shift from homogeneous field, b) linear Zeeman shift from field gradient, c) quadratic Zeeman shift, d) spin-dependent mean-field interaction.

The highly entangled ground state of Law et al. for the anti-ferromagnetic case differs in energy from the ground state in the mean-field formalism (all atoms in the $\left|m_{F}=0\right\rangle$ state) only by a term which is proportional to $N$, the number of atoms. This energy is $N$ times smaller than the meanfield energy which scales as $N^{2}$. Therefore, it will be difficult to observe this highly correlated state. Several authors have extended the treatment to finite magnetic fields and shown that the singlet state of Ref. [96] is only the ground state at very small magnetic fields; otherwise the ground state approaches the Hartree form [102,103].

The scattering lengths $a_{f=0}$ and $a_{f=2}$ can be calculated by incorporating data from a variety of experiments into models of the interparticle potentials. For sodium, it is predicted that $a_{f=2}=2.75$ $\mathrm{nm}$ 104 and $a_{f=2}-a_{f=0}=0.29 \mathrm{~nm}$ 105]. Thus, an $F=1$ spinor condensate of sodium should be anti-ferromagnetic. For ${ }^{87} \mathrm{Rb}$, it appears that $a_{f=2}-a_{f=0}<0$ and thus an $F=1$ spinor condensate of ${ }^{87} \mathrm{Rb}$ should be ferromagnetic [94].

\subsection{Tailoring the ground-state structure with magnetic fields}

These theoretical considerations differ from the conditions in our experiment in two major regards: the imposition of spin conservation, and the effects of field inhomogeneities and quadratic Zeeman shifts. These differences are exemplified by considering the outcome of an experiment we performed, in which an optically-trapped Bose condensed cloud was prepared with all atoms in the $\left|m_{F}=0\right\rangle$ state. The cloud was then allowed to equilibrate to the ground state by spin relaxation and by spatial redistribution 97. For simplicity, consider only variations in the condensate wavefunction in one dimension, the $\hat{z}$-direction, and define the coordinate $z$ to give the distance from the center of the condensate. This corresponds to the experimental situation in which the spinor condensates were held in the highly anisotropic, cigar-shaped potential of an optical trap.

Let us consider the evolution of the pure $m_{F}=0$ condensate as we slowly add the effects of magnetic fields and mean-field interactions and construct the relevant energy functional (Fig. 17).

a) Linear Zeeman shift from homogeneous field. In a homogeneous magnetic field of strength $B_{0}$, the linear Zeeman energy

$$
E_{\text {lin }}=-p \int d^{3} r n\left\langle F_{z}\right\rangle
$$

is minimized by placing all atoms in the strong-field seeking $\left|m_{F}=+1\right\rangle$ state (a ferromagnetic state) Here, $p=g \mu_{B} B_{0}$ where $g$ is the Landé $g$-factor and $\mu_{B}$ is the Bohr magneton.

However, spin non-conserving collisions (dipolar relaxation), which would be necessary to transform the $m_{F}=0$ condensate to the $m_{F}=+1$ ground state, are negligible over the lifetime of the

\footnotetext{
4 The operator $F_{z}$ gives the component of the spin along the direction of the magnetic field. This direction need not be the same as the long axis of the trapped condensate.
} 
condensate. Thus, the total spin is a conserved quantity, and rather than considering the global ground state of the system, one must consider the lowest energy state under the restriction of spin conservation. Formally, one minimizes the restricted energy functional

$$
K_{\text {tot }}=E_{\text {tot }}+\tilde{p} \int d^{3} \vec{r} n\left\langle F_{z}\right\rangle
$$

where $\tilde{p}$ is a Lagrange parameter determined by the given value of the total spin. For the case where $\int\left\langle F_{z}\right\rangle n d^{3} \vec{r}=0$, the linear Zeeman shift of a homogeneous magnetic field $\left(E_{l Z}\right)$ is exactly canceled $\left(p_{0}=\tilde{p}\right)$.

This is an important point: spin conservation allows one to study the effects of the small spin-dependent interaction energies even at magnetic fields for which the linear Zeeman energy would otherwise be dominant. For example, in the case of sodium, the spin-dependent meanfield energy is just $c_{2} n \simeq h \times 50 \mathrm{~Hz}$ for a typical density of $n=3 \times 10^{14} \mathrm{~cm}^{-3}$. Thus, without the restriction of spin conservation, the ground-state spinor would trivially consist of all atoms in the $\left|m_{F}=+1\right\rangle$ state at a magnetic field of just $70 \mu \mathrm{G}$, and any interesting structure, correlations or dynamics due to the anti-ferromagnetic coupling would be obscured. However, due to spin conservation, many of these effects can be studied even in the absence of such demanding field stability.

b) Linear Zeeman shift from field gradient. A field gradient $B^{\prime}$ along the long axis of the condensate introduces an energy term

$$
E_{\text {grad }}=-\int d^{3} \vec{r} p(z) n\left\langle F_{z}\right\rangle
$$

with $p(z)=g \mu_{B} B^{\prime} z$, which makes it energetically favorable for two $m_{F}=0$ atoms to collide and produce a $m_{F}=+1$ atom on the high-field end of the cloud, and a $m_{F}=-1$ atom on the low-field end. Thus, the condensate is magnetically polarized into two pure spin domains.

c) Quadratic Zeeman shift from homogeneous field. The quadratic Zeeman shift at a field $B_{0}$ introduces an energy term of the form

$$
E_{\text {quad }}=q \int d^{3} \vec{r} n\left\langle F_{z}^{2}\right\rangle
$$

which causes the energy of a $m_{F}=0$ atom to be lower than the average energy of a $m_{F}=+1$ and $m_{F}=-1$ atom by an amount $q=\hat{q} B_{0}^{2}$. For sodium, $\hat{q}=h \times 390 \mathrm{~Hz} / \mathrm{G}^{2}$. The quadratic Zeeman energy favors population in the $\left|m_{F}=0\right\rangle$ state, and thus introduces an $m_{F}=0$ domain at the center of the cloud with boundaries at $q=|p(z)|$ (see Fig. 17. ).

d) Spin-dependent mean-field interaction. As discussed previously, the collisional interactions give a spin-dependent energy term of the form

$$
E_{\text {int }}=\frac{1}{2} \int d^{3} \vec{r} g_{2} n^{2}\langle\vec{F}\rangle^{2}
$$

Anti-ferromagnetic coupling $\left(g_{2}>0\right)$ favors the polar state, and thus makes the central $m_{F}=0$ domain larger. Ferromagnetic coupling $\left(g_{2}<0\right)$ favors the ferromagnetic states $\left|m_{f}=+1\right\rangle$ and $\left|m_{F}=-1\right\rangle$ at the ends of the cloud, and thus the $m_{F}=0$ domain shrinks (see Fig. $17 \mathrm{~d}$ ).

Collecting spin-dependent energy terms, we now have the restricted energy functional

$$
\begin{aligned}
K_{\mathrm{tot}} & =\int d^{3} \vec{r}\left(K_{0}+K_{\mathrm{spin}}\right) \\
K_{0} & =\vec{\psi}^{\dagger}\left(-\frac{\hbar^{2} \nabla^{2}}{2 m}\right) \vec{\psi}+\left(U(\vec{r})-\mu+\frac{g_{0}}{2} n\right) n \\
K_{\mathrm{spin}} & =\left(-p(z)\left\langle F_{z}\right\rangle+q\left\langle F_{z}^{2}\right\rangle+c\langle\vec{F}\rangle^{2}\right) n
\end{aligned}
$$

where $c=g_{2} n / 2$. The ground-state spin structure of a spinor condensate is found by minimizing $K_{\text {tot }}$. 

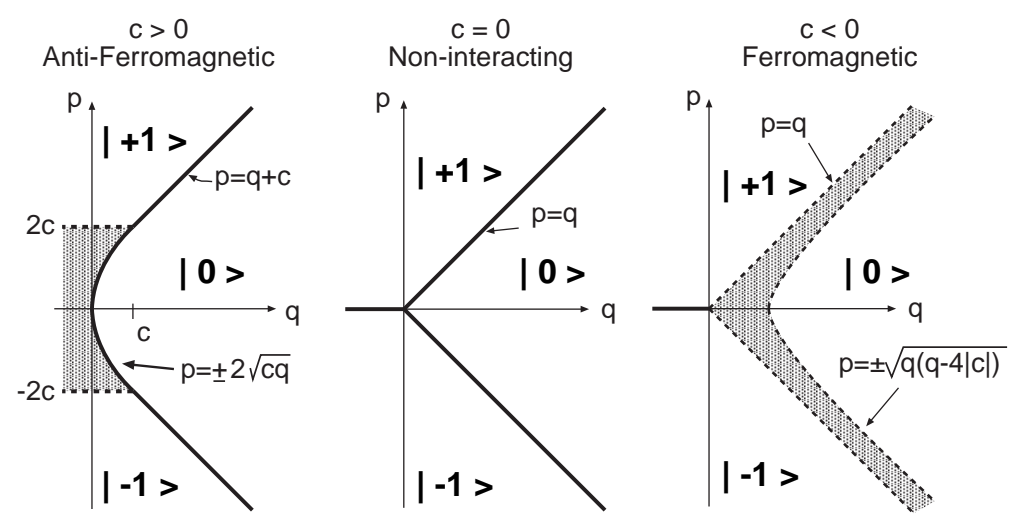

Fig. 18. Spin-domain diagrams for $F=1$ spinor condensates for the three cases $c>0$ (anti-ferromagnetic collisional coupling), $c=0$ (no spin-dependent interactions), and $c<0$ (ferromagnetic coupling). The ground-state spinors which minimize $K_{\text {spin }}$ for the given values of $p, q$, and $c$ are shown. Regions in which the ground state contains just one component are labeled by the magnetic hyperfine sublevel. Regions of mixed components are shaded. The ground-state spin-domain structure of a spinor condensate in the presence of a magnetic bias field and field gradient is indicated by a vertical line across the relevant domain diagram. Reprinted by permission from Nature, 97], copyright 1999 Macmillan Magazines Ltd.

\subsection{Spin-domain diagrams: a local density approximation to the spin structure of spinor condensates}

Focusing on the spin-dependent part, $K_{\text {spin }}$ has a simple and elegant form. Let us step away from the specific experimental considerations and consider a homogeneous spinor condensate with some arbitrary, uniform values of the parameters $p, q$ and $c$. The ground-state spinors obtained by minimizing the energy functional $K_{\text {spin }}$ are indicated in the three diagrams of Fig. 18 for three conditions on the parameter $c$ 97]. For a non-interacting gas $(c=0)$, the ground-state spinor is determined by the effects of magnetic fields alone.

In the case of anti-ferromagnetic coupling $(c>0)$, the spinor diagram changes in two significant ways. First, since the anti-ferromagnetic energy favors the $\left|m_{F}=0\right\rangle$ polar state, the region in which the $\left|m_{F}=0\right\rangle$ state is the ground state is enlarged. Second, at low values of $q$, in addition to the region of pure hyperfine states, a region is introduced in which the ground-state spinor is a superposition of the $\left|m_{F}= \pm 1\right\rangle$ states. While admixing the $\left|m_{F}=-1\right\rangle\left(\left|m_{F}=+1\right\rangle\right)$ state for $p>0(p<0)$ increases the linear Zeeman energy, it reduces the interaction energy by making the superposition state more polar in character. Explicitly, in the shaded region, $\left\langle F_{z}\right\rangle=p / 2 c$ independent of $q$. The effect of the linear Zeeman energy on anti-ferromagnetic condensates was considered also by Ohmi and Machida [95] whose work did not include the quadratic Zeeman energy, and thus concerned the $q=0$ axis of the spin-domain diagram.

In the case of ferromagnetic coupling $(c<0)$, the situation is different. The higher interaction energy for the polar state diminishes the region in which the ground state is the $\left|m_{F}=0\right\rangle$ state. In between the regions of single-component ground-state spinors there is a region in which all three hyperfine states are generally mixed. In these regions, the $\left|m_{F}=0\right\rangle$ state is mixed predominantly with a large population of only one of the $\left|m_{F}= \pm 1\right\rangle$ states and with a small population of the other.

Returning now to the experimental situation, the spin-domain diagram is used to describe the ground-state spin structure of a spinor condensate through a local density approximation. The values of $q$ and $c$ (but not its sign) are determined by the magnetic bias field and by the condensate density, which we assume for now to be constant across the condensate. The coefficient $p$ varies across the condensate due to the presence of a magnetic field gradient; thus, the variations in the condensate spin structure across the length of the condensate are determined by scanning along a vertical line in the spin-domain diagrams. The length of this line is determined by the condensate length and the field gradient $B^{\prime}$. The center of this vertical line is determined by the total spin in the condensate: it 


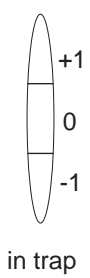

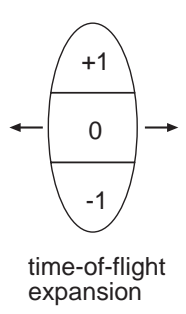
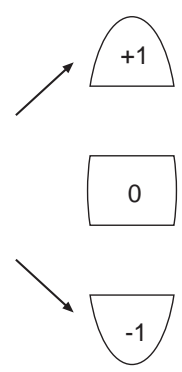

Stern-Gerlach

separation

Fig. 19. Probing spinor condensates. After release from the elongated optical trap, the trapped spinor condensate expands primarily radially while maintaining the axial hyperfine distribution. A magnetic field gradient is then used to separate out the different components while preserving their shape. A subsequent absorption probe reveals the spatial and hyperfine distributions in the trap.

moves upwards (larger $p$ ) as $\left\langle F_{z}\right\rangle$ is increased and moves downwards (smaller $p$ ) as $\left\langle F_{z}\right\rangle$ is decreased. Thus, by adjusting the condensate density, the magnetic bias field, and the total spin of the cloud, all regions of the spin-domain diagrams are accessible.

\subsection{Experimental methods for the study of spinor condensates}

Having introduced this new quantum fluid, let us describe how we made it, and how we probed it. First, magnetically-trapped Bose-Einstein condensates were produced in the $\left|F=1, m_{F}=-1\right\rangle$ hyperfine state and transferred to an optical trap [44. Then, we pulsed on rf fields of variable strength which were swept in frequency to distribute the optically-trapped atoms among the $F=1$ hyperfine sublevels by the method of adiabatic rapid passage [106]. High amplitudes or slow sweep rates transferred all the atoms from one hyperfine state to another, while low amplitudes or fast sweep rates transferred just a fraction of the atoms. To achieve an arbitrary hyperfine distribution, it was necessary to make these rf-transitions at large $(15-30 \mathrm{G})$ bias fields, separating the $\left|m_{F}=+1\right\rangle \rightarrow\left|m_{F}=0\right\rangle$ and $\left|m_{F}=0\right\rangle \rightarrow\left|m_{F}=-1\right\rangle$ transition frequencies by about $1 \mathrm{MHz}$ due to the quadratic Zeeman shift. Otherwise, at low fields where level spacings between the hyperfine sublevels are equal, rf fields can only be used to rotate the atomic spin vector and cannot, for example, change the atoms from the ferromagnetic $\left|m_{F}=+1\right\rangle$ state to the polar $\left|m_{F}=0\right\rangle$ state.

After state preparation, the optically-trapped spinor condensates were allowed to evolve in the presence of variable magnetic bias fields and field gradients. After a variable dwell time in the optical trap, the spinor condensates were probed by time-of-flight imaging combined with a Stern-Gerlach spin separation (Fig. 19). The optical trap was suddenly switched off, allowing the atoms to expand primarily radially from the highly anisotropic optical trap. Then, after allowing about $5 \mathrm{~ms}$ for the interaction energy to be completely converted to kinetic energy, a magnetic field gradient was applied which separated the spin state populations without distorting them. Finally, after $15-30$ $\mathrm{ms}$, the atoms were optically pumped to the $|F=2\rangle$ hyperfine manifold. This gave the same optical cross-section for all the atoms in the subsequent absorption probing on the $\left|F=2, m_{F}=2\right\rangle \rightarrow$ $\left|F^{\prime}=3, m_{F^{\prime}}=3\right\rangle$ cycling transition. This probing method obtained both the spatial and hyperfine distributions along the axis of the optical trap in a single image.

\subsection{The formation of ground-state spin domains}

In Ref. [97 we explored the ground-state structure of spinor Bose-Einstein condensates with an average spin of $\left\langle F_{z}\right\rangle=0$. Condensates were prepared either with all atoms in the $\left|m_{\mathrm{F}}=0\right\rangle$ state or in an equal mixture of the $\left|m_{\mathrm{F}}= \pm 1\right\rangle$ states. The atoms were then allowed to relax to their equilibrium distribution in the presence of a variable magnetic bias field and field gradient. Probing at variable 


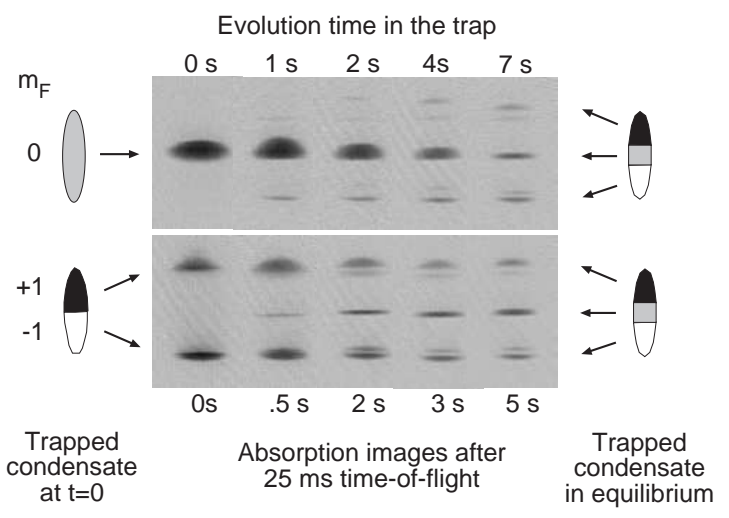

Fig. 20. Formation of ground state spin domains. Absorption images of ballistically expanding spinor condensates show both the spatial and hyperfine distributions. The images of clouds with various dwell times in the trap show the evolution to the same equilibrium for condensates prepared in either a pure $\left|m_{F}=0\right\rangle$ state (upper row) or in equally populated $\left|m_{F}= \pm 1\right\rangle$ states (lower row). The bias field and field gradient during the dwell time was $B_{0}=20 \mathrm{mG}$ and $B^{\prime}=11 \mathrm{mG} / \mathrm{cm}$. The image size for each spinor condensate is 1.7 $\times 2.7 \mathrm{~mm}$.

times after the state preparation revealed that the condensates relaxed to the same spin structure from either of the initial conditions, and remained thereafter in the same equilibrium state (Fig. 20).

Figure 21 shows three examples of the spin structures which were observed. The corresponding representations of these structures in the anti-ferromagnetic spin-domain diagram are indicated in Fig. 22 .

Fig. 21b shows the equilibrium structure of a spinor condensate for which the quadratic Zeeman energy is larger than the interaction energy $(q>c)$. The magnetic field gradient is sufficiently strong so that $|p|>q+c$ at the ends of the cloud, and thus the condensate consists of three pure spin domains with a $m_{F}=+1$ domain on the high-field end of the cloud, a $m_{F}=0$ domain in the center, and a $m_{F}=-1$ domain at the low-field end.

In Fig. 21 a, the magnetic field is weaker, and thus the central $m_{F}=0$ domain is now flanked by regions in which the $m_{F}= \pm 1$ components are mixed. The appearance of a large population of the $m_{F}=1\left(m_{F}=-1\right)$ component on the low-field (high-field) end of the cloud provides a qualitative confirmation of the anti-ferromagnetic collisional coupling in the $F=1$ hyperfine manifold of sodium. The division between the $m_{F}=0$ domain and the domains containing the $m_{F}= \pm 1$ components indicates the immiscibility of the $m_{F}=0$ component with the others.

Finally, in Fig. 21 c, a condensate is shown for which the total spin is greater than zero. The magnetic field gradient is about zero, and thus the condensate corresponds to a point on the spindomain diagram in which the $m_{F}= \pm 1$ components are mixed. This situation nicely demonstrates the miscibility of the $m_{F}= \pm 1$ components with each other. The different widths of the two components may be due to the curvature of stray magnetic fields, which has opposite effects on the $\left|m_{\mathrm{F}}= \pm 1\right\rangle$ states due to their different magnetic moments. Another explanation was recently given by Huang and Gou [107] who ascribe the different widths to the inhomogeneous density of the condensate.

There were some apparent discrepancies between our observations and the local density description (Sec. 4.3). For instance, as shown in Figs. 21 a and b, the separation between the central $m_{F}=0$ domain and the neighboring $m_{F}= \pm 1$ domains was not sharp, as would have been predicted by the spin-domain diagram which neglects the kinetic energy. Including the kinetic energy requires that the the condensate spinor vary gradually between the domains. As discussed in Secs. 4.7 and 4.8, the condensate spinor should change over a length scale given by $\xi_{s}=\sqrt{\hbar^{2} / 2 m \Delta E}$, where

$$
\Delta E=\mu_{0}\left(\sqrt{\frac{g_{0}+g_{2}}{g_{0}}}-1\right) \simeq 0.018 \mu_{0}
$$


(a)

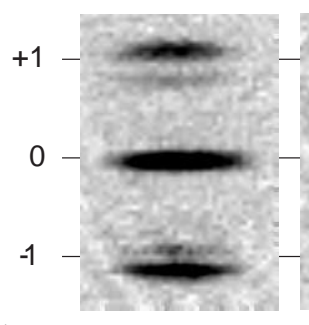

(b)
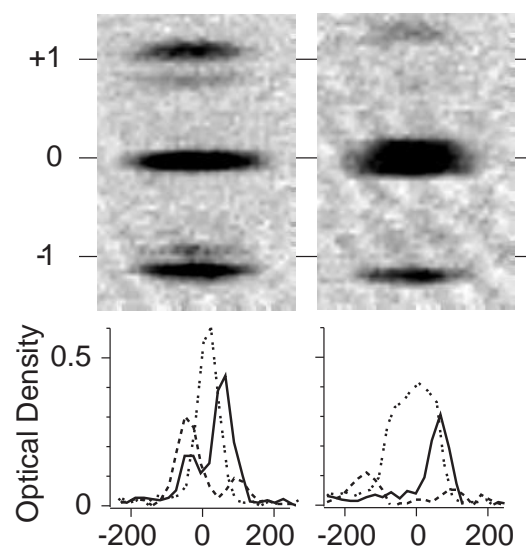

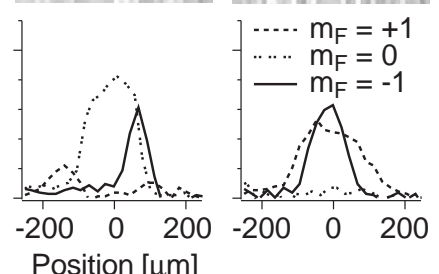

(c)

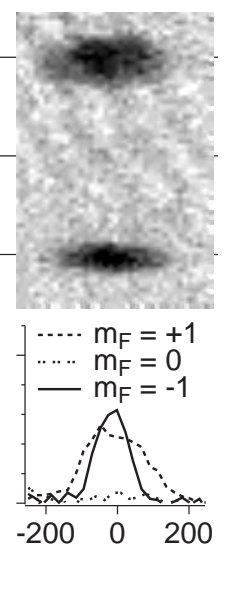

Fig. 21. Ground state spin domains in $F=1$ spinor Bose-Einstein condensates. Time-of-flight images after Stern-Gerlach separation are shown, along with the indicated axial density profiles in the optical trap, for which the Stern-Gerlach separation was "undone." Images (a) and (b) show spin domains of all three components. Image (c) shows a miscible $m_{F}= \pm 1$ component condensate. Conditions are: (a) $B=20 \mathrm{mG}$, $B^{\prime}=11 \mathrm{mG} / \mathrm{cm}$; (b) $B=100 \mathrm{mG}, B^{\prime}=11 \mathrm{mG} / \mathrm{cm} ;\left(\right.$ c) $B=20 \mathrm{mG}, B^{\prime} \simeq 0,\left\langle F_{z}\right\rangle>0$.

(a)

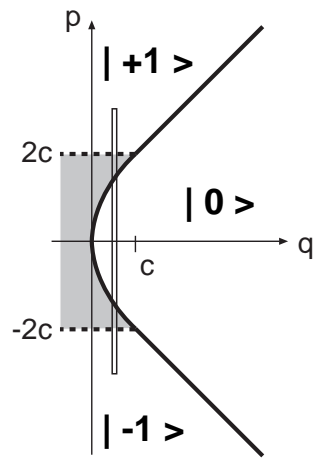

(b)

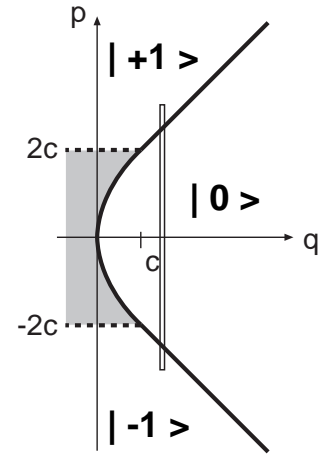

(c)

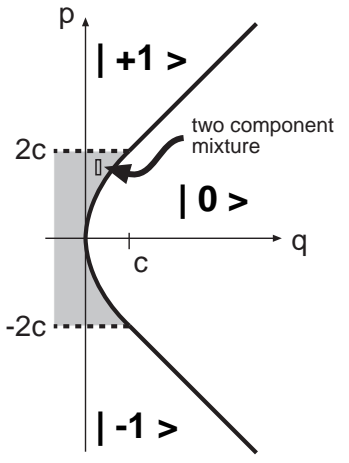

Fig. 22. Representation of ground-state spin-domain structures of Fig. 21 on the spin-domain diagram. The spin structures shown in Fig. 21 a and b correspond to long vertical lines through the spin-domain diagram, centered at $p=0$ to correspond to an average spin $\left\langle F_{z}\right\rangle=0$. (a) At low magnetic field $q<c$ and mixed-spin domains are introduced. (b) At higher fields, they are absent. (c) Lowering the gradient focuses on a small portion of the diagram in which a cloud of non-zero spin consists of overlapping $m_{F}= \pm 1$ components. 
is the height of the interaction energy barrier which expels $m_{F}=0$ atoms from $m_{F}=1$ spin domains, and $\mu_{0}=g_{0} n$ is the chemical potential of the $m_{F}=0$ atoms at a condensate density of $n$. This width $\xi_{s}$ can be called a "spin healing length" in analogy with the healing length $\xi=\sqrt{\hbar^{2} / 2 m \mu}$ which is the minimum length for density variations. For typical conditions, $\xi_{s} \simeq 1-2 \mu \mathrm{m}$.

In our time-of-flight images (Fig. 21), the overlap between the components appears to be much larger, on the order of tens of microns. This discrepancy may just be an artifact of the indirect probing technique from which the spin structure of the trapped condensate is inferred. During the expansion of the condensate, the kinetic energy at the spin-domain boundaries is released axially, imparting velocities of $\sqrt{2 \Delta E / m} \simeq 2 \mathrm{~mm} / \mathrm{s}$. During the $25 \mathrm{~ms}$ time of flight, this would cause a sharp boundary between spin components to be smeared out by $\simeq 50 \mu \mathrm{m}$, consistent with the observed width of the overlap between the $m_{F}=0$ and $m_{F}= \pm 1$ components. Thus, our time-offlight imaging technique cannot properly characterize the boundary between neighboring spin domains. In future work, it would be interesting to examine such boundaries with an in situ imaging technique, perhaps to observe the spatial structures recently predicted by Isoshima, Machida and Ohmi 108]. It is interesting to note that the radial expansion of the cigar-shaped condensate occurs at a velocity near the speed of Bogoliubov sound, which describes the propagation of density waves, while the axial expansion of a spin domain boundary occurs at a "spin sound velocity" which would describe the propagation of spin waves.

\subsection{Miscibility and immiscibility of spinor condensate components}

As discussed above, the spin-domain diagram and the observed ground-state spin structures showed evidence for the miscibility of the $m_{F}=-1$ and $m_{F}=+1$ components and the immiscibility of $m_{F}=$ \pm 1 and $m_{F}=0$ components. The bulk miscibility or immiscibility of two-component condensate mixtures is predicted by mean-field theory [75, 77 79, 109]. The interaction energy density of such condensates is given by

$$
E=\frac{1}{2}\left(n_{a}^{2} g_{a}+n_{b}^{2} g_{b}+2 n_{a} n_{b} g_{a b}\right)
$$

where $m$ is the common atomic mass, and $n_{a}$ and $n_{b}$ are the densities of each of the components. The interaction parameters are given generally as $g=4 \pi \hbar^{2} a / m$ where $a_{a}$ and $a_{b}$ are the samespecies scattering lengths, and $a_{a b}$ is the scattering length for interspecies collisions. Consider a two-component mixture in a box of volume $V$ with $N$ atoms in each component. If the condensates overlap, their total mean-field energy is

$$
E_{O}=\frac{N^{2}}{2 V}\left(g_{a}+g_{b}+2 g_{a b}\right)
$$

If they phase separate, their energy is

$$
E_{S}=\frac{N^{2}}{2}\left(\frac{g_{a}}{V_{a}}+\frac{g_{b}}{V_{b}}\right)
$$

The volumes $V_{a}$ and $V_{b}$ occupied by each of the separated condensates are determined by the condition of equal pressure:

$$
g_{a}\left(\frac{N}{V_{a}}\right)^{2}=g_{b}\left(\frac{N}{V_{b}}\right)^{2}
$$

Comparing the energies $E_{O}$ and $E_{S}$ the condensates will phase-separate if $g_{a b}>\sqrt{g_{a} g_{b}}$, and will mix if $g_{a b}<\sqrt{g_{a} g_{b}}$.

In the $F=1$ three-component spinor system, the scattering lengths are determined by $a_{f=0}$ and $a_{f=2}$. Defining $\bar{a}=\left(2 a_{f=2}+a_{f=0}\right) / 3$ and $\Delta a=\left(a_{f=2}-a_{f=0}\right) / 3$, the scattering lengths for the $m_{F}=1,0$ two-component system (or equivalently the $m_{F}=-1,0$ system) are given by $a_{0}=\bar{a}$, and $a_{1}=a_{0,1}=\bar{a}+\Delta a$. Since $\Delta a$ is positive for sodium, the condition $a_{0,1}>\sqrt{a_{0} a_{1}}$ applies and the components should phase-separate, as we have observed [97, 98]. Interestingly, this phase-separation should not occur in the non-condensed cloud because the same-species mean-field interaction energies are doubled due to exchange terms. 
In the $m_{F}= \pm 1$ two component system, the scattering lengths are $a_{1}=a_{-1}=\bar{a}+\Delta a$ and $a_{1,-1}=\bar{a}-\Delta a$. Thus, $a_{1,-1}<\sqrt{a_{1} a_{-1}}$, and these two components should mix. Indeed, as shown in Fig. 21c, an equilibrium spinor condensate with $\left\langle F_{z}\right\rangle \neq 0$, small field gradient, and near-zero field consists of an overlapping mixture of atoms in the $m_{F}= \pm 1$ states. This particular miscible two-component system has an important advantage. If the trapping potential varies across a twocomponent condensate, the lowest energy state may be a phase-separated state if $a_{a} \neq a_{b}$ even though the condition $a_{a b}<\sqrt{a_{a} a_{b}}$ is fulfilled [110]. In this case, the atoms with the smaller scattering length concentrate near the trap center, making it harder to observe miscibility. However, in the $m_{F}= \pm 1$ system, the two scattering lengths $a_{1}$ and $a_{-1}$ are equal by rotational symmetry, so the components mix completely even in a trapping potential.

\subsection{Metastable states of spinor Bose-Einstein condensates}

Having observed and explained ground state spin-domain structures, we began to explore dynamical properties of spinor Bose-Einstein condensates. Ref. 98 discusses the observation of long-lived excited states of spinor Bose-Einstein condensates. We observed two complementary types of metastable states: one type in which a two-component condensate was stable in spin composition but which persisted in a non-equilibrium structure of spin domains, and another in which a spatially uniform condensate was metastable with respect to spin relaxation to an equilibrium spin composition. In each case, the energy barriers to relaxation to the ground state (the activation energy) were identified and found to be much smaller than the thermal energies of the metastable gases; thus, such states would not be metastable in a non-condensed cloud. However, in a Bose condensed cloud with a large condensate fraction, the thermal energy is only available to the scarce thermal component and thus thermal relaxation is considerably slowed. In other words, Bose-Einstein condensation allows the study of weak effects in an energy regime which is much lower than the temperature of the gas.

\subsubsection{Metastable spin-domain structures}

The first type of metastability was observed in spinor Bose-Einstein condensates in a high magnetic field $(15 \mathrm{G})$ which caused a two-component $m_{F}=0,1$ cloud to be stable in spin composition. This occurs because the large quadratic Zeeman shift makes $\left|m_{\mathrm{F}}=0\right\rangle+\left|m_{\mathrm{F}}=0\right\rangle \rightarrow\left|m_{\mathrm{F}}=+1\right\rangle+\left|m_{\mathrm{F}}=-1\right\rangle$ collisions endothermic, even if the $\left|m_{\mathrm{F}}= \pm 1\right\rangle$ atoms would move to the ends of the condensate. We prepared clouds in an equal superposition of the two hyperfine states using a brief rf pulse, and then allowed the system to equilibrate.

Because of the immiscibility of the $m_{F}=0$ and $m_{F}=1$ components, the ground-state spin structure in this case consists of two phase-separated spin domains, one for each of the components, on opposite sides of the elongated trap with a domain boundary in the middle. What we observed was dramatically different: a spontaneously formed, metastable arrangement of alternating $m_{F}=0$ and $m_{F}=1$ spin domains (Fig. 23).

These spin striations began forming within about $50 \mathrm{~ms}$ of the initial preparation of two overlapping, immiscible components. The striations were initially angled due to radial excitations in the narrow spinor condensates which soon damped out, leaving strictly horizontal striations. The observed width of the spin domains grew to an equilibrium value of about $40 \mu \mathrm{m}$ within about $100 \mathrm{~ms}$ (Fig. 24.). Thereafter, the clouds were essentially unchanged, remaining in the metastable state for 10 or more seconds as the number of trapped atoms slowly decayed due to three-body trap losses.

To understand the reason for the metastability, let us consider two adjacent spin domains of the $m_{F}=0$ and $m_{F}=1$ components, as shown in Fig. 25. Suppose that, in order for the condensate to decay to its ground state, the atoms of each spin domain must somehow be transported across the spin-domain boundary. For this to occur, the atoms of one component must either pass around or through the other component.

If one component passes around the other, the condensate wavefunction must be varied spatially in the radial direction. This gives a kinetic energy barrier to decay of about $\hbar^{2} / 2 m r^{2} \simeq k_{B} \times 3$ nK where $r \simeq 2 \mu \mathrm{m}$ is the condensate radius. The passage of one component through the other is limited by an interaction barrier since the components are immiscible. As explained in Ref. [99], the 

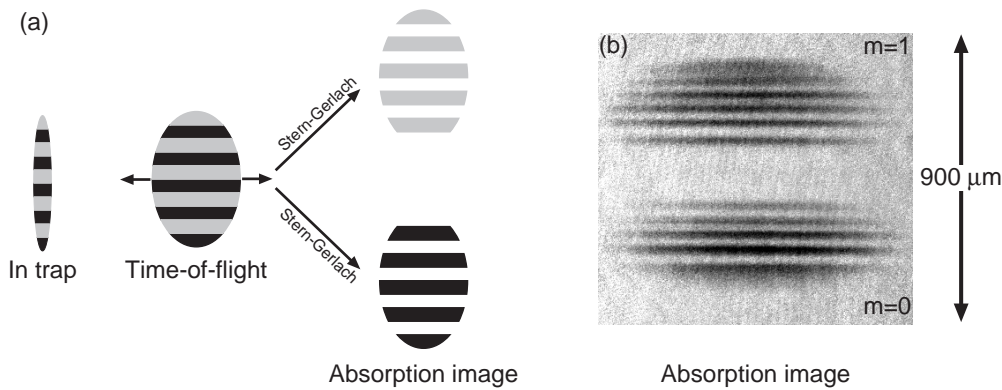

Fig. 23. Quantum "bubbles": a metastable arrangement of alternating spin domains in a two-component condensate. (a) A two-component optically-trapped condensate composed of atoms in the $\left|m_{\mathrm{F}}=0\right\rangle$ (black) and $\left|m_{\mathrm{F}}=1\right\rangle$ (grey) hyperfine states expands primarily radially once the trap is switched off. A magnetic field gradient is used to separate the two spin states before imaging, allowing a determination of the axial distribution of the two components in the trap. (b) The observed density striations indicate that the trapped condensate was composed of alternating $m_{F}=0$ and $m_{F}=1$ spin domains.

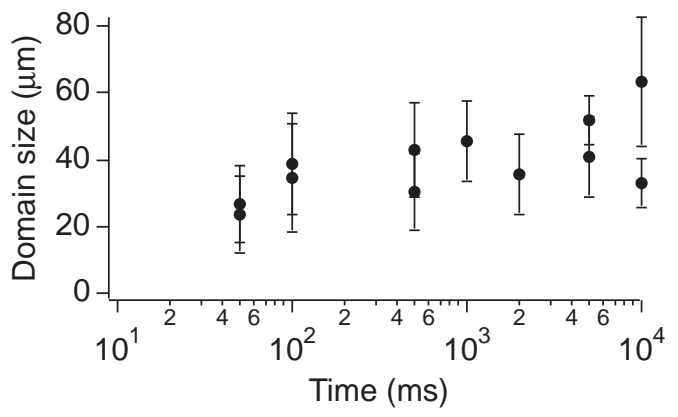

Fig. 24. Evolution of metastable domain sizes. Shown is the average size (points) and standard deviation (one sigma error bars) of the spin domains observed at various time after the initial state preparation at a bias field of $15 \mathrm{G}$. Each point corresponds to measurements made on a different condensate. 


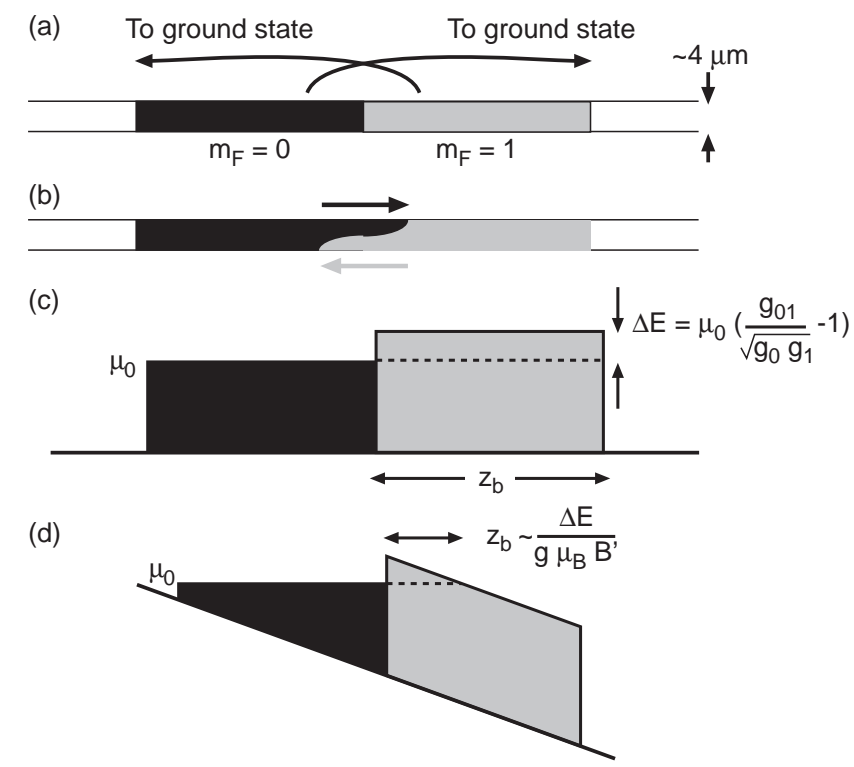

Fig. 25. Energy barriers responsible for the metastability of spin domains in a two-component $m_{F}=0,1$ condensate. (a) Metastable domains of the $m_{F}=0$ (black) and $m_{F}=1$ (gray) components are held in a narrow optical trap. For the population in these domains to decay to the ground state they must either pass each other without overlapping, or pass through each other. (b) The former is prohibited due to the kinetic energy barrier of modifying the condensate wavefunction on a radial length scale. (c) The latter is prohibited due to an interaction energy barrier $\Delta E$, shown here for the passage of an $m_{F}=0$ domain of chemical potential $\mu_{0}$ through the neighboring $m_{F}=1$ domain. (d) If a magnetic field gradient $B^{\prime}$ is imposed, the width of the classically forbidden region for the passage of $m_{F}=0$ atoms through the $m_{F}=1$ domain is reduced to $z_{b}=\Delta E / g \mu_{B} B^{\prime}$, and the tunneling rate which governs the decay of the metastable domains is increased. 
interaction energy barrier for atoms in the $\left|m_{\mathrm{F}}=0\right\rangle$ state to pass through a $m_{F}=1$ domain is (see Eq. 4.19) $\Delta E \simeq g_{2} / 2 g_{0} \times \mu_{0}=c$ where $\mu_{0}$ is the chemical potential of atoms in the $m_{F}=0$ spin domain, and $c$ is the spin-dependent interaction energy introduced in Sec. 4.1. At a chemical potential $\mu_{0}=k_{B} \times 300 \mathrm{nK}$, the interaction energy barrier height is $c \simeq k_{B} \times 5 \mathrm{nK}$. The probability of tunneling through the spin domains is exceedingly small because the axial length of the domains $z_{B} \simeq 40 \mu \mathrm{m}$ is much larger than the spin healing length $\xi_{s} \simeq 1.4 \mu \mathrm{m}$. Thus, because of these two energy barriers, the non-equilibrium arrangement of spin-domains is metastable.

The formation of these metastable spin-domains was considered recently by Chui and Ao [111] as a spinodal decomposition in a binary system. Equilibration occurs on two time scales. First, on a short time scale determined by the spin-dependent interaction energy $c$ as $t \simeq \hbar / c$, the homogeneous initial state begins to phase separate into small domains of length scale $\xi_{s}=\sqrt{\hbar^{2} / 2 m \Delta E}$. Thereafter, the domains grow by the coalescence of small spin domains on the long time scales required for quantum tunneling. This work supports the physical picture which we suggested in Ref. 988. A similar picture emerges from the work of $\mathrm{Pu}$ et al. [112] who consider the quantum dynamics (without dissipation) of a trapped condensate composed of two overlapping components which tend to phase separate. In their calculations, they observe the evolution of fine spatial features which exemplify the instability of such a system against spin domain formation.

\subsubsection{Metastable spin composition}

Another type of metastability was discovered in the studies of ground-state spin domains of a $\left\langle F_{z}\right\rangle=0$ condensate discussed in Sec. 4.5. Condensates were prepared either with all atoms in the $\left|m_{\mathrm{F}}=0\right\rangle$ state or in an equal mixture of the $\left|m_{\mathrm{F}}= \pm 1\right\rangle$ states. While the ground state reached from either starting condition was the same (Fig. 20), equilibration occurred on much different time scales. When starting from the $\left|m_{F}=0\right\rangle$ state, the condensate remained unchanged for several seconds before evolving over the next few seconds to the ground state. When starting from the $\left|m_{F}= \pm 1\right\rangle$ superposition, the fraction of atoms in the $\left|m_{F}=0\right\rangle$ state grew without delay, arriving at equilibrium within less than a second (Fig. 26).

This difference can be understood by considering a spin-relaxation collision, in which two $\left|m_{\mathrm{F}}=0\right\rangle$ atoms collide to produce a $\left|m_{\mathrm{F}}=1\right\rangle$ and a $\left|m_{\mathrm{F}}=-1\right\rangle$ atom. In the presence of a magnetic field $B_{0}$, quadratic Zeeman shifts cause the energy of the two $\left|m_{\mathrm{F}}=0\right\rangle$ atoms to be lower than that of the $\left|m_{\mathrm{F}}=1\right\rangle$ and $\left|m_{\mathrm{F}}=-1\right\rangle$ atoms. Due to this activation energy, condensate atoms in the $\left|m_{\mathrm{F}}=0\right\rangle$ state could not undergo spin-relaxation collisions. Thus, even though the creation of $m_{F}=1$ and $m_{F}=-1$ spin domains at the ends of the condensate is energetically favored globally in the presence of a magnetic field gradient, the $m_{F}=0$ condensate cannot overcome the local energy barrier for spin-relaxation. In contrast, condensate atoms in the $\left|m_{\mathrm{F}}=1\right\rangle$ and $\left|m_{\mathrm{F}}=-1\right\rangle$ states can directly lower their energy through such collisions, and equilibrate quickly.

In support of this explanation, the metastability time was found to depend strongly on the quadratic Zeeman energy which was varied by changing the magnetic bias field. Importantly, the equilibration time changed significantly when $q$ was varied by less than a nanokelvin; this dependence excludes thermal spin relaxation (in a gas at a temperature $T \sim 100 \mathrm{nK} \gg q / k_{B}$ ) as the equilibration mechanism, and suggests that the metastable condensate decays to the ground state via quantum tunneling. In such tunneling, a pair of atoms in the $\left|m_{\mathrm{F}}= \pm 1\right\rangle$ states would be produced in a classically forbidden collision, and would then tunnel to opposite ends of the cloud where their energy is lowered due to the magnetic field gradient. It would be interesting to study this process further in the future.

\subsection{Quantum tunneling}

Metastable states can generally overcome the activation energy barrier for decay to the ground state in two ways. Classically, the system can decay by acquiring thermal energy larger than the activation energy. However, even without this thermal energy, the system can decay to the ground state by quantum tunneling. Tunneling describes a wide range of phenomena such as nuclear decay, field ionization of neutral atoms, and scanning tunneling microscopy. In macroscopic quantum systems, 


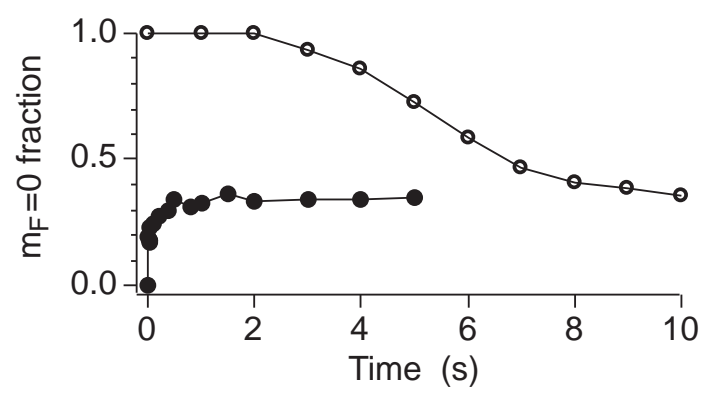

Fig. 26. Metastability of the pure $\left|m_{F}=0\right\rangle$ state in the presence of a magnetic bias field (250 mG), and gradient $(44 \mathrm{mG} / \mathrm{cm})$. The evolution toward equilibrium of an initially pure $\left|m_{F}=0\right\rangle$ condensate (open symbols), and a mixture of $\left|m_{F}=1\right\rangle$ and $\left|m_{F}=-1\right\rangle$ (closed symbols) is shown by plotting the fraction of atoms in the $\left|m_{F}=0\right\rangle$ state vs. dwell time in the optical trap. Figure taken from Ref. 98.

coherent tunneling can lead to a variety of Josephson effects [113] which have been observed in superconductors and quantum fluids. As exemplified by the observation of metastable states which persist in spite of temperatures higher than the activation energy, gaseous Bose-Einstein condensates are an appealing new system to study tunneling and Josephson oscillations 114 117.

In our study of optically trapped spinor Bose-Einstein condensates, we looked at the decay of metastable spin domains via quantum tunneling through the spin domain boundaries [99]. Tunneling barriers were formed not by an external potential, but rather by the intrinsic repulsion between two immiscible components of a quantum fluid. Tunneling across spin domain boundaries is a spin transport mechanism inherent to such a fluid (emphasized in [111]), and the tunneling rates are sensitive probes of the structure of the domain boundaries. From a practical viewpoint, the use of phase-separated spin domains rather than externally imposed potentials is an attractive option for future studies of tunneling in Bose condensates since the energy barriers for tunneling are naturally of nanokelvin-scale height and micron-scale width.

The system we chose for our study was a simple, well-characterized metastable arrangement of spin domains in a spinor condensate composed of the $m_{F}=1$ and $m_{F}=0$ components. Such a state was obtained by first preparing a spinor condensate in a superposition of the $\left|m_{\mathrm{F}}=0,1\right\rangle$ states. A strong field gradient (several $\mathrm{G} / \mathrm{cm}$ ) was applied to break up the many-domain metastable state (discussed in the previous section) and separate the spin components into the two-domain ground state. Then, a weak gradient was applied in the opposite direction, which energetically favored the rearrangement of the spin domains on opposite ends of the optical trap, and thus yielded a two-domain metastable state. This simple system allowed for the characterization of the decay of metastable states by the easy identification of atoms in the metastable and ground-state spin domains of each component (Fig. 27).

To ascribe the decay of these metastable spin domains to quantum tunneling, it was first necessary to rule out thermal relaxation as a decay mechanism. Figure 28 shows a series of time-of-flight images reflecting the state of the two-domain metastable state at various times after the state was initially prepared and held under a $0.1 \mathrm{G} / \mathrm{cm}$ gradient. One can identify two stages in the decay of this metastable cloud to the ground state: a slow decay over the first 12 seconds, followed by a rapid decay to the ground state within less than one second. The slow decay was found to be rather insensitive to changes in the condensate density and field gradient, and was thus consistent with thermal relaxation wherein condensate atoms from the metastable state are thermally excited, and then re-condense into the ground state domains. The number of atoms which accumulated in the ground-state domains reached a nearly constant value of about $5 \times 10^{4}$, perhaps due to a dynamic equilibrium between the growth of the domain via re-condensation and its depletion via inelastic losses. We also observed that the total population in the $\left|m_{\mathrm{F}}=0\right\rangle$ spin state decreases more rapidly than that in the $\left|m_{\mathrm{F}}=1\right\rangle$ state, indicating higher inelastic collision rates for the $\left|m_{\mathrm{F}}=0\right\rangle$ state. 


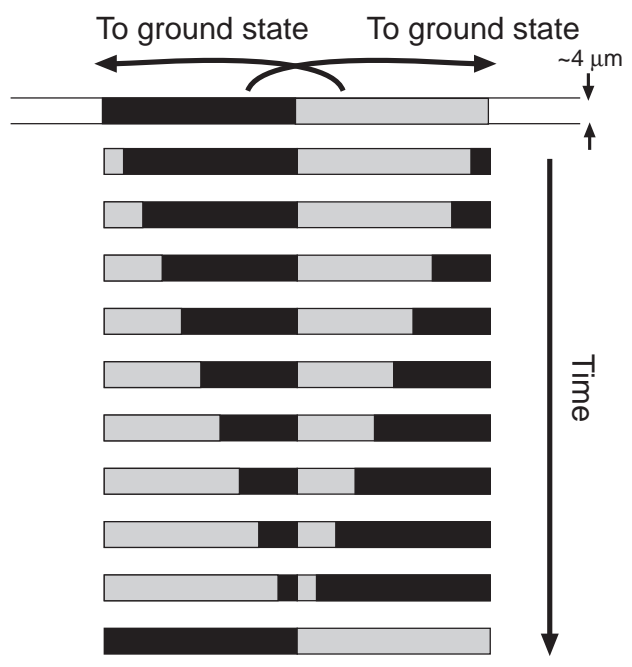

Fig. 27. Evolution from a metastable state to the ground state. The ground state domain structure is formed by tunneling of the two components through each other. The intermediate state is characterized by outer ground state domains and inner metastable domains.

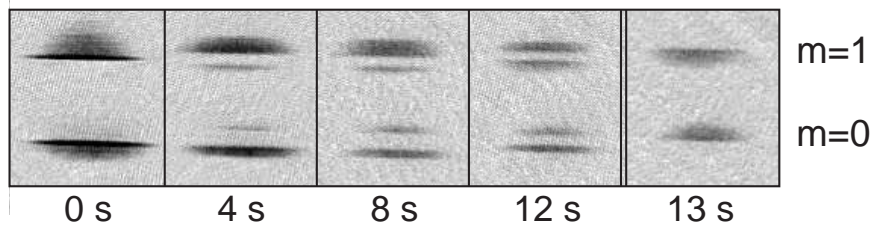

Fig. 28. Decay of a metastable state by thermal relaxation and quantum tunneling. A two-domain metastable state was prepared at a $15 \mathrm{G}$ axial bias field and a $0.1 \mathrm{G} / \mathrm{cm}$ gradient. Images show metastable (outer parts in the time-of-flight picture) and ground state (inner parts) spin domains of $\left|m_{F}=1\right\rangle$ and $\left|m_{F}=0\right\rangle$ atoms, probed at various times after state preparation. The height of each image is $1.3 \mathrm{~mm}$. The number of atoms in the ground state domains grew slowly over the first $12 \mathrm{~s}$, after which the atoms tunneled quickly to the ground state. The total number of atoms (and thus the condensate density) decreased during the dwell time due to inelastic three-body collisions. Thus, the slow thermal relaxation gave way to a rapid tunneling once the density fell below a threshold value. Figure taken from Ref. [98]. 


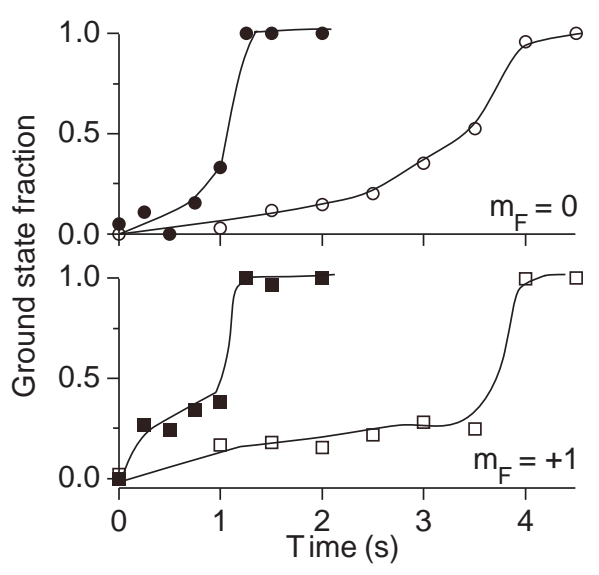

Fig. 29. A comparison of the decay of metastable states at different densities. Shown is the fraction of $m_{F}=0$ and $m_{F}=1$ atoms found in the ground-state domains after a variable delay time at a constant field gradient of $0.35 \mathrm{G} / \mathrm{cm}$ and a $2 \mathrm{G}$ bias field. The evolution is shown for condensates prepared with different populations in the initial state, either $1 \times 10^{6}$ (open symbols) or $2.5 \times 10^{5}$ atoms (closed symbols). The rapid tunneling in the denser condensate occurred at a later time. The solid lines are guides to the eye.

The rapid decay which ensued (after 12 seconds) was due to quantum tunneling. As discussed below, the tunneling rate is acutely sensitive to the condensate density. While the condensate is stored in the optical trap, its density decreases as atoms are lost from the trap due to three-body inelastic collisions. Thus, as time progressed, the density of the metastable condensate in Fig. 28 decreased until the tunneling rate was fast enough to cause a rapid (within about 1 second) relaxation to the ground state.

The dependence of the relaxation time on the condensate density is also shown in Fig. 29. Metastable condensates were prepared with different initial numbers of atoms, and held in a constant magnetic field gradient. The metastable lifetimes for the two starting conditions were different, with the denser condensate decaying to the ground state at a later time.

A mean-field description of the tunneling rates was developed [99]. We considered the onedimensional motion of a Bose-Einstein condensate composed of two immiscible components of atoms in states $|a\rangle$ and $|b\rangle$ and atomic mass $m$, as in Fig. 25 for the experimental realization of the $m_{F}=1$ and $m_{F}=0$ components. The chemical potentials of the two components $\mu_{i}=g_{i} n_{i}$ are related by the condition of constant pressure $\mu_{a}^{2} / g_{a}=\mu_{b}^{2} / g_{b}$ where $g_{i}=4 \pi \hbar^{2} a_{i} / m, a_{i}$ is the same-species scattering length, and $i \in a, b$ labels the component. The mean-field interaction energy for each component is $U_{i}=g_{i} n_{i}+g_{a b} n_{j}(i \neq j)$ where $g_{a b}$ is determined by the cross-species scattering length $a_{a b}$.

If we assume the boundary between the spin domains to be sharp, component $a$ is excluded from the domain of component $b$ by an energy barrier $\Delta E$ given by

$$
\Delta E=g_{a b} n_{b}-g_{a} n_{a}=\left(\frac{g_{a b}}{\sqrt{g_{a} g_{b}}}-1\right) \mu_{a}
$$

This energy barrier is responsible for the metastability of spin domains which have an axial length $z_{b}$ which is much larger than the spin healing length $\xi_{s}=\sqrt{\hbar^{2} / 2 m \Delta E}$.

In the presence of a state-selective force $F$, which was experimentally applied in our experiments using a magnetic field gradient $B^{\prime}$, the energy barrier decreases away from the domain boundary as $\Delta E(z)=\Delta E-F z$. Thus the width of the energy barrier is reduced to $z_{b}=\Delta E / F$, and tunneling can occur when $z_{b} \sim \xi_{s}$. More precisely, the tunneling rate across the barrier is given by the FowlerNordheim (WKB) equation

$$
\frac{d N_{a}}{d t}=\gamma \exp \left(-2 \sqrt{\frac{2 m}{\hbar^{2}}} \int_{0}^{z_{b}} \sqrt{\Delta E(z)} d z\right)
$$




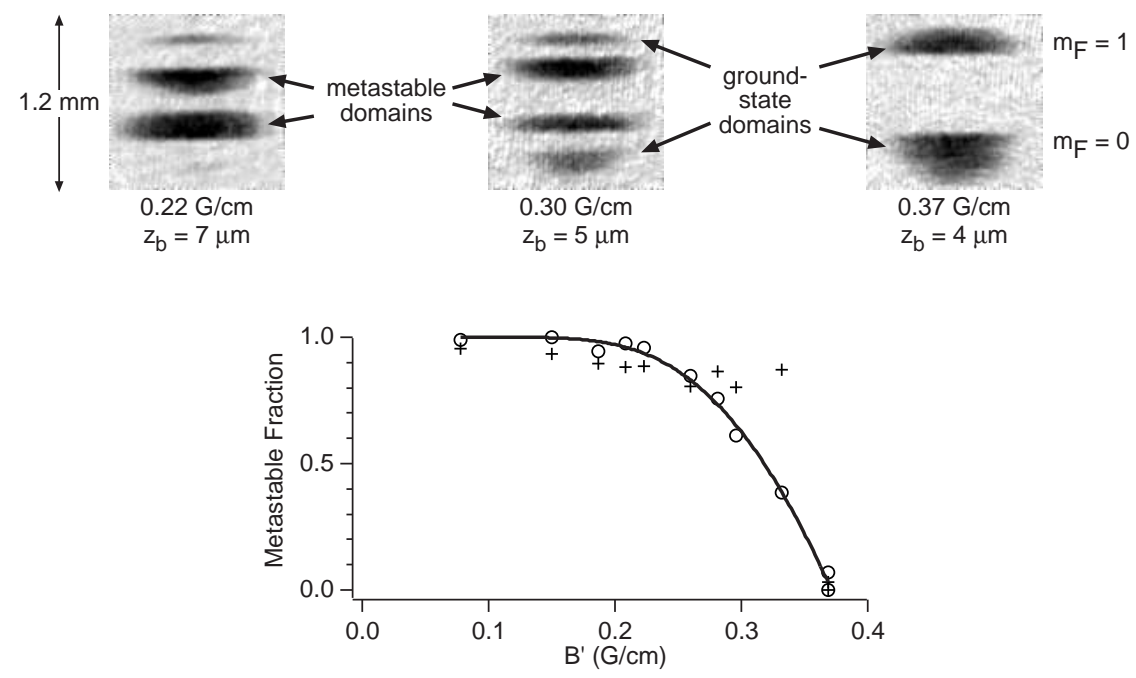

Fig. 30. Tunneling across barriers of constant height and variable width $z_{b}$. Condensates at constant density were probed after 2 seconds of tunneling at a variable field gradient $B^{\prime}$. The population of atoms in the metastable and ground-state domains of each spin state were easily identified in time-of-flight images (see Fig. 27). Also shown is the fraction of atoms of each spin state in their metastable domain. Circles represent the $m_{F}=0$ atoms, and pluses the $m_{F}=1$ atoms. The energy barrier height was $\approx 5 \mathrm{nK}$ at the chemical potential of $\mu_{0} \simeq 300 \mathrm{nK}$. The tunneling rate depended strongly on the width of the energy barrier $z_{b}=\Delta E / g \mu_{B} B^{\prime}:$ at $z_{b}=7 \mu \mathrm{m}\left(B^{\prime}=0.22 \mathrm{G} / \mathrm{cm}\right)$ little tunneling was observed, while at $z_{b}=4 \mu \mathrm{m}\left(B^{\prime}=0.37\right.$ $\mathrm{G} / \mathrm{cm}$ ) the atoms had completely tunneled to the ground state in $2 \mathrm{~s}$. The barrier attempt rate and tunneling probability were determined by a fit to the $m_{F}=0$ data (solid line), and found to agree with a mean-field model. The data indicate that the tunneling rate for $m_{F}=0$ atoms is larger than that for $m_{F}=1$ atoms.

$$
\begin{aligned}
& =\gamma \exp \left(-\frac{4}{3} \sqrt{\frac{2 m}{\hbar^{2}}} \frac{\Delta E^{3 / 2}}{F}\right) \\
& =\gamma \exp \left(-\frac{4}{3} \frac{z_{b}}{\xi_{s}}\right)
\end{aligned}
$$

which also describes the analogous phenomenon of the field emission of electrons from cold metals [118]. Here $\gamma$ is the total tunneling attempt rate (i.e. not the rate per particle), and the exponential is the tunneling probability. This relation explains the strong dependence of the tunneling rate on the condensate density $n$, which causes the density threshold behaviour observed in Fig. 28.

This mean-field description was tested experimentally by measuring the tunneling rate across barriers of constant height and variable width. For this, metastable condensates were prepared at a constant density (giving a constant barrier height of $\approx 5 \mathrm{nK}$ ), exposed to a variable gradient (giving barrier widths between 4 and $20 \mu \mathrm{m}$ ), and allowed to decay for a period $\tau=2 \mathrm{~s}$ which was short enough that the condensate density did not vary appreciably due to trap losses. We then measured the number of atoms of each component in the metastable and ground-state spin domains by time-offlight absorption imaging (Fig. 30). When the barrier was wide (small field gradients), the tunneling rate was small and only a small fraction of atoms were observed in the ground-state domains. When the barrier was narrow (large field gradients), the tunneling rate was large and a large fraction of atoms were observed in the ground-state domains. The data were in quantitative agreement with our mean-field approach using the scattering lengths calculated by Burke et al. [105]. 


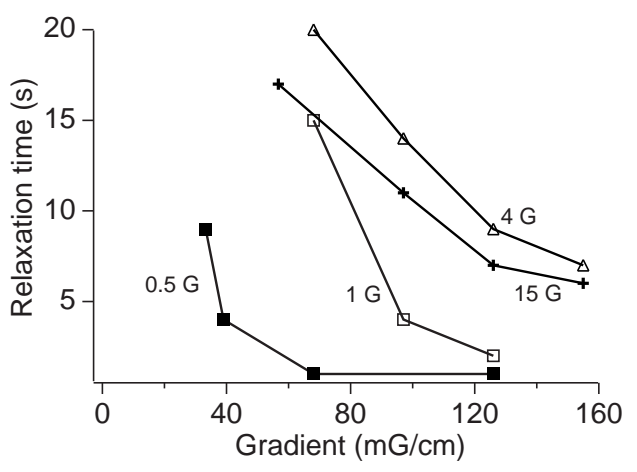

Fig. 31. Variation of the metastable state lifetime with magnetic field and field gradient. Metastable condensates at a constant initial chemical potential of $\mu_{0} \simeq 600 \mathrm{nK}$ were allowed to decay at a variable bias field and field gradient. Shown is the time at which the condensates were observed to have relaxed completely to the ground state. The dependence of the lifetime on the field gradient at $15 \mathrm{G}$ and $4 \mathrm{G}$ was similar. At fields of $1 \mathrm{G}$ or lower, the lifetime was dramatically shortened, indicating a substantially higher tunneling rate at low magnetic fields.

\subsection{Magnetic field dependence of spin-domain boundaries}

While the tunneling rates measured at high magnetic fields (15 G) agreed with a model of tunneling in a two-component Bose condensate, at lower magnetic fields, a dramatic increase in the tunneling rate was observed indicating the breakdown of the two-component description. As described in our paper (see Fig. 4 of Ref. 99]), the threshold chemical potential for tunneling at a constant field gradient dramatically increased at magnetic fields below about 1 G. Fig. 31 shows similar data. This strong increase in the tunneling rate at low fields reveals that the structure of the domain boundary is changed by the presence of the third spin component, the $\left|m_{\mathrm{F}}=-1\right\rangle$ state.

The introduction of the third spin component to the barrier has the effect of reducing the energy barrier for the tunneling of $m_{F}=0$ atoms. This can be seen by considering a mixture of components $a$ and $b$ where $|a\rangle=\left|m_{\mathrm{F}}=0\right\rangle$ and $|b\rangle=\cos \theta\left|m_{\mathrm{F}}=1\right\rangle-\sin \theta\left|m_{\mathrm{F}}=-1\right\rangle(0 \leq \theta \leq \pi / 2)$. The interaction energy density of this system can be written in the form of Eq. 4.20 with the definitions

$$
\begin{aligned}
g_{a} & =g_{0} \\
g_{b} & =g_{0}+\Delta g \cos ^{2} 2 \theta \\
g_{a b} & =g_{0}+\Delta g(1-\sin 2 \theta)
\end{aligned}
$$

where $\Delta g=g_{2}$. Using these interaction parameters, the energy barrier height which governs the tunneling of the $m_{F}=0$ component becomes

$$
\Delta E(\theta)=\left(\frac{g_{0}+\Delta g(1-\sin 2 \theta)}{\sqrt{g_{0}\left(g_{0}+\Delta g \cos ^{2} 2 \theta\right)}}-1\right) \mu_{0} \simeq \frac{\Delta g}{g_{0}}\left(1-\sin 2 \theta-\frac{\cos ^{2} 2 \theta}{2}\right) \mu_{0}
$$

for $\Delta g \ll g_{0}$. For $\theta=0$ or $\theta=\pi / 2$, one recovers the energy barrier for tunneling through a pure $m_{F}=1$ or $m_{F}=-1$ barrier. For intermediate values, the height of the energy barrier is reduced. Indeed, at $\theta=\pi / 4$, the energy barrier disappears completely. In this case, $|b\rangle=\frac{1}{\sqrt{2}}\left(\left|m_{\mathrm{F}}=1\right\rangle-\mid m_{\mathrm{F}}=\right.$ $-1\rangle)$ describes a polar state obtained by rotating the $\left|m_{\mathrm{F}}=0\right\rangle$ state. The condensate spinor can thus evolve continuously from state $|a\rangle$ to $|b\rangle$ by a gradual rotation which is associated with a gap-less (i.e. no barrier) Goldstone excitation mode. Recent calculations by Isoshima, Machida and Ohmi [108] confirm the disappearance of the energy barrier when the populations in the $\left|m_{\mathrm{F}}= \pm 1\right\rangle$ states become equal. These changes of effective interaction parameters in the presence of the $\left|m_{\mathrm{F}}=-1\right\rangle$ state provide a mechanism for the variation of the tunneling rates with magnetic fields. 
The local density approximation discussed in Sec. 4.3 indicates that, indeed, the $m_{F}=-1$ component should be introduced to the spin domain boundaries at low magnetic fields. According to this approximation, the boundaries studied in our experiment between an $m_{F}=0$ domain and a region of $\left\langle F_{z}\right\rangle>0$ are described in the spin domain diagram (Fig. 22) by a short vertical line at constant $q$ (determined by the magnetic field) which straddles the border of the $m_{F}=0$ region in the $p>0$ half-plane. The diagram thus suggests that a pure two-component description of the boundary is correct for magnetic fields at which the quadratic Zeeman energy is larger than the spin-dependent interaction energy $(q>c)$. Taking a typical value of the chemical potential of $\mu_{0}=300 \mathrm{nK}$, this condition indicates that the two-component description of tunneling should be valid for magnetic fields greater than $500 \mathrm{mG}$. Below this magnetic field, the $m_{F}=0$ component is bordered not by a pure $m_{F}=1$ domain, but rather by a region in which the $\left|m_{\mathrm{F}}=1\right\rangle$ and $\left|m_{\mathrm{F}}=-1\right\rangle$ states are mixed.

However, the local density approach fails to explain why the tunneling rates should increase even at magnetic fields for which $q>c$, as we observed. This failure stems from the incomplete description of the spin domain boundary due to the neglect of kinetic energy in the local density approximation. As discussed above, $\left\langle F_{z}\right\rangle$ must vary continuously at the boundary between spin domains. One may approximate the spin composition of the boundary region by explicitly minimizing the energy of a spinor with a given value of $\left\langle F_{z}\right\rangle$, and then applying a local density approximation in which we assume that the spinor at each location in the boundary is determined by the local value of $\left\langle F_{z}\right\rangle$. We can write a spinor for which $\left\langle F_{z}\right\rangle>0$ as

$$
\vec{\zeta}=\left(\begin{array}{c}
\sqrt{\left\langle F_{z}\right\rangle+\epsilon^{2}} \\
\sqrt{1-\left\langle F_{z}\right\rangle-2 \epsilon^{2}} \\
-\epsilon
\end{array}\right)
$$

where a choice of complex phases has already been made which reduces the anti-ferromagnetic interaction energy. The spin-dependent energy $K_{\text {spin }}$ is then

$$
c\left(\left\langle F_{z}\right\rangle^{2}+2\left(1-2 \epsilon^{2}-\left\langle F_{z}\right\rangle\right)\left(\sqrt{\epsilon^{2}+\left\langle F_{z}\right\rangle}-\epsilon\right)^{2}\right)+q\left(2 \epsilon^{2}+\left\langle F_{z}\right\rangle\right)
$$

which is minimized to determine the fractional population $\epsilon^{2}$ in the $\left|m_{\mathrm{F}}=-1\right\rangle$ state.

At high magnetic fields $(q \gg c)$ one finds the approximate solution

$$
\epsilon^{2} \simeq \frac{1}{(q / c)^{2}}\left\langle F_{z}\right\rangle\left(1-\left\langle F_{z}\right\rangle\right)^{2}
$$

which indicates that atoms in the $\left|m_{\mathrm{F}}=-1\right\rangle$ state are always energetically favored to reside in the domain boundary 5 . Their population scales with the magnetic field $B_{0}$ as $q^{-2} \propto B_{0}^{-4}$. At a magnetic field of $15 \mathrm{G}$ where $q / c \approx 400$, the fraction of atoms in the $\left|m_{\mathrm{F}}=-1\right\rangle$ state is exceedingly small and thus the two-component approximation to the domain boundary should be quite accurate, as indicated by our data. At lower magnetic fields, the population in the $\left|m_{\mathrm{F}}=-1\right\rangle$ state plays an increasingly important role.

Guided by these simple approximate treatment, one can also explicitly calculate the spinor wavefunction at the domain boundary which minimizes the total energy. Such calculations were presented in Ref. [5], and the results are summarized in Figs. 32 and 33. One finds indeed that the fractional population of atoms in the $\left|m_{\mathrm{F}}=-1\right\rangle$ state is non-zero at all magnetic fields and scales as $B_{0}^{-4}$ at high magnetic fields. The introduction of $m_{F}=-1$ atoms in the boundary layer increases the penetration depth of $m_{F}=0$ atoms in the boundary region, and thus should increase the rate of tunneling across the domain boundary. The magnetic field at which this effect becomes significant, according to these calculations, is about $1 \mathrm{G}$ for our experimental conditions and agrees with the magnetic field value below which an increased tunneling rate was observed.

\footnotetext{
${ }^{5}$ Unlike in Ref. 97 , we do not apply here a one-dimensional approximation to reduce the effective interaction energy by averaging over the radial directions.

${ }^{6}$ In our paper, an incorrect solution was given which indicated a high-field scaling of $\epsilon^{2} \propto B_{0}^{-2} 99$.
} 

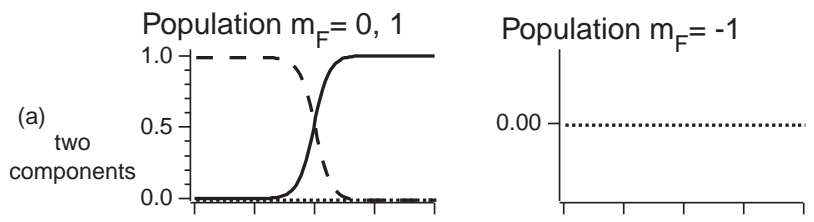

(b) $15 \mathrm{G}$
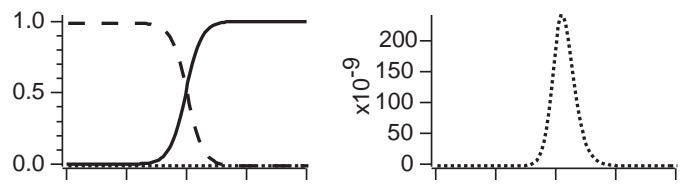

(c) $5 \mathrm{G}$
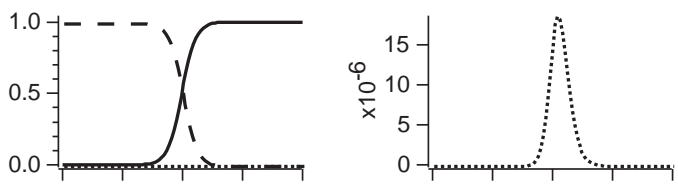

(d) $1 \mathrm{G}$
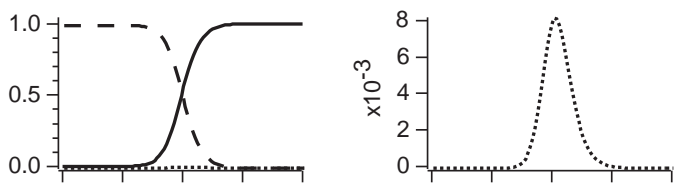

(e) $0.5 \mathrm{G}$
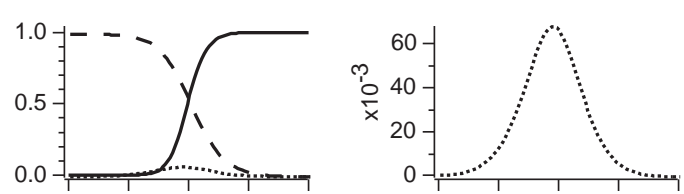

(f) $0.25 \mathrm{G}$
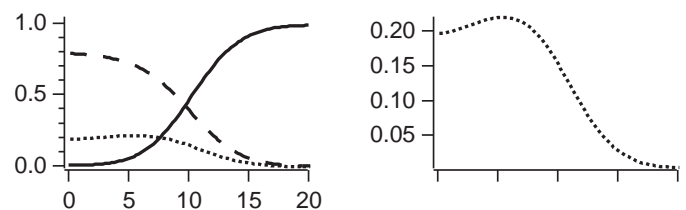

Fig. 32. Numerical calculation of the spin structure at the domain boundaries at different magnetic fields. Shown are the fractional population in the $\left|m_{\mathrm{F}}=1\right\rangle$ (dashed line), $\left|m_{\mathrm{F}}=0\right\rangle$ (solid line), and $\left|m_{\mathrm{F}}=-1\right\rangle$ (dotted line) states, with the $\left|m_{\mathrm{F}}=-1\right\rangle$ population shown at right on an expanded scale. In (a), the calculation was restricted to just the $m_{F}=0$ and $m_{F}=1$ components, and hence the results are magnetic field independent. In the remaining graphs, all three components were considered at magnetic fields of (b) $15 \mathrm{G}$, (c) $5 \mathrm{G}$, (d) $1 \mathrm{G}$, (e) $0.5 \mathrm{G}$, and (f) $0.25 \mathrm{G}$. As the magnetic field is decreased, the population in the $\left|m_{\mathrm{F}}=-1\right\rangle$ state increases, and the boundary region becomes wider. Further details are found in Ref. [5. 


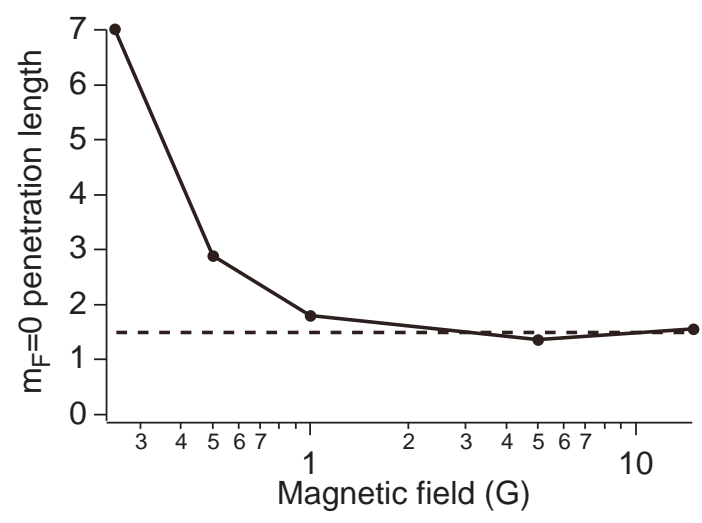

Fig. 33. Variation of the spin-domain boundary width with magnetic field. The calculated spatial variation of the fractional population of atoms in the $\left|m_{\mathrm{F}}=0\right\rangle$ state was fitted to the function $f(z)=\left[1-\operatorname{Erf}\left(\left(z-z_{0}\right) / \xi_{0}\right)\right] / 2$, where $\xi_{0}$ characterizes the penetration length of the $m_{F}=0$ component in the boundary region. The points correspond to graphs $(b-f)$ of Fig. 32, while the dashed line shows the penetration length calculated for the two-component case. The penetration length is close to the twocomponent limit at high magnetic fields, and then increases sharply at lower fields beginning at about $1 \mathrm{G}$, in agreement with our experimental observation of an increased tunneling rate below this magnetic field value.

\section{Conclusions}

These notes have reviewed advances in atomic BEC in two areas, spinor condensates and light scattering. Many further advances are described in other contributions to this volume. This rapid pace of developments during the last few years has taken the community by surprise. After decades of an elusive search nobody expected that condensates would be so robust and relatively easy to manipulate. Also, nobody imagined that such a simple system would pose so many challenges, not only to experimentalists, but also to our fundamental understanding of physics. The list of future challenges is long and includes the complete characterization of elastic and inelastic collisions at ultralow temperatures, the exploration of superfluidity, vortices, and second sound in Bose gases, the study of quantum-degenerate molecules and Fermi gases, the development of practical "high-power" atom lasers, and their application in atom optics and precision measurements.

\section{Acknowledgments}

We are grateful to Chris Westbrook and Robin Kaiser for organizing a stimulating summer school, to Shin Inouye for valuable discussions, to Chandra Raman and Ananth Chikkatur for comments on the manuscript. The BEC work at MIT was done in collaboration with M.R. Andrews, A.P. Chikkatur, K.B. Davis, D.S. Durfee, A. Görlitz, S. Gupta, Z. Hadzibabic, S. Inouye, M. Köhl, C.E. Kuklewicz, M.-O. Mewes, H.-J. Miesner, R. Onofrio, T. Pfau, D.E. Pritchard, C. Raman, D.M. Stamper-Kurn, J. Stenger, C.G. Townsend, N.J. van Druten, and J. Vogels. This work was supported by NSF, ONR, JSEP, ARO, NASA, and the David and Lucile Packard Foundation. D.M.S.-K. acknowledges additional support from JSEP, and is presently supported by a Millikan Prize Postdoctoral Fellowship.

\section{References}

[1] W. Ketterle, D.S. Durfee, and D.M. Stamper-Kurn, in Bose-Einstein condensation in atomic gases, Proceedings of the International School of Physics Enrico Fermi, Course CXL, edited by M. Inguscio, S. Stringari, and C.E. Wieman (IOS Press, Amsterdam, 1999), pp. 67-176.

[2] M. Inguscio, S. Stringari, and C.E. Wieman, Bose-Einstein condensation in atomic gases, Proceedings of the International School of Physics Enrico Fermi, Course CXL (IOS Press, Amsterdam, 1999), (editors).

[3] F. Dalfovo, S. Giorgini, L.P. Pitaevskii, and S. Stringari, Rev. Mod. Phys. 71, 463 (1999). 
[4] A.S. Parkins and D.F. Walls, Phys. Rep. 303, 1 (1998).

[5] D.M. Stamper-Kurn, Ph.D. thesis, Massachusetts Institute of Technology, 1999.

[6] D.M. Stamper-Kurn, A.P. Chikkatur, A. Görlitz, S. Gupta, S. Inouye, J. Stenger, D.E. Pritchard, and W. Ketterle, proceedings of the Tenth Conference on Many-Body Physics (MBX), Seattle, Sept. 1999, in print.

[7] J. Javanainen, Phys. Rev. Lett. 72, 2375 (1994).

[8] B.V. Svistunov and G.V. Shlyapnikov, Sov. Phys. JETP 71, 71 (1990).

[9] H.D. Politzer, Phys. Rev. A 43, 6444 (1991).

[10] J. Stenger, S. Inouye, A.P. Chikkatur, D.M. Stamper-Kurn, D.E. Pritchard, and W. Ketterle, Phys. Rev. Lett. 82, 4569 (1999).

[11] D.M. Stamper-Kurn, A.P. Chikkatur, A. Görlitz, S. Inouye, S. Gupta, D.E. Pritchard, and W. Ketterle, Phys. Rev. Lett. 83, 2876 (1999).

[12] P.L. Gould, G.A. Ruff, and D.E. Pritchard, Phys. Rev. Lett. 56, 827 (1986).

[13] P.J. Martin, B.G. Oldaker, A.H. Miklich, and D.E. Pritchard, Phys. Rev. Lett. 60, 515 (1988).

[14] Y.B. Ovchinnikov, J.H. Müller, M.R. Doery, E.J.D. Vredenbregt, K. Helmerson, S.L. Rolston, and W.D. Phillips, Phys. Rev. Lett. 83, 284 (1999).

[15] M. Kozuma, L. Deng, E.W. Hagley, J. Wen, R. Lutwak, K. Helmerson, S.L. Rolston, and W.D. Phillips, Phys. Rev. Lett. 82, 871 (1999).

[16] J.-Y. Courtois, G. Grynberg, B. Lounis, and P. Verkerk, Phys. Rev. Lett. 72, 3017 (1994).

[17] L. van Hove, Phys. Rev. 96, 249 (1954).

[18] T.J. Greytak, in Quantum Liquids, edited by J. Ruvalds and T. Regge (North-Holland, New York, 1978), pp. 121-165.

[19] P.E. Sokol, in Bose-Einstein Condensation, edited by A. Griffin, D.W. Snoke, and S. Stringari (Cambridge University Press, Cambridge, 1995), pp. 51-85.

[20] P. Nozières and D. Pines, The Theory of Quantum Liquids (Addison-Wesley, Redwood City, CA, 1990).

[21] A. Griffin, Excitations in a Bose-condensed liquid (Cambridge University Press, Cambridge, 1993).

[22] J. Javanainen, Phys. Rev. Lett. 75, 1927 (1995).

[23] J. Javanainen and J. Ruostekoski, Phys. Rev. A 52, 3033 (1995).

[24] R. Graham and D. Walls, Phys. Rev. Lett. 76, 1774 (1996).

[25] F. Zambelli, L. Pitaevskii, D.M. Stamper-Kurn, and S. Stringari, preprint cond-mat/9912089.

[26] C. Cohen-Tannoudji, J. Dupont-Roc, and G. Grynberg, Atom-Photon Interactions (Wiley, New York, 1992).

[27] N.N. Bogoliubov, J. Phys. (USSR) 11, 23 (1947).

[28] P.J. Price, Phys. Rev. 94, 257 (1954).

[29] K. Huang, Statistical Mechanics (Wiley, New York, 1987).

[30] E.P. Gross, Nuovo Cimento 20, 454 (1961).

[31] L.P. Pitaevskii, Sov. Phys. JETP 13, 451 (1961).

[32] S. Inouye, A.P. Chikkatur, D.M. Stamper-Kurn, J. Stenger, D.E. Pritchard, and W. Ketterle, Science 285, 571 (1999).

[33] F. Dalfovo, S. Giorgini, M. Guilleumas, L.P. Pitaevskii, and S. Stringari, Phys. Rev. A 56, 3840 (1997).

[34] T.C. Killian, preprint, physics/9908002.

[35] T.C. Killian, D.G. Fried, L. Willmann, D. Landhuis, S.C. Moss, T.J. Greytak, and D. Kleppner, Phys. Rev. Lett. 81, 3907 (1998).

[36] D.G. Fried, T.C. Killian, L. Willmann, D. Landhuis, S.C. Moss, D. Kleppner, and T.J. Greytak, Phys. Rev. Lett. 81, 3811 (1998).

[37] M.O. Mewes, M.R. Andrews, N.J. Van Druten, D.M. Kurn, D.S. Durfee, and W. Ketterle, Phys. Rev. Lett. 77, 416 (1996).

[38] E.W. Hagley, L. Deng, M. Kozuma, M. Trippenbach, Y.B. Band, M. Edwards, M. Doery, P.S. Julienne, K. Helmerson, S.L. Rolston, and W.D. Phillips, Phys. Rev. Lett. 83, 3112 (1999).

[39] L. Deng, E.W. Hagley, J. Wen, M. Trippenbach, Y. Band, P.S. Julienne, J.E. Simsarian, K. Helmerson, S.L. Rolston, and W.D. Phillips, Nature 398, 218 (1999).

[40] Y. Castin and R. Dum, Phys. Rev. Lett. 77, 5315 (1996).

[41] M.R. Andrews, C.G. Townsend, H.-J. Miesner, D.S. Durfee, D.M. Kurn, and W. Ketterle, Science 275, 637 (1997).

[42] I. Bloch, T.W. Hänsch, and T. Esslinger, Nature 403, 166 (2000).

[43] A.B. Kuklov and B.V. Svistunov, Phys. Rev. A 60, R769 (1999).

[44] D.M. Stamper-Kurn, M.R. Andrews, A.P. Chikkatur, S. Inouye, H.-J. Miesner, J. Stenger, and W. Ketterle, Phys. Rev. Lett. 80, 2027 (1998).

[45] S. Inouye, M.R. Andrews, J. Stenger, H.-J. Miesner, D.M. Stamper-Kurn, and W. Ketterle, Nature 392, 151 (1998).

[46] H. Saito and M. Ueda, Phys. Rev. A 60, 3990 (1999).

[47] M.G. Moore and P. Meystre, Phys. Rev. A 59, 1754 (1999). 
[48] N.E. Rehler and J.H. Eberly, Phys. Rev. A 3, 1735 (1971).

[49] R.H. Dicke, in Quantum Electronics, Proceedings of the third international congress, edited by P. Grivet and N. Bloembergen (Columbia University Press, New York, 1964), p. 35.

[50] C.K. Law and N.P. Bigelow, Phys. Rev. A 58, 4791 (1998).

[51] L. Moi, P. Goy, M. Gross, J.M. Raimond, C. Fabre, and S. Haroche, Phys. Rev. A 27, 2043 (1983).

[52] R. Wynar, R.S. Freeland, D.J. Han, C. Ryu, and D.J. Heinzen, Science 287, 1016 (2000).

[53] F.A. van Abelen and B.J. Verhaar, Phys. Rev. Lett. 83, 1550 (1999).

[54] Q.H.F. Vrehen and H.M. Gibbs, in Topics in Current Physics, Vol. 27 of Dissipative System in Quantum Optics, edited by R. Bonifacio (Springer, Heidelberg, 1982), pp. 111-147.

[55] N. Skribanowitz, I.P. Herman, J.C. MacGillivray, and M.S. Feld, Phys. Rev. Lett. 30, 309 (1973).

[56] R. Bonifacio and L.A. Lugiato, Phys. Rev. A 11, 1507 (1975).

[57] M.F.H. Shuurmanns, Q.H.F. Vrehen, D. Polder, and H.M. Gibbs, Adv. At. Mol. Phys. 17, 167 (1981).

[58] M. Gross and S. Haroche, Phys. Rep. 93, 301 (1982).

[59] R.H. Dicke, Phys. Rev. 93, 99 (1954).

[60] R. Bonifacio and L. De Salvo, Nuclear Instruments and Methods in Physics Research A 341, 360 (1994).

[61] M.G. Moore and P. Meystre, Phys. Rev. A 58, 3248 (1998).

[62] P.R. Berman, Phys. Rev. A 59, 585 (1999).

[63] M. Olshanii, Y. Castin, and J. Dalibard, in Laser Spectroscopy XII, edited by M. Inguscio, M. Allegrini, and A. Sasso (World Scientific, Singapore, 1996), pp. 7-12.

[64] H.M. Wiseman and M.J. Collett, Physics Letters A 202, 246 (1995).

[65] U. Janicke and M. Wilkens, in Ultracold Atoms and Bose-Einstein-Condensation, 1996, Vol. 7 of OSA Trends in Optics and Photonics Series, Vol. 7, edited by K. Burnett (Optical Society of America, Washington D.C., 1996), pp. $38-47$.

[66] R.Y. Chiao, C.H. Townes, and B.P. Stoicheff, Phys. Rev. Lett. 12, 592 (1964).

[67] M.C. Cross and P.C. Hohenberg, Rev. Mod. Phys. 65, 851 (1993).

[68] S. Inouye, T. Pfau, S. Gupta, A.P. Chikkatur, A. Görlitz, D.E. Pritchard, and W. Ketterle, Nature 402,641 (1999).

[69] M. Kozuma, Y. Suzuki, Y. Torii, T. Sugiura, T. Kuga, E.W. Hagley, and L. Deng, Science 286, 2309 (1999).

[70] J. Schmiedmayer, M.S. Chapman, C.R. Ekstrom, T.D. Hammond, S. Wehinger, and D.E. Pritchard, Phys. Rev. Lett. 74, 1043 (1995).

[71] G.E. Stedman, Rep. Prog. Phys. 60, 615 (1997).

[72] T.L. Gustavson, P. Bouyer, and M.A. Kasevich, Phys. Rev. Lett. 78, 2046 (1997).

[73] L. Guttman and J.R. Arnold, Phys. Rev. 92, 547 (1953).

[74] I.M. Khalatnikov, Soviet Physics JEPT 5, 542 (1957).

[75] W.B. Colson and A.L. Fetter, J. Low Temp. Phys. 33, 231 (1978).

[76] E.D. Siggia and E.A. Ruckenstein, Phys. Rev. Lett. 44, 1423 (1980).

[77] T.-L. Ho and V.B. Shenoy, Phys. Rev. Lett. 77, 3276 (1996).

[78] B.D. Esry, C.H. Greene, J.P.J. Burke, and J.L. Bohn, Phys. Rev. Lett. 78, 3594 (1997).

[79] E.V. Goldstein and P. Meystre, Phys. Rev. A 55, 2935 (1997).

[80] C.K. Law, H. Pu, N.P. Bigelow, and J.H. Eberly, Phys. Rev. Lett. 79, 3105 (1997).

[81] C.J. Myatt, E.A. Burt, R.W. Ghrist, E.A. Cornell, and C.E. Wieman, Phys. Rev. Lett. 78, 586 (1997).

[82] J.P.J. Burke, J.L. Bohn, B.D. Esry, and C.H. Greene, Phys. Rev. A 55, R2511 (1997).

[83] S.J.J.M.F. Kokkelmans, H.M.J.M. Boesten, and B.J. Verhaar, Phys. Rev. A 55, R1589 (1997).

[84] P.S. Julienne, F.H. Mies, E. Tiesinga, and C.J. Williams, Phys. Rev. Lett. 78, 1880 (1997).

[85] D.S. Hall, M.R. Matthews, J.R. Ensher, C.E. Wieman, and E.A. Cornell, Phys. Rev. Lett. 81, 4531 (1998).

[86] D.S. Hall, M.R. Matthews, C.E. Wieman, and E.A. Cornell, Phys. Rev. Lett. 81, 1543 (1998).

[87] J. Williams, R. Walser, J. Cooper, E. Cornell, and M. Holland, Phys. Rev. A 59, R31 (1999).

[88] M.R. Matthews, B.P. Anderson, P.C. Haljan, D.S. Hall, M.J. Holland, J.E. Williams, C.E. Wieman, and E.A. Cornell, Phys. Rev. Lett. 83, 3358 (1999).

[89] J. Williams, R. Walser, J. Cooper, E.A. Cornell, and M. Holland, Phys. Rev. A 61, March (2000).

[90] M.R. Matthews, B.P. Anderson, P.C. Haljan, D.S. Hall, C.E. Wieman, and E.A. Cornell, Phys. Rev. Lett. 83, 2498 (1999).

[91] J.E. Williams and M.J. Holland, Nature 401, 568 (1999).

[92] E.A. Cornell, D.S. Hall, M.R. Matthews, and C.E. Wieman, J. Low Temp. Phys. 113, 151 (1998).

[93] E.A. Cornell, J.R. Ensher, and C.E. Wieman, in Bose-Einstein condensation in atomic gases, Proceedings of the International School of Physics Enrico Fermi, Course CXL, edited by M. Inguscio, S. Stringari, and C.E. Wieman (IOS Press, Amsterdam, 1999), pp. 15-66.

[94] T.-L. Ho, Phys. Rev. Lett. 81, 742 (1998). 
[95] T. Ohmi and K. Machida, J. Phys. Soc. Jap. 67, 1822 (1998).

[96] C.K. Law, H. Pu, and N.P. Bigelow, Phys. Rev. Lett. 81, 5257 (1998).

[97] J. Stenger, S. Inouye, D.M. Stamper-Kurn, H.-J. Miesner, A.P. Chikkatur, and W. Ketterle, Nature 396, 345 (1998).

[98] H.-J. Miesner, D.M. Stamper-Kurn, J. Stenger, S. Inouye, A.P. Chikkatur, and W. Ketterle, Phys. Rev. Lett. 82, 2228 (1999).

[99] D.M. Stamper-Kurn, H.-J. Miesner, A.P. Chikkatur, S. Inouye, J. Stenger, and W. Ketterle, Phys. Rev. Lett. 83, 661 (1999).

[100] J. Stenger, D.M. Stamper-Kurn, M.R. Andrews, A.P. Chikkatur, S. Inouye, H.-J. Miesner, and W. Ketterle, J. Low Temp. Phys. 113, 167 (1998).

[101] W.-J. Huang and S.-C. Gou, Phys. Rev. A 59, 4608 (1999).

[102] M. Koashi and M. Ueda, Phys. Rev. Lett. 84, 1066 (2000).

[103] T.-L. Ho and S.K. Yip, preprint cond-mat/9905339.

[104] E. Tiesinga, C.J. Williams, P.S. Julienne, K.M. Jones, P.D. Lett, and W.D. Phillips, J. Res. Natl. Inst. Stand. Technol. 101, 505 (1996).

[105] J.P. J. Burke, C.H. Greene, and J.L. Bohn, Phys. Rev. Lett. 81, 3355 (1998).

[106] M.-O. Mewes, M.R. Andrews, D.M. Kurn, D.S. Durfee, C.G. Townsend, and W. Ketterle, Phys. Rev. Lett. 78, $582(1997)$.

[107] W.-J. Huang and S.-C. Gou, preprint, cond-mat/9905435.

[108] T. Isoshima, K. Machida, and T. Ohmi, Phys. Rev. A 60, 4857 (1999).

[109] P. Ao and S.T. Chui, Phys. Rev. A 58, 4836 (1998).

[110] H. Pu and N.P. Bigelow, Phys. Rev. Lett. 80, 1130 (1998).

[111] S.T. Chui and P. Ao, preprint, cond-mat/9902275.

[112] H. Pu, C.K. Law, S. Raghavan, J.H. Eberly, and N.P. Bigelow, Phys. Rev. A 60, 1463 (1999).

[113] B.D. Josephson, Phys. Lett. 1, 251 (1962).

[114] J. Javanainen, Phys. Rev. Lett. 57, 3164 (1986).

[115] A. Smerzi, S. Fantoni, S. Giovanazzi, and S.R. Shenoy, Phys. Rev. Lett. 79, 4950 (1997).

[116] I. Zapata, F. Sols, and A.J. Leggett, Phys. Rev. A 57, R28 (1998).

[117] B.P. Anderson and M.A. Kasevich, Science 282, 1686 (1998).

[118] R.H. Fowler and L. Nordheim, Proc. Roy. Soc. London, Ser. A 119, 173 (1928). 